\title{
Miocene deep-water agglutinated foraminifera from ODP Hole 909c: Implications for the paleoceanography of the Fram Strait Area, Greenland Sea
}

\author{
Michael A. Kaminski ${ }^{1}$, Lóránd Silye ${ }^{2}$ and Severyn Kender ${ }^{3}$ \\ ${ }^{1}$ Department of Earth Sciences, University College London, Gower Street, London WC1E 6BT, U.K. \\ email: m.kaminski@ucl.ac.uk \\ 2Department of Geology, Babes-Bolyai University, Str. Kogalniceanu 1, 400084, Cluj-Napoca, Romania \\ ${ }^{3}$ Department of Earth Sciences, University College London, Gower Street, London WC1E 6BT, U.K. \\ email: s.kender@ucl.ac.uk
}

\begin{abstract}
Deep-water agglutinated Foraminifera (DWAF) are investigated from Miocene sediments recovered from ODP Hole 909C in the Fram Strait, Norwegian-Greenland Sea. We studied 125 samples from Cores 909C-50R to -103R, and recovered over 60 species of DWAF. The faunal succession in Hole $909 \mathrm{C}$ is subdivided into three assemblages based on the stratigraphic ranges of characteristic cosmopolitan taxa. These are: (1) a diverse Reticulophragmium amplectens - Reophanus berggreni Assemblage in Cores 909C-100R-2 to -91R-1 (1040.71-952.78mbsf); (2) a Reticulophragmium amplectens Assemblage in Cores 909C-87R-2, to -71R-3 (915.7-762.68mbsf); and (3) a low-diversity Reticulophragmium rotundidorsatum Assemblage in Cores 909C-71R-1 to -55R-1 (759.68-605.52mbsf). The DWAF assemblages are correlated to the standard chronostratigraphy using dinoflagellate cysts and magnetostratigraphy. The stratigraphic ranges of some well-known Paleogene DWAF species extend far into the Miocene at this locality, confirming the hypothesis that the Arctic and northern Norwegian Sea basins served as a refuge for these species long after they disappeared from the North Atlantic stratigraphic record. The taxonomic affinities of the Miocene assemblages from Hole 909C supports the idea that an estuarine circulation pattern has been in place between the Arctic Ocean and Greenland Sea basins since at least the early Miocene. Changes in the benthic foraminiferal morphogroups within the $R$. rotundidorsatum Assemblage correlate with an increase in total organic carbon, indicating an increase in oceanic productivity in the Fram Strait region during the late Miocene.
\end{abstract}

\section{INTRODUCTION}

Deep-water agglutinated foraminifera, commonly known by the acronym DWAF, represent one of the most useful groups of microfossils for stratigraphical and paleoenvironmental interpretations of high-latitude Cretaceous to Miocene deep-water sedimentary sequences. They are often the only benthic microfossils preserved in the deep-water deposits of the Boreal seas. Key studies carried out to date on the DWAF of the northern North Atlantic region almost exclusively dealt with the taxonomy and biostratigraphy of Paleogene taxa (Gradstein and Berggren 1981; Miller et al. 1982; Verdenius and van Hinte 1983; Gradstein and Kaminski 1989, 1997; Gradstein et al. 1994, Kaminski et al. 1990; Kaminski and Geroch 1997; Nagy et al. 1997, 2000, 2004; Kaminski and Austin 1999). The fact that so few studies have been carried out on the Neogene DWAF from the North Atlantic is a consequence of either the lack of Miocene recovery and/or well-preserved Miocene agglutinated foraminiferal assemblages from most drilled locations. Miocene sediments from the Central Norwegian Sea are often biosiliceous, and therefore unfavourable for DWAF preservation. An exception to this was the discovery of well preserved and anomalously young Miocene DWAF in ODP Hole 909C drilled in the Fram Strait area of the Greenland Sea, published in the scientific results of the ODP Leg 151 "North Atlantic-Arctic Gateways" expedition (Osterman and Spiegler 1996).

The Fram Strait is a critical ocean "gateway" - it is the only conduit to exchange deep waters between the Arctic and the At- lantic Oceans. One of the primary objectives of drilling at Site 909 was to document the timing of the opening of a deep passageway through the Fram Strait, and the history of deep and shallow water exchange between the Arctic and the world ocean (Thiede and Myhre 1996). The DWAF of ODP Hole 909C therefore play a crucial role for our understanding of faunal and water mass exchange between the Norwegian-Greenland Sea and the Arctic Ocean. Moreover, this fauna may be useful for biostratigraphical correlation between the North Atlantic and the Arctic IODP holes. The proposed ages for the opening of the Fram Strait gateway span from the early Oligocene, Anomaly 13, to the late Miocene, Anomaly 5 (Thiede and Myhre 1996). However, the Fram Strait has a strongly oblique opening and is in the proximity of the submerged Hovgard Microcontinent, which may have acted as a plug until the Miocene (Myhre and Thiede 1995, Thiede and Myhre 1996). This makes it more difficult to estimate the timing of the opening of the Fram Strait and the initiation of deep-water connections between the Norwegian-Greenland Sea and Artic Ocean.

The main objectives of this study are to (1) revise the taxonomy of the benthic foraminifera recovered from ODP Hole 909C; (2) provide a continuous biostratigraphic and proxy record for sea-floor conditions during the Miocene; (3) establish the sequence of benthic foraminiferal assemblages that will contribute to our understanding of Artic biota from the Eocene to late Miocene; (4) test whether agglutinated foraminifera can be used to interpret the history of deep water connections between Norwegian Sea and Arctic Ocean through the Fram Strait; and (5) to 
better understand the paleoproductivity and the timing and onset of deep convection in the Fram Strait region.

Toward these aims, the agglutinated foraminiferal record from Hole 909C in the Fram Strait is particularly important to test the hypothesis that the Arctic may have served as a refuge, for certain species as previously suggested (Kaminski et al. 1990, Osterman and Spiegler 1996). This hypothesis could explain why typical agglutinated Paleogene species have been found in younger sediments in the Arctic. We wish to assess to what degree faunas in the Fram Strait area are endemic, and whether they contain any typical Arctic species. However, these latter questions can only be fully answered after the scientific results of IODP drilling in the Arctic Ocean are published.

\section{BACKGROUND}

\section{Location}

ODP Site 909 is located in the southern area of the Fram Strait near the Greenland-Spitsbergen sill terrace immediately north of the Hovgård Ridge (Myhre and Thiede 1995). This gently elevated area is protected against the influx of turbidites from the Svalbard and Greenland margins (Shipboard Scientific Party 1995). Hole $909 \mathrm{C}$ was drilled on a small abyssal terrace of the Fram Strait, at $78^{\circ} 35.096^{\prime} \mathrm{N}, 3^{\circ} 4.222^{\prime} \mathrm{E}$ (text-fig. 1), at a water depth of $2518 \mathrm{~m}$ (drill pipe measurement from sea level), with a penetration of $1061.8 \mathrm{mbsf}$. The total length of cored section was $976.8 \mathrm{~m}$, with an average core recovery of $61.9 \%$ (Shipboard Scientific Party 1995).

\section{Lithology}

The sediments recovered are dominated by silty clay throughout the entire cored section. Clay, clayey silt, clayey mud, and silty mud are also common. Calcareous clays and carbonate-bearing silty clays, containing thin layers and nodules of inorganic carbonates are characteristic, and are present in small quantities throughout the sedimentary sequence. However, biogenic sediments are present only in specific intervals as trace components. Dropstones occur only in the interval of 85.8$314.26 \mathrm{mbsf}$, dated to the early Pliocene (4.6 Ma.) to Recent by means of the magnetic polarity record (Shipboard Scientific Party 1995).

Three lithologic units were recognized in Hole 909C (Shipboard Scientific Party 1995). The agglutinated foraminiferal assemblages described here appear within Unit III (518.3$1061.8 \mathrm{mbsf}$ ), which is subdivided into Lithologic Subunit IIIA (518.3-923.4 mbsf) and Lithologic Subunit IIIB (923.4mbsf$1061.8 \mathrm{mbsf}$ ). These subunits were correlated to the Miocene, based on magnetostratigraphy and the occurrence of latest Oligocene/earliest Miocene calcareous nannofossil assemblage at the base of the hole, and the continuous occurrence of Miocene dinoflagellate cysts below Core 23R (Hull, et al. 1996; O'Connell et al. 1996; Poulsen et al. 1996).

The sediments of Subunit IIIA (Cores -46R-1 through $-87 \mathrm{R}-\mathrm{CC}$ ) are dominated by very dark gray silty clay, with coarser grains at the base. The top of the subunit is placed at $518.3 \mathrm{mbsf}$, defined by the presence of laminations below this horizon. Clayey silt, silty mud, several meters-thick intervals of carbonate, and carbonate-bearing sediments (concretions) are also present as minor constituents. Lamination is a characteristic feature of this subunit. Very dark gray laminae are generally interlayered with dark grayish brown, grayish brown, and light brownish gray laminae. Bioturbation is observed throughout al- most the entire section. Even within the best-developed laminae rare burrows can be observed. Many originally laminated intervals are extensively bioturbated, and the burrows parallel to bedding plane appear to enhance rather than obscure the laminations. The thin laminations of the Subunit IIIA were possibly caused by rhythmic depositional processes, extending over 15 million years (Shipboard Scientific Party 1995).

The sediments of Subunit IIIB (Cores -88R-1 through $-103 R-C C$ ) are distinguished from the overlying sediments by the presence of slump structures. The slumps exhibit sharp basal contacts and millimeter- to tens of meter-scale folds, rip-up clasts, tilted and pinched layers. Sometimes the disturbed layers have sharp upper contacts. The slumps are interpreted as results of rapid sediment accumulation and small textural differences. Bioturbation is generally moderate to extensive, but is absent from the thickest slumps. The most important sediments of the subunit include grayish brown, olive gray, dark greenish gray, dark gray colored silty clay, clayey silt, mud, and silty mud. Agglutinated and rare calcareous benthic foraminifera are present in the sediments of Subunit IIIB. The calcareous foraminifera are usually decalcified, and many of the agglutinated specimens are fragmented (Shipboard Scientific Party 1995).

\section{Biostratigraphy}

The age control of Hole $909 \mathrm{C}$ is accomplished by means of planktonic foraminifera, calcareous nannofossils, and palynomorphs (Spiegler 1996; Hull et al. 1996; Poulsen et al. 1996). Magnetic polarity records have also been used (O'Connell et al. 1996). The chronostratigraphy of the studied interval is summarized in text-figure 2 .

The oldest sediments recovered from Hole 909C are regarded to be earliest Miocene based on the occurrence of the calcareous nannofossil Helicosphaera carteri in Sections 909C-101R-2 to 102R-CC (Shipboard Scientific Party 1995). Dinocysts also support an early Miocene (Aquitanian?-Langhian?) age based on the occurrence of Hystriochokolpoma sp. 2 in Sample 909C-103R-CC (Poulsen et al. 1996). This species was not recorded below the Miocene at Site 643 (Manum et al. 1989).

Middle Miocene (Langhian-Serravallian) sediments occur in dinocyst Zone Mio2 (Langhian), Mio3 (Langhian-Serravallian) and Mio4 (Serravallian). Zone Mio2 is defined between the FO (first occurrence) of Labyrinthodinium truncatum in Sample 909C-98R-CC (1020.0mbsf), and the LO (last occurrence) of Apteodinium spiridoides identified in Sample 909C-95R-CC (992.0mbsf). Zone Mio3 is present up to the LOs of Apteodinium australiense and Distatodinium paradoxum in Samples 909C-87R-CC (916.23mbsf) and 81R-CC (861.78 mbsf), respectively. However, based on the FO of Achomosphera? andalousiensis in Sample 909C-82R-CC, the upper boundary of this dinocyst zone is placed at the FO of Achomosphera? andalousiensis, and not at the LO of Distatodinium paradoxum. The lower boundary of Zone Mio4 is placed at the FO of Achomosphera? andalousiensis in Sample 909C-82R-CC (867.8mbsf), whilst the top of the zone is defined by the LO of Systematophora placacantha in Sample 909C-70R-CC (Poulsen et al. 1996).

A Late Miocene (Tortonian) age is assigned to Zone Mio5, the upper boundary of which is defined by the LO of Paleocystodinium golzowense in Sample 909C-51R-CC, at 573.01mbsf (Poulsen et al. 1996). From 345.3 to $566.6 \mathrm{mbsf}$ (Samples 909C-27R-CC to 50R-CC) Upper Miocene to lower 


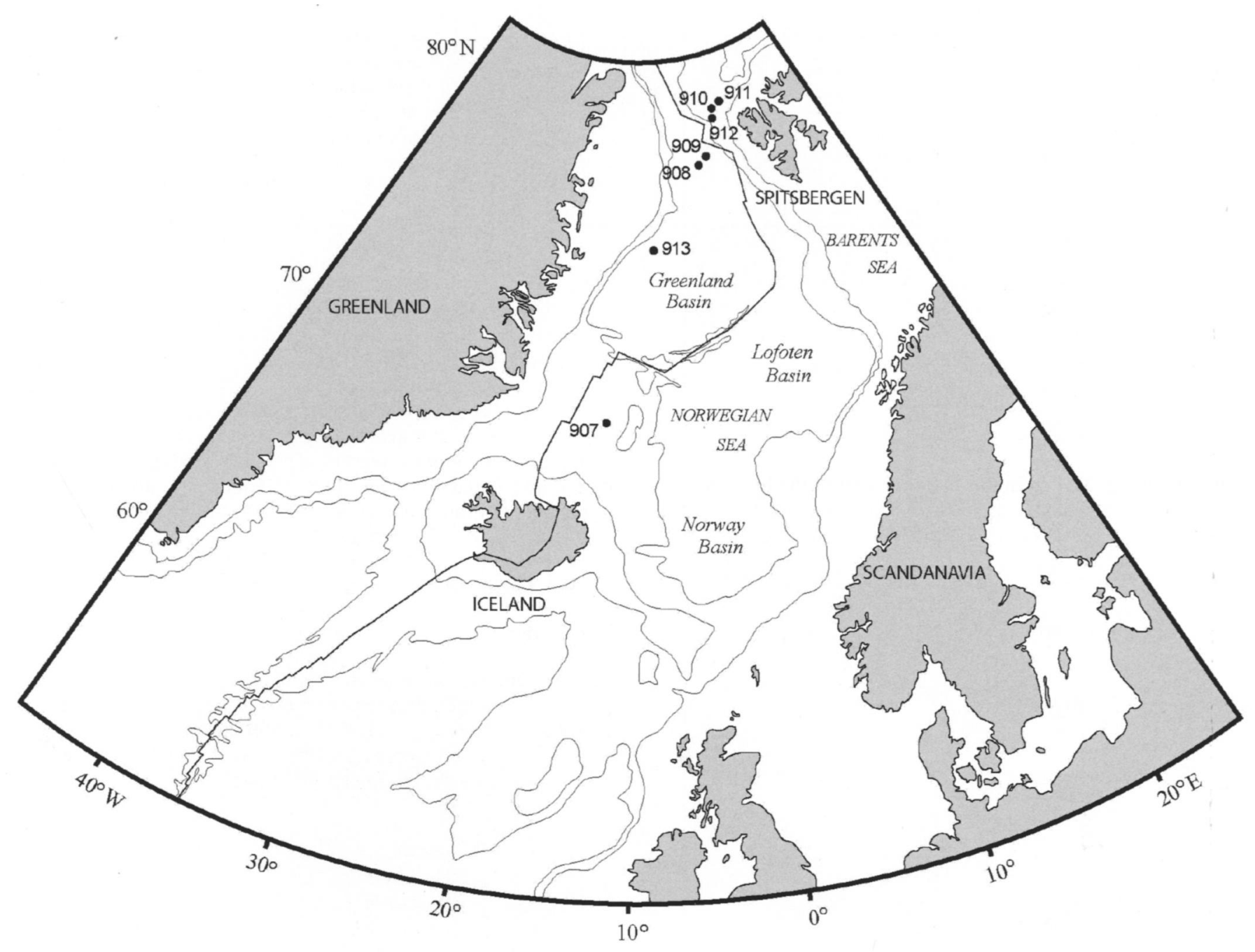

TEXT-FIGURE 1

Location of ODP Leg 151 Sites 907-913 (modified after Thiede and Myhre 1996).

Pliocene sediments have been reported (Hull et al. 1996; Poulsen et al. 1996).

Planktonic foraminifera in Hole 909C were studied by Spiegler (1996). According to these data, planktonic foraminifera are very rare in Hole 909C, owing to dissolution. Below Core 909C-43R, only about $10 \%$ of samples contained planktonic foraminifera. In Sample 909C-76R-1, 96-98cm (808.56mbsf) the FO of Globigerina bulloides indicates a late Miocene age, whilst in Sample 909C-89R-1, 97-100cm (934.07mbsf) the FO of Orbulina universa indicates a Middle Miocene or younger age (Spiegler 1996). No planktonic foraminifera were recovered in this study.

The agglutinated foraminifera from Hole 909C were initially studied by Osterman and Spiegler (1996). Agglutinated foraminifera were found to occur more or less continuously from the base of the hole up to Core 909C-52R,CC, and four assemblages are recognised. From the oldest to youngest these are:

(1) A lower to middle Miocene Rhabdammina/Reophax assemblage (Samples 151-909C-100R-CC to -103R-CC; 1048.4 to $1062.8 \mathrm{mbsf})$.
(2) A middle Miocene Reticulophragmium ex gr. rotundidorsatum and $R$. amplectens assemblage (Samples 151-909C90R-CC to -100R-1, $95-99 \mathrm{~cm} ; 952.4$ to $1039.75 \mathrm{mbsf}$ ).

(3) A middle Miocene Reticulophragmium amplectens assemblage (Samples 151-909C-71R-CC, $95-99 \mathrm{~cm}$, to $-87 \mathrm{R}-\mathrm{CC}$; 760.25 to $923.4 \mathrm{mbsf}$ ).

(4) A middle to upper Miocene Reticulophragmium ex gr. rotundidorsatum assemblage (Samples 151-909C-51R-1, 76$78 \mathrm{~cm}$, to $-70 \mathrm{R}-\mathrm{CC}$; 605.86 to $759.3 \mathrm{mbsf}$ ).

Osterman and Speigler (1996) noted that Reticulophragmium amplectens is normally an Eocene species that had not been previously reported in sediments of Miocene age. These authors excluded the possibility of reworking because (1) the site is located on a submarine high, and substantial tectonic uplift would be required to allow the erosion of Eocene deep water deposits and subsequent redeposition into Miocene sediments. Such a situation has not been confirmed. (2) None of the other microfossil groups recovered in this interval show signs of reworking, and all indicate a Miocene age (Osterman and Spiegler 1996; Hull et al. 1996). Additional foraminiferal taxa known 
M. A. Kaminski et al.: Miocene foraminifera from ODP Hole 909c: Paleoceanography of the Fram Strait Area, Greenland Sea

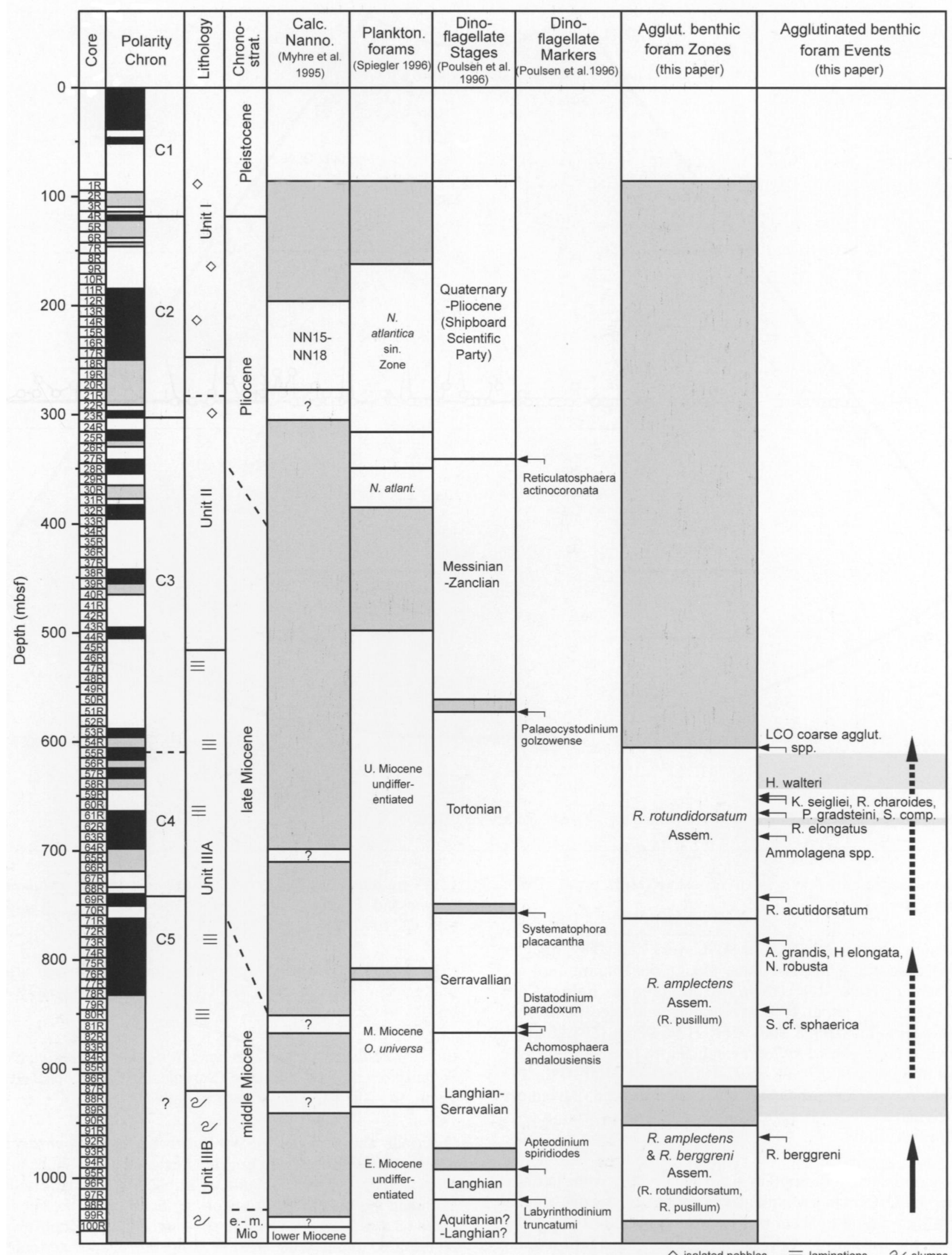

TEXT-FIGURE 2

Chronostratigraphy of Hole 909C, based on magnetostratigraphy (Shipboard Scientific Party 1995), palynology (after Poulsen et al. 1996), planktonic foraminifera (after Spiegler 1996) and calcareous nannofossils (after Hull et al. 1996). Also shown are our agglutinated foraminiferal assemblages and important last occurrences of DWAF. Arrows represent diversity/abundance (solid increasing, dashed decreasing). 

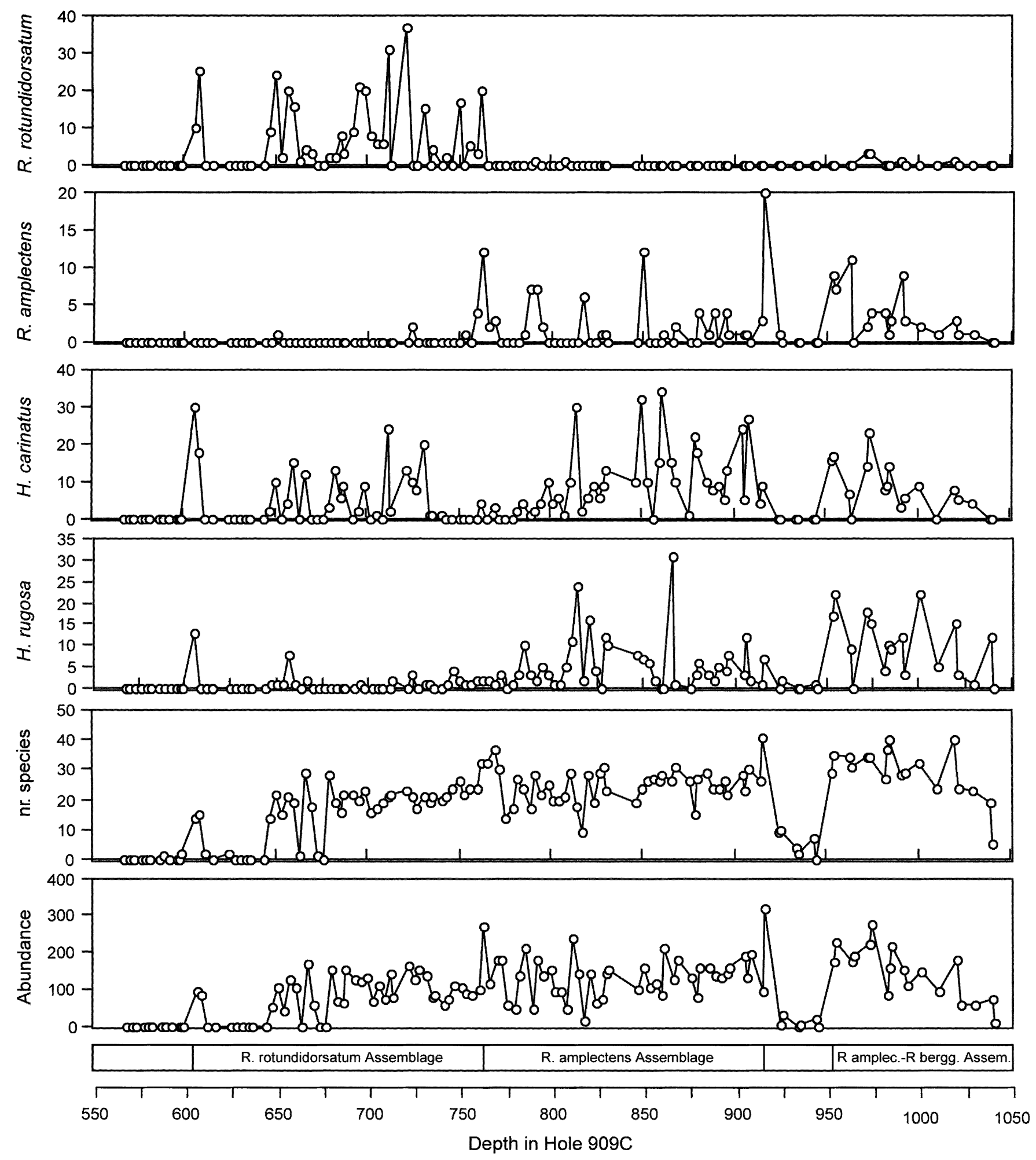

TEXT-FIGURE 3

Agglutinated foraminifera abundance and species number in Hole 909C (566.97 to $1040.71 \mathrm{mbsf}$ ). Also shown are the proportions of some abundant and biostratigraphically important taxa (Hyperammina rugosa, Haplophragmoides carinatus, $R$. amplectens. $R$. rotundidorsatum). 
from Paleogene strata in the Norwegian Sea - North Sea region were also reported.

\section{MATERIALS AND METHODS}

The standard methods of sample processing were applied to 125 samples collected from Hole 909C, from 566.97 to 1040.71 mbsf (Cores 909C-51R-1, 37-39cm through 100R-2, 41$42.5 \mathrm{~cm}$, except Core 151-909C-79R). The average volume of samples was $20 \mathrm{cc}$, and the number of samples collected per core was 2 or 3 . Each sample was dried overnight at about $40-50^{\circ} \mathrm{C}$ and then boiled in water with sodium carbonate $\left(\mathrm{Na}_{2} \mathrm{CO}_{3}\right)$ until completely disaggregated (about 15-20 minutes). The disaggregated samples were washed over a $63-\mu \mathrm{m}$ sieve. The residues were retained and dried at about $40-50^{\circ} \mathrm{C}$. Foraminifera were picked from the entire $>63-\mu \mathrm{m}$ residue. The specimens were photographed using a Zeiss 940 digital scanning electron microscope at Department of Earth Sciences, University College London.

\section{RESULTS}

\section{DWAF Assemblages}

The examination of samples from Hole 909C yielded 85 foraminiferal species and generic groupings (Appendix 1). The specimens throughout the studied interval are generally uncrushed and well preserved. Abundance and diversity display maximum values in the basal part of the studied interval (text-figure 3), and both parameters display minima within the slumped interval represented by Samples 909C-90R-2, 40-41.5cm to -88R-1, $39.5-41 \mathrm{~cm}$. Above the slumped interval, abundance and diversity are variable, but no meaningful trends are observed until $679 \mathrm{~m}$. Above this interval preservation was patchy, and both parameters decline upcore.

The composition of DWAF in our samples displays strong similarities to the assemblages described by Osterman and Spiegler (1996). Based on the ranges of distinctive taxa, we suggest the following subdivision into three assemblages (from oldest to youngest), here modified from Osterman and Spiegler (1996):

(1) Reticulophragmium amplectens - Reophanus berggreni Assemblage (Cores -100R-2, $41-42.5 \mathrm{~cm}$ to $-91 \mathrm{R}-1,38.5-40.5 \mathrm{~cm}$; 1040.7 to $952.8 \mathrm{mbsf}$ ) is characterized by the co-occurrence of Reticulophragmium rotundidorsatum (Hantken 1875), Reticulophragmium amplectens (Grzybowski 1898), Reticulophragmium pusillum (Brady 1881), and Reophanus berggreni Gradstein and Kaminski 1997. Additionally, Recurvoides spp., Psamminopelta gradsteini (Kaminski and Geroch 1997), Nothia robusta (Grzybowski 1898), Nothia latissima (Grzybowski 1898), and Hyperammina rugosa Verdenius and Van Hinte 1983 are common in this interval. This assemblage has the highest faunal density and diversity observed in Hole 909C (text-fig. 3).

Although the foraminiferal assemblage is similar to that described by Osterman and Spiegler (1996) from the same interval, we prefer not to use their nomenclature for this assemblage (Reticulophragmium ex gr. rotundidorsatum $-R$. amplectens Assemblage), to avoid confusion. Moreover, the distinctive species Reophanus berggreni Gradstein and Kaminski 1997 is present only in this interval in Hole 909C (the species was probably lumped under Hormosinella carpenteri by Osterman and Spiegler (1996)).
(2) Reticulophragmium amplectens Assemblage (Cores 909C$87 \mathrm{R}-2,40-42 \mathrm{~cm}$ to $-71 \mathrm{R}-3,38-39.5 \mathrm{~cm}$; 915.7 to $762.7 \mathrm{mbsf}$ ) is found above a slumped interval (Samples 909C-90R-2, $40-41.5 \mathrm{~cm}$ to $-88 \mathrm{R}-1,39.5-41 \mathrm{~cm})$. A rapid change in foraminiferal abundance (the lowest faunal abundances in Hole 909C) is observed in this interval (text-figure 3).

This assemblage is characterized by the presence of Reticulophragmium amplectens (Grzybowski 1898), R. pussilum (Brady 1881 ), the absence of $R$. rotundidorsatum (Hantken 1875), and the LOs of Nothia robusta (Grzybowski 1898), Aschemocella grandis (Grzybowski 1898), Hyperammina elongata Brady 1878 and Saccammina cf. sphaerica Brady 1879.

(3) Reticulophragmium rotundidorsatum Assemblage (Cores 909C-71R-1, 38.5-40m to $55 \mathrm{R}-1,41.5-43 \mathrm{~cm} ; 759.7$ to 605.5 mbsf) is the stratigraphically youngest DWAF assemblage in Hole 909C. The lower boundary of the assemblage is close to a major faunal density minimum (text-fig. 3). This assemblage was identified by Osterman and Spiegler up to Core 909C-55R-CC. We extend the upper boundary of this assemblage upwards to Core 909C-55R-1, where the LO of Reticulophragmium rotundidorsatum (Hantken 1875) is observed. The faunal abundance and diversity declines upsection through the middle to upper Miocene, and above Core -55R the samples are barren of foraminifera. This assemblage contains the LOs of Reticulophragmium amplectens (Grzybowski 1898), Karreriella seigliei (Gradstein and Kaminski 1989), Repmanina charoides (Jones and Parker 1860), Pseudonodosinella elongata (Grzybowski 1898), Psamminopelta gradsteini Kaminski and Geroch 1997, and Spirosigmoilinella compressa Matsunaga 1955.

\section{Palaeobathymetry and Paleoecology}

The study of agglutinated foraminiferal morphogroups has much potential for palaeoenvironmental interpretation, as the life position and the relative abundance of trophic groups are affected by various environmental parameters, such as organic productivity and bottom-water oxygenation. As pointed out by Jones and Charnock (1985); Nagy (1992); Nagy et al. (1995, 1997); and van den Akker et al. (2000), agglutinated foraminifera taxa can be grouped into "morphotypes" based on gross morphology that are believed to reflect differences in the life position and feeding habit. Morphotypes in turn can be combined into "morphogroups", according to their preferred habitat with respect to the sediment/water interface (e.g., infaunal vs. epifaunal morphogroups). The morphogroup classification used here (text-figure 4) is based on the work of Nagy et al. (1997) and van den Akker et al. (2000). There is one difference between the study of van den Akker et al. (2000) and our morphogroups. In this study, the species Aschemocella sp., and Aschemocella subnodosiformis are interpreted as belonging to morphogoup M1 of the tubular forms, whilst the single chambers of Aschemocella grandis (only a few specimens have been found) are included into morphotype M2a of the globular forms.

The agglutinated foraminiferal species in Hole 909C comprise seven morphotypes belonging to four main groups (text-figure 4). The relative proportions of the morphotypes in Hole 909C are presented in text-figure 5 . The tubular taxa of morphogroup 1 (M1) are common throughout the studied sedimentary sequence, representing about $30-40 \%$ of the total number assemblage, with a lower (10-20\%) proportion in the uppermost part of the studied interval (above 762 mbsf). Nagy et al. (1997) interpreted variations in this morphogroup as a paleobathy- 


\begin{tabular}{|c|c|c|c|c|c|c|}
\hline MORPHOTYPE & $\begin{array}{c}\text { TEST } \\
\text { SHAPE }\end{array}$ & MAIN GENERA & $\begin{array}{l}\text { MORPHO } \\
\text {-GROUP }\end{array}$ & $\begin{array}{c}\text { LIFE } \\
\text { POSITION }\end{array}$ & $\begin{array}{c}\text { FEEDING } \\
\text { HABIT }\end{array}$ & ENVIRONMENT \\
\hline & Tubular & $\begin{array}{c}\text { Aschemocella } \\
\text { Bathysiphon } \\
\text { Hyperammina } \\
\text { Nothia } \\
\text { Rhabdammina } \\
\text { Rhizammina } \\
\text { Psammosiphonella } \\
\end{array}$ & M1 & Erect epifauna & $\begin{array}{l}\text { Suspension } \\
\text { feeding }\end{array}$ & $\begin{array}{l}\text { Tranquil bathyal and } \\
\text { abyssal with low } \\
\text { organic matter flux }\end{array}$ \\
\hline & Globular & $\begin{array}{l}\text { Lagenammina } \\
\text { Psammosphaera } \\
\text { Saccammina }\end{array}$ & M2a & $\begin{array}{l}\text { Shallow } \\
\text { infauna }\end{array}$ & $\begin{array}{c}\text { Suspension } \\
\text { feeding } \\
- \\
\text { Passive deposit } \\
\text { feeding }\end{array}$ & $\begin{array}{l}\text { Common in bathyal } \\
\text { and abyssal }\end{array}$ \\
\hline & $\begin{array}{l}\text { Rounded } \\
\text { trochospiral } \\
\quad \text { and } \\
\text { streptospiral }\end{array}$ & $\begin{array}{l}\text { Budashevaella } \\
\text { Cribrostomoides } \\
\text { Recurvoidella } \\
\text { Recurvoides }\end{array}$ & \multirow[t]{2}{*}{ M2b } & \multirow[t]{2}{*}{$\begin{array}{l}\text { Surficial } \\
\text { epifauna }\end{array}$} & \multirow[t]{2}{*}{$\begin{array}{l}\text { Active deposit } \\
\text { feeding }\end{array}$} & \multirow[t]{2}{*}{ Shelf to deep marine } \\
\hline & $\begin{array}{l}\text { Planoconvex } \\
\text { trochospiral }\end{array}$ & $\begin{array}{l}\text { Conotrochammina } \\
\text { Trochammina }\end{array}$ & & & & \\
\hline \multirow[t]{2}{*}{$\longrightarrow$} & $\begin{array}{l}\text { Elongate } \\
\text { keeled }\end{array}$ & & M2c & $\begin{array}{l}\text { Surficial } \\
\text { epifauna }\end{array}$ & $\begin{array}{l}\text { Active deposit } \\
\text { feeding }\end{array}$ & $\begin{array}{l}\text { Shelf to marginal } \\
\text { marine }\end{array}$ \\
\hline & $\begin{array}{l}\text { Flattened } \\
\text { trochospiral }\end{array}$ & & \multirow[b]{2}{*}{ M3a } & \multirow[b]{2}{*}{$\begin{array}{l}\text { Surficial } \\
\text { epifauna }\end{array}$} & \multirow[b]{2}{*}{$\begin{array}{l}\text { Active and } \\
\text { passive deposit } \\
\text { feeding }\end{array}$} & \multirow[b]{2}{*}{ Lagoonal to abyssal } \\
\hline & $\begin{array}{c}\text { Flattened } \\
\text { planispiral and } \\
\text { streptospiral }\end{array}$ & $\begin{array}{c}\text { Ammodiscus } \\
\text { Ammosphaeroidina } \\
\text { Eratidus } \\
\text { Glomospira } \\
\text { Psamminopelta } \\
\text { Spirosigmoilinella } \\
\end{array}$ & & & & \\
\hline$=$ & $\begin{array}{l}\text { Flattened } \\
\text { irregular }\end{array}$ & Ammolagena & M3b & $\begin{array}{l}\text { Surficial } \\
\text { epifauna }\end{array}$ & $\begin{array}{l}\text { Passive deposit } \\
\text { feeding }\end{array}$ & $\begin{array}{c}\text { Upper bathyal to } \\
\text { abyssal }\end{array}$ \\
\hline & $\begin{array}{l}\text { Rounded } \\
\text { planispiral }\end{array}$ & $\begin{array}{c}\text { Cyclammina } \\
\text { Haplophragmoides } \\
\text { Reticulophragmium } \\
\text { Veleroninoides }\end{array}$ & M4a & $\begin{array}{c}\text { Surficial } \\
\text { epifauna } \\
- \\
\text { Shallow } \\
\text { infauna }\end{array}$ & $\begin{array}{l}\text { Active deposit } \\
\text { feeding }\end{array}$ & $\begin{array}{l}\text { Inner shelf to upper } \\
\text { bathyal }\end{array}$ \\
\hline$\geqslant$ & $\begin{array}{c}\text { Elongate } \\
\text { subcylindrical }\end{array}$ & $\begin{array}{l}\text { Karrerulina } \\
\text { Karreriella }\end{array}$ & \multirow[b]{2}{*}{ M4b } & \multirow[b]{2}{*}{ Deep infauna } & \multirow[b]{2}{*}{$\begin{array}{l}\text { Active deposit } \\
\text { feeding }\end{array}$} & \multirow[b]{2}{*}{$\begin{array}{l}\text { Inner shelf to upper } \\
\text { bathyal with increased } \\
\text { organic matter flux }\end{array}$} \\
\hline & $\begin{array}{l}\text { Elongate } \\
\text { tapered }\end{array}$ & $\begin{array}{l}\text { Ammobaculites } \\
\text { Hormosinella } \\
\text { Reophanus } \\
\text { Reophax } \\
\text { Subreophax } \\
\text { Pseudonosinella } \\
\end{array}$ & & & & \\
\hline
\end{tabular}

TEXT-FIGURE 4

Morphogroup classification of agglutinated foraminifera found in Hole 909C (modified after van der Akker et al. 2000).

metrical indicator, as the tubular forms are most abundant in continental slope and rise settings where particulate organic matter is advected by gentle bottom currents. If this interpretation holds, the high proportions of M1 recovered at the base of the drilled section imply that Site 909 had already subsided to lower bathyal depths by the early Miocene. Based on plate tectonic reconstructions, Kristofferson (1990) speculated that shallow-water connections through the Fram Strait had already opened by the mid Oligocene, subsiding to about $2 \mathrm{~km}$ depth by the middle Miocene. Our faunal data are in agreement with this estimate. The first appearance of cosmopolitan benthic foram- inifera in the upper Eocene to lower Oligocene of the Beaufort Sea wells led McNeil (1990) to postulate a somewhat earlier connection to the Atlantic Ocean.

Morphotype M2b (dominated by Recurvoides and related forms) shows little overall trend, but has three broad maxima of around $20 \%$ of the total fauna within intervals with lower proportions of M1. Recurvoides is a cosmopolitan genus, typical of deep-water settings. Recurvoides is regarded by Gradstein et al. (1994) as a typical indicator for middle and lower bathyal facies in the North Sea. 
The most striking feature of the morphogroup analysis is the trend displayed by morphotype M4a (shallow infauna and surficial epifauna). This morphotype shows an inverse trend to morphogroup M1, steadily increasing from an average of $20-30 \%$ of the assemblage below $762 \mathrm{mbsf}$, to $60-80 \%$ in samples above $660 \mathrm{mbsf}$. In the Paleogene sections of Spitsbergen and the Barents Sea area, high abundances of this morphotype are regarded by Nagy et al. (2000) as typical of shallower depositional environments at middle to upper neritic water depths. While we do not suggest here that the Fram Strait sill experienced any significant tectonic uplift, it is important to note that shallower depositional settings typically experience higher rates of organic carbon flux. Therefore, it is more likely that morphotype M4a is responding to changes in organic productivity rather than paleobathymetry.

The record of organic carbon values in Hole 909C reflect changes in the supply of transported terrestrial organic matter and the flux of marine organic matter from primary production. The high TOC values at the base of Hole 909C reflect high amounts of terrestrially-derived organic matter from the surrounding land masses (Knies and Mann 2002). In this part of the section the proportion of morphotype M4a is small. Above $920 \mathrm{mbsf}$ the proportion of morphotype M4a increases concurrently with an increase in the percent TOC (values taken from Stein and Stax 1996). The TOC values increase from an average of $0.8 \%$ in Core $-70 \mathrm{R}$ (ca. $750 \mathrm{mbsf}$ ) to values as high as 1.5 $\%$ in Core $-55 \mathrm{R}$ (ca. $605 \mathrm{mbsf}$ ). The low $\mathrm{C} / \mathrm{N}$ values of the organic carbon over this interval (between 7 and 9) indicate a strong marine component (Stein and Stax 1996). Stein and Stax remarked that the unusually high flux of marine organic carbon at Site 909 requires paleoceanographic conditions that are "different from normal open-ocean ones."

It is noteworthy that the observed shift in the proportions of the morphotypes M1, M2a, and M4a at 762mbsf coincides with the reappearance of Reticulophragmium rotundidorsatum (and the establishment of our Reticulophragmium rotundidorsatum Assemblage), suggesting that the distribution of the Reticulophragmium rotundidorsatum is environmentally controlled. This fact becomes evident considering that in the lower part of the section, $R$. rotundidorsatum is present in only a few samples and in lower proportions (text-fig. 3) than in the upper part of the section, above $762 \mathrm{mbsf}$. This fact suggests that Reticulophragmium rotundidorsatum is sensitive to organic flux levels or other parameters of the deep ocean environment.

\section{DISCUSSION and CONCLUSIONS}

The stratigraphic importance of the Miocene agglutinated foraminiferal fauna recovered from Hole 909C cannot be over-emphasized. Not only is Site 909 situated in a key oceanic gateway, but DWAF species that are elsewhere recorded from Paleogene sediments range into stratigraphically younger strata at this locality. Osterman and Spiegler (1996) recorded several species (e.g., Hyperammina rugosa, Nothia excelsa, Haplophragmoides porrectus) for the first time from Miocene deposits. In this study we document the youngest known occurrences of some additional species, such as Reophanus berggreni and Psamminopelta gradsteini (text-fig. 2; Appendix 1). In a study of the Paleogene assemblages from ODP Hole 643A, Kaminski et al. (1990) first pointed out the apparent diachrony of high-latitude agglutinated foraminifera with increasing latitude. Schröder-Adams and McNiel (1994) also pointed out that a number of cosmopolitan species such as $R$. amplectens, $B$. multicamerata, $R$. rotundidorsatum and $S$. compressa became extinct later (in the Miocene) in the Beaufort Sea-MacKenzie Delta area than in the North Atlantic region.

In the North Atlantic petroleum basins, Paleocene to Eocene assemblages are predominantly agglutinated, Oligocene to Miocene assemblages usually contain varying proportions of calcareous and agglutinated taxa, while the Miocene to Recent assemblages are predominantly calcareous (Gradstein et al. 1994). This general faunal change has been widely interpreted as representing the transition from the silled, stratified basins of the Paleogene to more well oxygenated basins that allowed the preservation of biogenic carbonate (Gradstein and Berggren 1981). According to this model, deep, cold, stratified basins with high sedimentation rates favour preservation of the DWAF assemblages. This is also the case with the anomalously young Oligocene-Miocene DWAF assemblages documented by Schröder-Adams and McNeil (1994) from the Beaufort Sea.

Osterman and Spiegler (1996) suggested a strong environmental control of the DWAF assemblages from Hole 909C to explain their occurrence in younger strata. They noted that the optimum habitat of the DWAF possibly shifted northward, as rifting in the North Atlantic progressed, creating high sedimentation-rate basins. The Miocene sediments at Site 909 are unusual in that they are organic rich, largely non-calcareous, and reflect higher sedimentation rates than at other sites further to the south. Furthermore, it may be the case that because of the tectonic evolution of the Fram Strait and other sills further to the south, this region was isolated from North Atlantic bottom waters during the Miocene. Because of this isolation and suitable environmental conditions (without carbonate deposition), the Paleogene DWAF faunas found a refuge in the Fram Strait region up to the late Miocene (Osterman and Spiegler 1996). However, there is a need for further paleobiogeographical studies (including comparisons with microfossil assemblages from the Beaufort Sea and the Arctic region) to fully clarify the problem of diachrony among agglutinated assemblages.

At present, the general oceanic circulation pattern between the Arctic Nansen Basin and the Greenland Sea is estuarine, with surface waters flowing out of the Arctic and warmer, more saline water of Atlantic origin flowing into the Nansen Basin at depth. The presence of DWAF with Norwegian Sea affinities at Site 909 strongly suggests that this estuarine circulation pattern was in place by the Early Miocene. However, we need to compare the record from Site 909 with the new data obtained from the Arctic Drilling Expedition (summer 2004) to fully resolve this issue.

Today, the area of the comparatively warm, seasonally ice-free West Spitsbergen Current to the west and north of Spitsbergen is the most highly productive sector of the European Arctic region. Productivity declines rapidly to the west and to the north of the current as the amount of ice cover increases, with highly oligotrophic conditions present in the permanently ice-covered Central Arctic Ocean (Wollenburg and Mackensen 1998). The morphogroup analysis and sediment parameters from Hole 909C suggest that paleoenvironmental conditions in the Fram Strait area were not stable during the Miocene. A shift in the proportions of the agglutinated foraminiferal morphotypes is observed alongside changes in the organic carbon flux at Site 909 , in particular, the proportion of the planispiral morphotype M4a appears to be positively correlated with the TOC content.

Stein and Stax (1996) speculated that variations in the West Spitsbergen Current and in sea-ice cover through time may have 


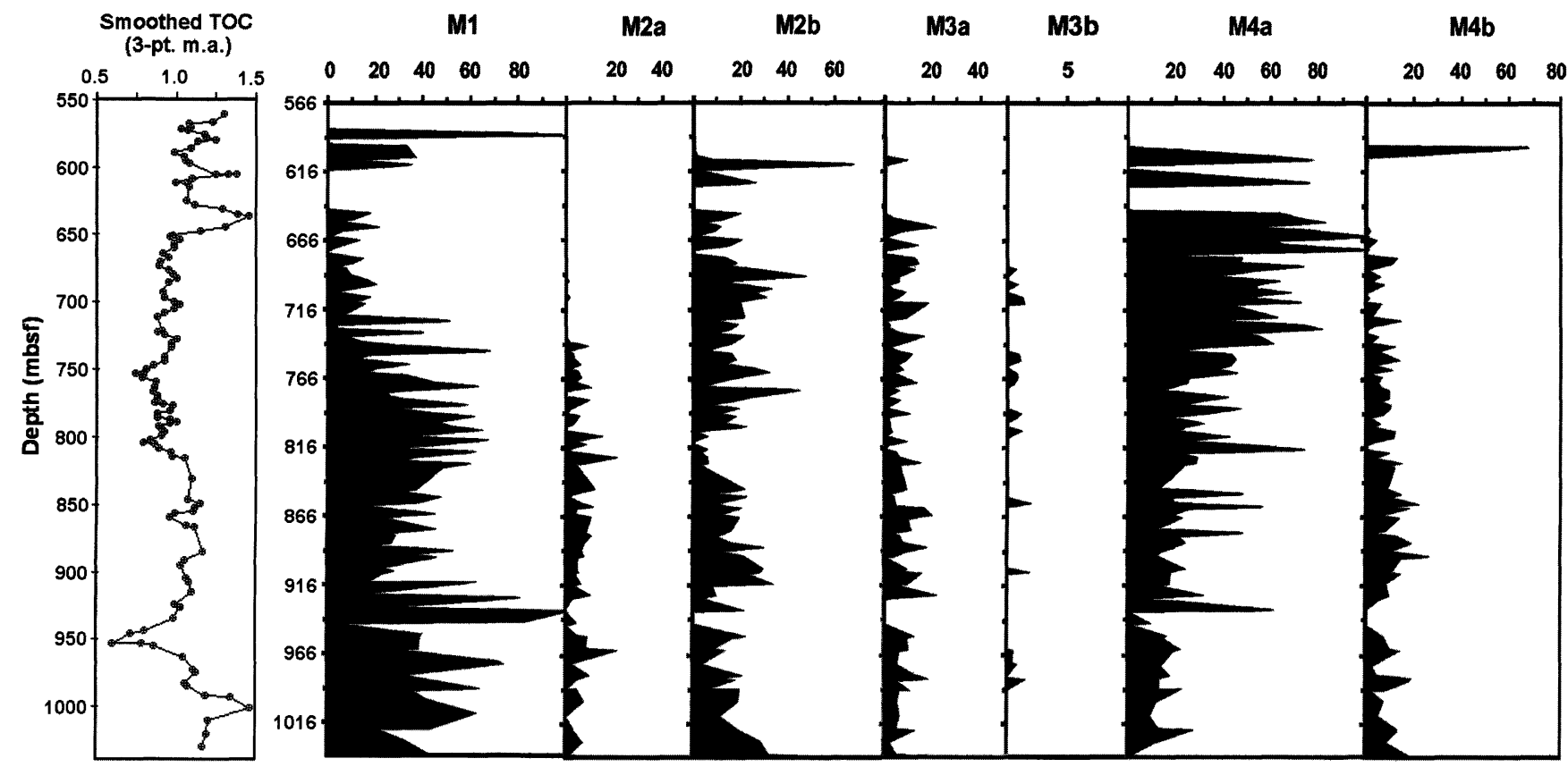

TEXT-FIGURE 5

Relative distribution (in percentage) of agglutinated foraminiferal morphotypes in Hole 909C (566.97-1040.71 mbsf). Also shown is the 3-point moving average of percent Total Organic Carbon values in the studied section (data from Stein and Stax 1996).

been the dominant mechanism controlling the organic carbon deposition at Site 909, which we think also influenced the proportions of the benthic foraminiferal morphotypes. Site 909 is located on the bathymetric sill between the Norwegian-Greenland Sea and the Arctic Ocean, currently at ca. $2600 \mathrm{~m}$ depth. Allowing for normal thermal subsidence, the site would have been shallower during the Miocene, and affected by surface and intermediate waters of Atlantic origin. Any intensification of the surface and intermediate water exchange over the Fram Strait sill would have created locally increased upwelling conditions, leading to enhanced primary productivity. The location of the ice margin is also important because of the occurrence of ice-margin phytoplankton blooms (Smith and Nelson 1986). The record of dinoflagellate cysts from Hole 909C (Poulsen et al. 1996; fig. 3) shows increasing numbers of heterotrophic forms such as Selenopemphix, Protoperidinium, and Lejeunecysta (forms that feed upon diatoms) over the same interval. However, the micropalaeontological residues are devoid of any planktonic microfossils, so it is not clear which planktonic organisms contributed to the productivity signal at Site 909.

Winkler et al. (2002) noted a change in the clay minerals and an increase in bulk accumulation rate over this same interval in Hole 909C, suggesting a provenance change in the terrigenous component. At the same time, an increase in coal fragments, igneous and metamorphic rock fragments and mica content occur (Wolf-Welling et al. 1996). These sedimentological changes are regarded as reflecting an increase in the water mass exchange through the Fram Strait as well as seasonal ice rafting (Winkler et al. 2002). Bohrmann et al. (1990) noted an intensification of bottom currents in the Vøring Plateau region (Sites 642 and 643) between 13 and $11 \mathrm{Ma}$, and linked this to the establishment of significant outflow over the Iceland-Scotland Ridge.
The only other locality in the northern North Atlantic where DWAF assemblages were documented from upper Miocene sediments is at ODP Site 646 off the southern tip of Greenland (Kaminski et al. 1989). In Hole 646A, the agglutinated assemblage occurs in the interval with the highest bulk sediment accumulation rate and TOC contents (see Bohrmann et al. 1990, fig. 6). This interval in Hole 646 was linked to the appearance of Denmark Strait Overflow water at this locality at approximately 7.5 Ma. Interestingly, the DWAF assemblages disappeared from the record of Hole 646A when biosiliceous sedimentation commenced around 4 Ma (Kaminski et al. 1989; Bohrmann et al. 1990).

We conclude that the "favorable" environment for the occurrence of Neogene DWAF assemblages in the northern North Atlantic involves the rapid accumulation of carbonate-poor terrigenous sediments, moderate (not high) rates of organic carbon flux, and the absence of a significant biosiliceous component. The presence of gentle bottom currents probably also plays a positive role, especially with regards to the suspension feeding tubular morphogroup. At other Norwegian Sea sites where Miocene sediments are biosiliceous, the DWAF assemblages are either absent or consist of only a few specialized taxa (Verdenius and Van Hinte 1983, Kaminski et al. 1990). As pointed out by previous authors (e.g., Gradstein and Berggren 1981; Ostermann and Spiegler 1996) such favorable sedimentary conditions can be found in the tectonically active high-latitude deep basins. The diversity of species and morphogroups in Miocene DWAF assemblages appears to be a function of the trophic continuum, and in particular the carbon flux (Preece et al. 1999). Diversity is negatively correlated with carbon flux, with larger numbers of agglutinated species occurring towards the oligotrophic end of the trophic spectrum (Altenbach et al. 1999). 


\section{ACKNOWLEDGMENTS}

We thank the Ocean Drilling Program Core Repository for providing samples from Hole 909C, and the NERC ODP committee for funding the current project (Grant NER/T/S/ 2003/00037). Lóránd Silye thanks the EEC Socrates Programme and the Grzybowski Foundation (the Stanislaw Geroch Memorial Scholarship Fund) for sponsoring his studies at UCL. We thank Thomas M. Cronin (USGS), Jens Matthiessen (AWI), and John Farrell (URI) for kindly pre-reviewing the paper, and Rachel Preece (Chevron) and Wolfgang Kuhnt (CAU) for reviewing the final draft. This is contribution number 75 of the Deep-Water Agglutinated Foraminiferal Project.

\section{TAXONOMIC NOTES}

The taxa below are listed alphabetically by genus. Species identifications are based largely on the taxonomic work of Gradstein and Kaminski (1989, 1997), Charnock and Jones (1990), Kaminski and Geroch (1993), Gradstein et al. (1994), Kaminski et al. (1988, 1989, 1990), Schröder-Adams and McNeil (1994), Osterman and Spiegler (1996), Evans and Kaminski (1998), Green et al. (2004), and Kaminski and Gradstein (2005). For the sake of brevity, the synonymy lists only primary references, revisions of primary types, and/or modified or tentative identifications.

? Ammobaculites jarvisi Cushman and Renz 1946

Plate 4, figure 2

? Ammobaculites jarvisi CUSHMAN and RENZ 1946, p. 19, pl. 2, figs. 8,9 .

Ammodiscus incertus d'Orbigny 1839

Ammodiscus incertus D'ORBIGNY 1839, p. 49.

Ammodiscus peruvianus Berry 1928

Plate 1, figure 20

Ammodiscus peruvianus BERRY 1928, p. 342, pl. 27.

Ammodiscus tenuissimus Grzybowski 1898

Plate 1, figure 21

Ammodiscus tenuissimus GRZYBOWSKI 1898, p. 282, pl. 10, fig. 35.
Ammodiscus cf. A. latus Grzybowski

cf. Ammodiscus latus GRZYBOWSKI 1898, p. 282, pl. 10, figs. 27-28.

Fragments are closest to Ammodiscus latus Grzybowski

\section{Ammodiscus sp. 1}

Plate 2, figure 2

Deformed or poorly preserved specimens, which are closest to Ammodiscus tenuissimus Grzybowski.

\section{Ammodiscus sp. 2}

Plate 2, figure 1

Broken specimen resembling Ammodiscus cretaceus (Reuss).

\section{Ammodiscus spp.}

Deformed or poorly preserved specimens with no affinities to known species of Ammodiscus.

\section{Ammolagena clavata (Jones and Parker 1860)}

Plate 2, figures $3 \mathrm{a}, \mathrm{b}, 4$

Trochammina irregularis (d'Orbigny) var. clavata JONES and PARKER 1860, p. 304.

Ammolagena clavata (Jones and Parker 1860).- KAMINSKI and GRADSTEIN 2005, p. 165, pl.21, figs. 1-6 (figure 1 is the lectotype).

\section{Ammolagena sp.}

Plate 2, figure 5

Broken specimen of Ammolagena clavata without a visible proloculus.

\section{Ammosphaeroidina sp.}

Plate 4, figure 5a,b,c

?Cystaminella pseudopauciloculata MJATLIUK 1966, p. 264, pl. 1, figs. 5-7; pl. 2, fig. 6; pl. 3, fig. 3 .

Specimens are closest to Ammosphaeroidina pseudopauciloculata (Mjatliuk).

\section{PLATE 1}

1-2 Rhabdammina abyssorum [64R-3, 40-41.5cm] $860 \mu \mathrm{m}, 924 \mu \mathrm{m}$

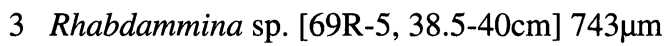

4 Bathysiphon sp. 1 [68R-3, 40-41.5cm] 600 $\mu \mathrm{m}$

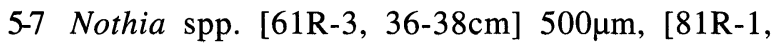
$40.5-42 \mathrm{~cm}] 1321 \mu \mathrm{m},[72 \mathrm{R}-3,37.5-39 \mathrm{~cm}] 822 \mu \mathrm{m}$

8 Nothia excelsa [69R-5, 38.5-40cm] $559 \mu \mathrm{m}$

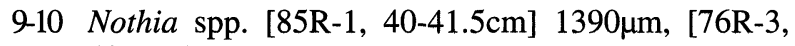
$40-41.5 \mathrm{~cm}] 770 \mu \mathrm{m}$

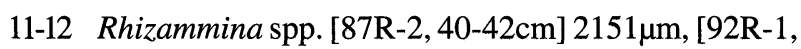
$38.5-40.5 \mathrm{~cm}] 244 \mu \mathrm{m}$
13 Lagenammina sp. [71R-3, 38-39.5cm] $518 \mu \mathrm{m}$

14 Lagenammina sp. [80R-1, 40-41.5cm] $517 \mu \mathrm{m}$

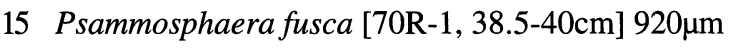

16 Hyperammina elongata [69R-1, 38.5-40cm] $866 \mu \mathrm{m}$,

17 Hyperammina rugosa [91R-2, 38-40cm] $376 \mu \mathrm{m}$

18-19 Hyperammina elongata [73R-3, 38.5-40cm] 906 $\mu \mathrm{m}$, [91R-2, 38-40cm] $1162 \mu \mathrm{m}$

20 Ammodiscus peruvianus [64R-3, 40-41.5cm] 223 $\mu \mathrm{m}$

21 Ammodiscus tenuissimus [64R-3, 40-41.5cm] $255 \mu \mathrm{m}$. (If not labeled, scale bar $=400 \mu \mathrm{m}$ ). 


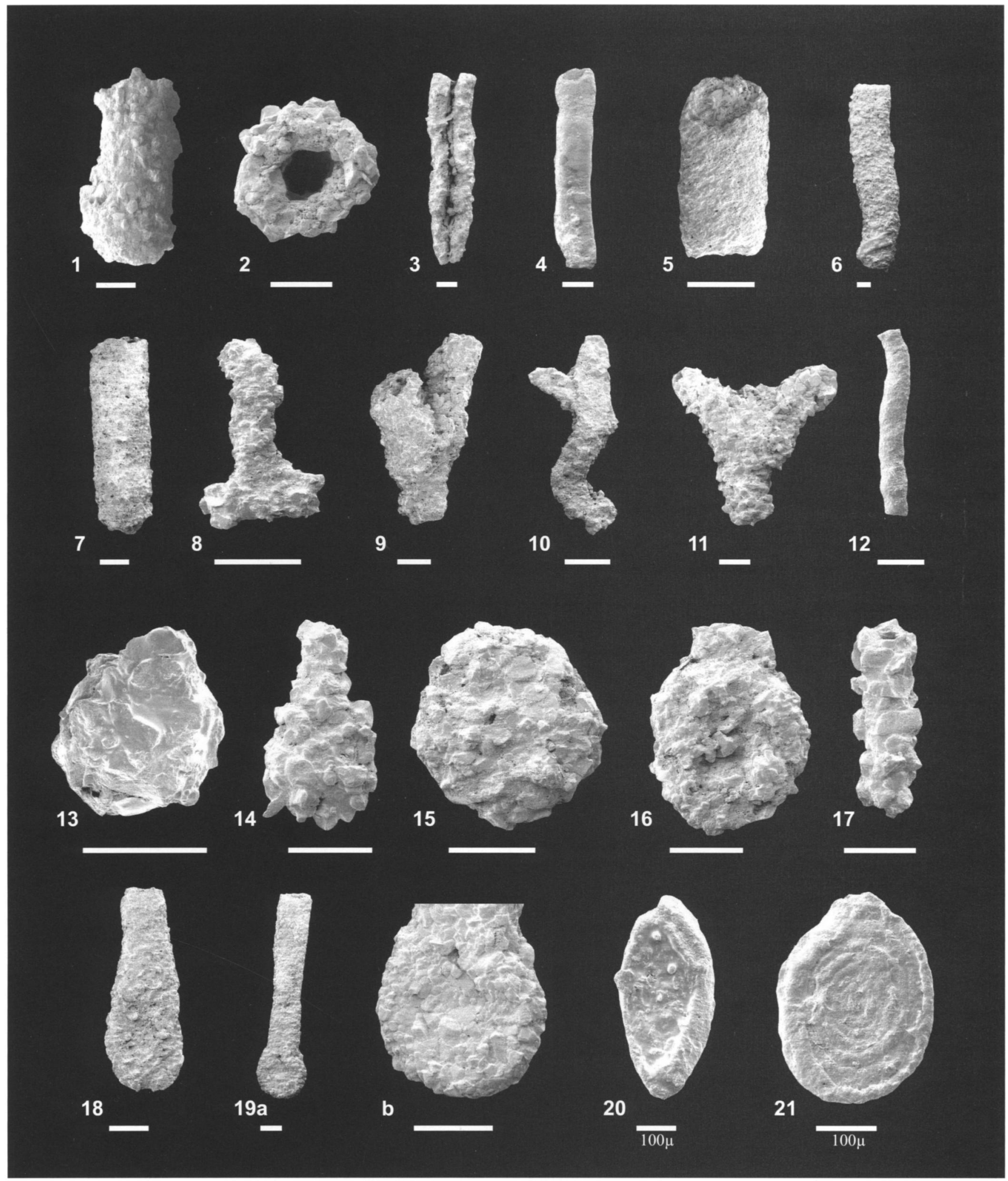


Aschemocella grandis (Grzybowski 1898)

Reophax grandis GRZYBOWSKI 1898, p. 277, pl. 10, figs 13-15.

Aschemocella subnodosiformis (Grzybowski 1898).- KAMINSKI and GEROCH 1993, p. 249, pl. 2, figs. 8-10.

Aschemocella sp.

Deformed and broken chambers resembling Aschemocella subnodosiformis (Grzybowski 1898).

Aschemocella subnodosiformis (Grzybowski 1898)

Hyperammina subnodosiformis GRZYBOWSKI 1898, p. 274, pl. 10, figs 5-6.

Aschemocella subnodosiformis (Grzybowski 1898).- KAMINSKI and GEROCH 1993, p. 249, pl. 1, figs. 10a-13.

Bathysiphon sp. 1

Plate 1, figure 4

Fragments of elongate cylindrical tubes, thin walled and finely agglutinated.

\section{Bathysiphon sp. 2}

Similar to Bathysiphon sp. 1, but about three times wider.

Bathysiphon sp. 3

Similar to Bathysiphon sp. 1, but about twice the width.

Budashevaella multicamerata (Voloshinova and Budasheva 1961) Plate 4, figure 6a,b

Circus multicameratus VOLOSHINOVA and BUDASHEVA 1961, p. 201, pl. 7 figs. 6a-c, pl. 7, figs. 4-5.

Budashevaella sp. 1

Specimen are closest to Budashevaella multicamerata (Voloshinova and Budasheva 1961).

\section{Budashevaella sp. 2}

Includes smaller specimens with about 7-8 chambers in the last whorl, oval in outline, which resemble the Paleocene species $B$. trinitatensis.

\section{Conotrochammina sp.}

Plate 5, figure 3a,b,c

Fine grained specimens, belonging to genus Conotrochammina.

\section{Cribrostomoides spp.}

Includes mostly deformed specimens that are probably closest to C. subglobosus (Cushman 1910).

Cyclammina placenta (Reuss 1851)

Nonionina placenta REUSS 1851, p. 72, fig. 33a,b.

Cyclammina placenta (Reuss).- GRADSTEIN et al. 1994, pl. 6, figs 7-11.

Includes specimens with slightly sigmoidal final sutures that are transitional to $C$. cancellata.

Cyclammina cancellata Brady 1879

Cyclammina cancellata BRADY 1879, p. 62.- BRADY 1884, pl. 37, figs 8-15.

The largest Cyclammina in our material, with sigmoidal sutures and up to 16 chambers in the final whorl.

Cyclammina sp.

Plate 7 , figure $3 a, b, c$

Smaller than $C$. cancellata, with a subacute periphery and only a few supplementary apertures.

Eratidus sp.

Plate 4, figures 3, 4

Specimens are smaller and have a narrower uniserial portion compared with the modern species E. foliaceus (Brady).

\section{Glomospira spp.}

Specimens most closely resemble Glomospira gordialis (Jones and Parker).

Haplophragmoides $\boldsymbol{c f}$. H. bradyi (Robertson 1891)

cf. Trochammina bradyi ROBERTSON 1891, p. 388.

\section{PLATE 2}

1 Ammodiscus sp. 2 [78R-1, 40-42cm] 1536 $\mu \mathrm{m}$

2 Ammodiscus sp. 1 [87R-1, 40-42cm] 362 $\mu \mathrm{m}$

3 Ammolagena sp. attatched to Reticulophragmium sp. [70R-3, 37.5-39.5cm] 596 $\mu \mathrm{m}$

4 Ammolagena clavata attatched to Cyclammina sp. [75R-5, 40-41.5cm] $1800 \mu \mathrm{m}$

5 Ammolagena sp. attatched to Nothia sp. [81R-1, $40.5-42 \mathrm{~cm}] 1321 \mu \mathrm{m}$

6 Rhizammina sp. (Tolypammina vagans of Spiegler) [72R-1, 37.5-39cm] $612 \mu \mathrm{m}$

7-8 Repmanina charoides [68R-4, 38.5-40cm] 341 $\mu \mathrm{m}$, [62R-5, 38-40cm] $327 \mu \mathrm{m}$
9-11 Psamminopelta gradsteini [94R-1, 40-41.5cm] $382 \mu \mathrm{m},[95 \mathrm{R}-1,42-43.5 \mathrm{~cm}] 174 \mu \mathrm{m},[61 \mathrm{R}-5,37.5-$ $39 \mathrm{~cm}] 455 \mu \mathrm{m}$

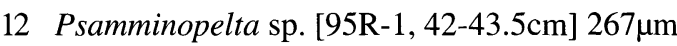

13 Spirosigmoilinella compressa [60R-1, 38.5-40cm] $504 \mu \mathrm{m}$

14-15 Hormosinella carpenteri [67R-3, 40-41.5cm] $1467 \mu \mathrm{m},[94 \mathrm{R}-3,35-36.5 \mathrm{~cm}] 1826 \mu \mathrm{m}$

16-18 Reophanus berggreni [94R-3, 35-36.5cm] 2259 $\mu \mathrm{m}$, [93R-1, 40-41.5cm] 2000 $\mu \mathrm{m}$, [94R-1, 40-41.5cm] $2032 \mu \mathrm{m}$. (If not labeled scale bar $=400 \mu \mathrm{m}$ ). 


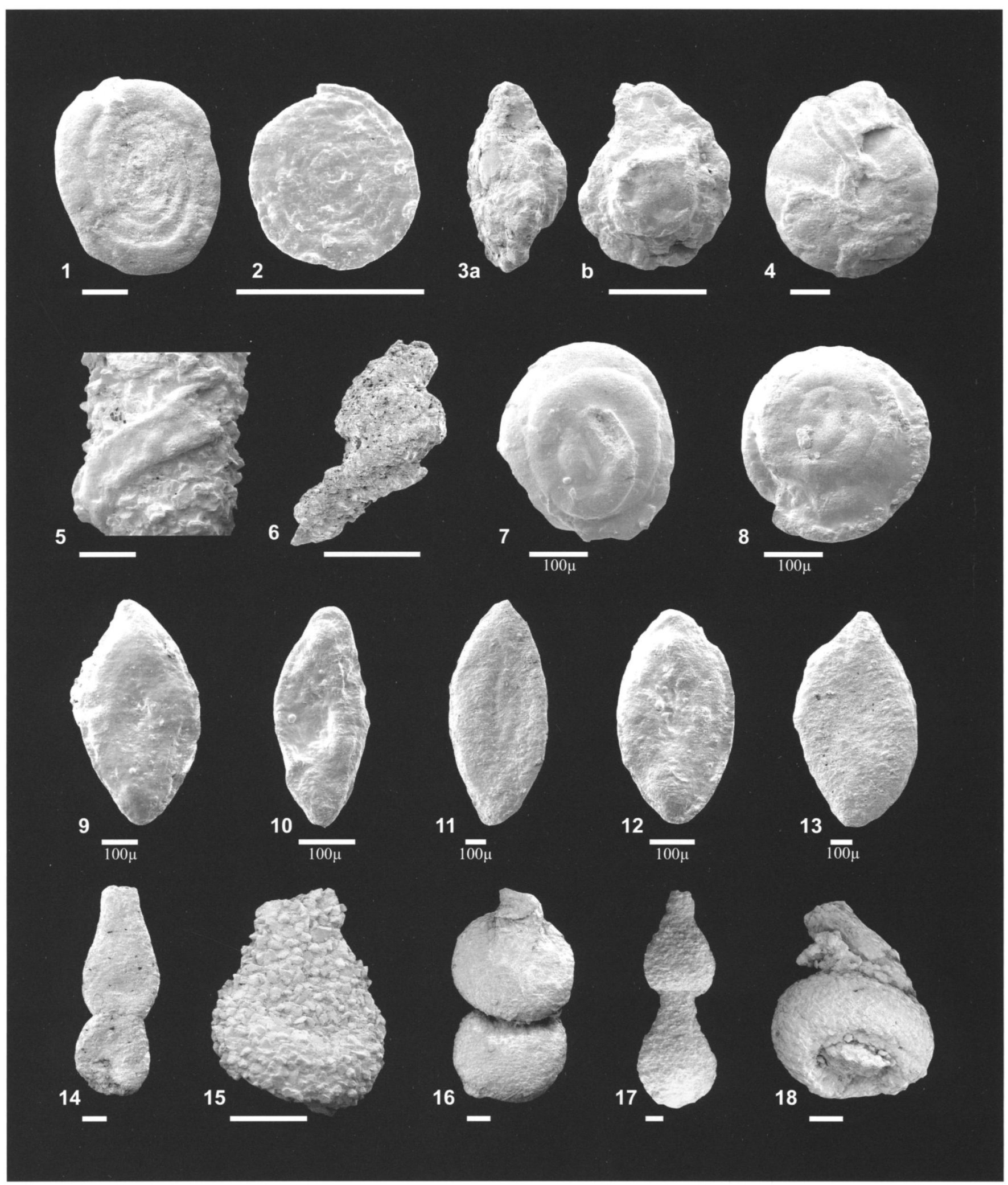


Haplophragmoides cf. H. bradyi (Robertson 1891).- GREEN et al. 2004, p. 124, pl. 1, figs. 5-7.

Slightly larger than the typical modern form. It most closely resembles the specimens from the Gulf of Mexico illustrated by Green et al. (2004).

Haplophragmoides ex gr. H. carinatus Cushman and Renz 1941 Plate 3, figures 12a,b,c, 14a,b, 15a,b

Haplophragmoides carinatus CUSHMAN and RENZ 1941, p. 2, pl. 1, fig. 1.- SCHRÖDER-ADAMS and MCNEIL 1994, p. 38, pl. 4, figs. 9-13.

The morphology of the species is quite variable, and some transitional forms may be present. We use the term in a broad sense, following Schröder-Adams and McNeil (1994).

Haplophragmoides walteri/carinatus transitional form Plate 3, figure 11

Transitional forms between $H$. carinatus and $H$. walteri.

Haplophragmoides eggeri Cushman 1926

Haplophragmoides eggeri CUSHMAN 1926, p. 583, pl. 15, figs. 1a,b.

Haplophragmoides aff. H. horridus (Grzybowski 1901)

aff. Haplophragmium horridum GRZYBOWSKI 1901, p. 270, pl. 7, fig. 12.

Our specimens have the distinctive triangular last chamber of $H$. horridus, but differ in having only 5 chambers in the last whorl.

\section{Haplophragmoides sp. 1}

Plate 3, figure 16a,b

Flattened specimens that have fewer chambers than $H$. carinatus.

\section{Haplophragmoides sp. 2}

Plate 3, figure 13

Specimens that most closely resemble $H$. walteri, but have a partial carina. Possibly transitional to $H$. carinatus.

\section{Haplophragmoides sp. 3}

Deformed specimens that are closest to $R$. pusillum.

\section{Haplophragmoides sp. 4}

These are mostly flattened specimens with a thin wall and 4-5 chambers, which most closely resemble $H$. bradyi (Robertson 1891).

\section{Haplophragmoides sp. 5}

Flat specimens with more than 5 chambers, which most closely resemble Haplophragmoides stomatus (Grzybowski).

\section{Haplophragmoides sp. 6}

A large, flat, thin-walled form with a round open umbilicus and 6 to $61 / 2$ chambers.

\section{Haplophragmoides spp.}

Plate 4, figure 1a,b

Includes the broken, deformed specimens whose affinity to genus Haplophragmoides is only tentative.

Haplophragmoides walteri (Grzybowski 1898)

Plate 3, figure 10a,b

Trochammina walteri GRZYBOWSKI 1898, p. 290, pl. 11, fig. 31.

Haplophragmoides walteri (Grzybowski).- KAMINSKI and GEROCH 1993 , p. 263, pl. 10, fig. 3a-7c.

Hormosinella carpenteri (Brady 1881)

Plate 2, figures 14, 15

Trochammina (Hormosina) carpenteri BRADY 1881, p. 51

Hormosina carpenteri BRADY 1884, p. 327, pl. 39, figs. 14-18.

Specimens have large, irregular chambers.

Hormosinella sp.

The specimens are closest to Hormosinella carpenteri.

Hyperammina elongata Brady 1878

Plate 1, figures 16, 18, 19a,b

Hyperammina elongata Brady 1878.- OSTERMAN and SPIEGLER 1996, pl. 1, fig. 6 .

Hyperammina rugosa Verdenius and Van Hinte 1983

Plate 1, figure 17

Hyperammina rugosa VERDENIUS and VAN HINTE 1983, p. 187, fig. 16; pl. 1, figs. 12-14.

\section{PLATE 3}

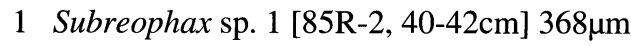

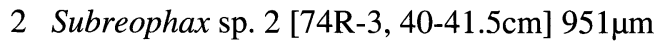

3-4 Pseudonodosinella nodulosa [61R-3, 36-38cm] $742 \mu \mathrm{m},[64 \mathrm{R}-5,38.5-40 \mathrm{~cm}] 700 \mu \mathrm{m}$

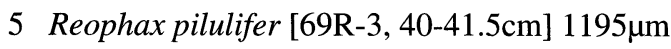

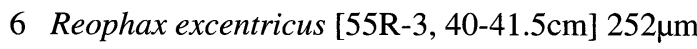

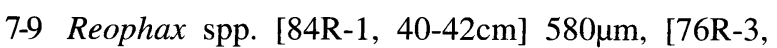
$40-41.5 \mathrm{~cm}] 721 \mu \mathrm{m},[62 \mathrm{R}-5,38-40] 959 \mu \mathrm{m}$

10 Haplophragmoides walteri [64R-5, 38.5-40] 734 $\mu \mathrm{m}$
11 Haplophragmoides walteri / carinatus [55R-1, 41.5$43 \mathrm{~cm}] 243 \mu \mathrm{m}$

12 Haplophragmoides ex gr. carinatus [60R-5, 39$40.5 \mathrm{~cm}] 540 \mu \mathrm{m}$

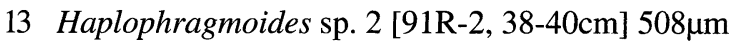

1415 Haplophragmoides ex gr. carinatus [76R-5, $40-41.5 \mathrm{~cm}] 635 \mu \mathrm{m},[76 \mathrm{R}-5,40-41.5 \mathrm{~cm}] 831 \mu \mathrm{m}$

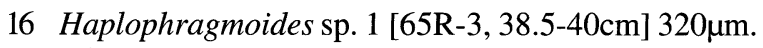
(If not labeled scale bar $=400 \mu \mathrm{m}$ ). 


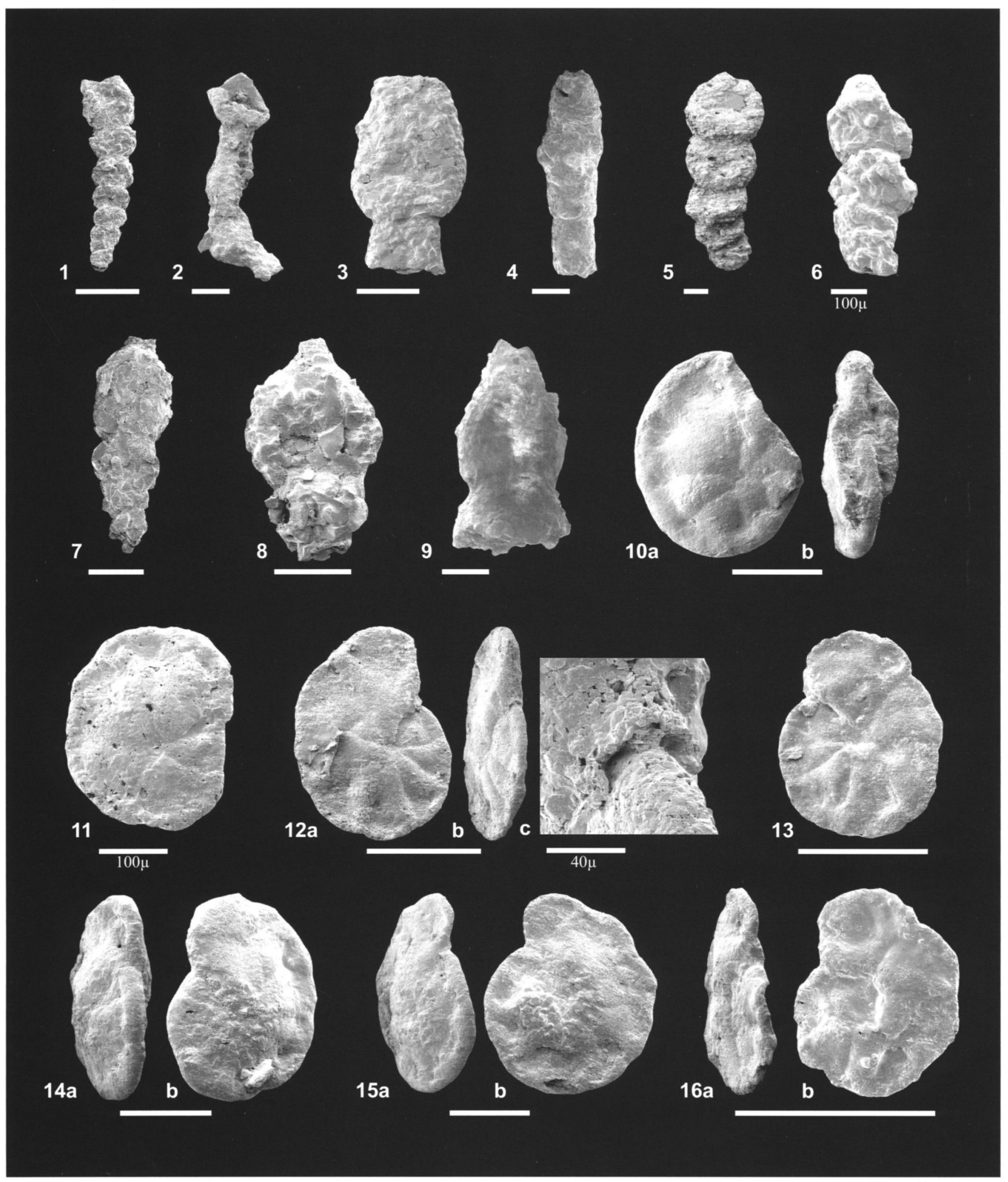


Hyperammina spp.

Various unidentifiable deformed specimens belonging to the genus Hyperammina.

Karreriella seigliei (Gradstein and Kaminski 1989)

Plate 7, figures $4,5 \mathrm{a}, \mathrm{b}, 6$

Dorothia seigliei GRADSTEIN and KAMINSKI 1989, p. 79, textfig. 3, pl. 5, figs. 1-6, pl. 6, figs. 1-5.

Karreriella seigliei (Gradstein and Kaminski 1989),-CHARNOCK and JONES 1990, p. 194, pl. 12, fig. 16, pl. 25, fig. 7.

The species appears to be endemic to the Norwegian-Greenland Sea region.

\section{Karreriella sp.}

Broken or deformed specimens closest to Karreriella seigliei.

Karrerulina horrida (Mjatliuk 1970)

Karreriella horrida MJATLIUK 1970, p. 114, pl. 4, fig. 9, pl. 33, figs $15,16 \mathrm{a}-\mathrm{b}$.

Karrerulina horrida (Mjatliuk 1970).- KAMINSKI and GRADSTEIN 2005, p. 473, pl. 117, figs 1-11.

\section{Karrerulina sp.}

Broken or deformed specimens resembling Karrerulina horrida (Mjatliuk 1970).

\section{Lagenammina sp.}

Plate 1, figures 13,14

Specimens are coarsely agglutinated.

Nothia excelsa (Grzybowski 1898)

Plate 1, figure 8

Dendrophrya excelsa GRZYBOWSKI 1898, p. 272, pl. 10, figs. 1-4. Nothia excelsa (Grzybowski).- GEROCH and KAMINSKI 1992, p. 255 , pl. 1 , figs. 1a-4, pl. 2, figs. 1-11.

Nothia latissima (Grzybowski 1898)

Dendrophrya latissima GRZYBOWSKI 1898, p. 273, pl. 10, fig. 8 .

Nothia latissima (Grzybowski).- KAMINSKI and GEROCH 1993, p. 245, pl. 1, figs. 1a-c, 14a,b.

Nothia robusta (Grzybowski 1898)

Dendrophrya robusta GRZYBOWSKI 1898, p. 273, pl. 10, fig. 7.

Nothia robusta (Grzybowski).- KAMINSKI and GRADSTEIN 2005, p. 114 , pl. 4 , figs. $1-8$ (fig. 1 is the lectotype).

\section{Nothia spp.}

Plate 1, figures 5, 6, 7, 9, 10
Unidentifiable fragments of different Nothia species.

Psamminopelta gradsteini Kaminski and Geroch 1997

Plate 2, figures 9, 10, 11

Spirosigmoilinella compressa (Matsunaga 1955).- OSTERMAN and SPIEGLER 1996, pl. 1, fig. 17.

Psamminopelta gradsteini KAMINSKI and GEROCH 1997, p. 250, textfig. 1, pl. 1, figs. 1-9.

This thin, narrow species has been previously reported under various names in the North Sea - Norwegian Sea literature. It is also found in the North Atlantic and in the Polish Carpathians (Kaminski and Geroch 1997).

\section{Psamminopelta sp.}

Plate 2, figure 12

Specimens are similar to Psamminopelta gradsteini, but are broader and less flattened.

Psammosiphonella cylindrica (Glaessner 1937)

Rhabdammina cylindrica GLAESSNER 1937, p. 354, pl. 1, fig. 1

Psammosiphonella cylindrica (Glaessner).- KAMINSKI and GRADSTEIN 2005, p. 119, pl. 5/6, figs 9-13.

Psammosiphonella discreta (Brady 1881)

Rhabdammina discreta BRADY 1881, p. 48.- BRADY 1884, p. 268, pl. 22, figs. 11-13.

Psammosiphonella discreta (Brady).- KAMINSKI and GRADSTEIN 2005, p. 117, pl. 4/5, figs 1-8.

Psammosphaera fusca Schultze 1875

Plate 1, figure 15

Psammosphaera fusca SCHULTZE 1875, p. 113, pl. 2, fig. 8a-f.

\section{Psammosphaera spp.}

Different species of Psammosphaera that are not identifiable.

Pseudonodosinella elongata (Grzybowski 1898)

Plate 3, figures 3, 4

Reophax elongata GRZYBOWSKI 1898, p. 279, pl. 10, figs. 19-20.

Pseudonodosinella elongata (Grzybowski).- KAMINSKI and GRADSTEIN 2005, p. 256.

Only fragments of this species were observed.

Pseudonodosinella nodulosa (Brady 1879)

Reophax nodulosa BRADY 1879, p. 52, pl. 4, figs 7-8.

Pseudonodosinella nodulosa (Brady).- KAMINSKI and GRADSTEIN 2005, p. 259, pl. 49, figs 1-9.

\section{PLATE 4}

1 Haplophragmoides sp. [78R-1, 40-42cm] 471 $\mu \mathrm{m}$

2 ?Ammobaculites jarvisi [94R-1, 40-41.5cm] 3369um

34 Eratidus sp. [81R-4, 39-45cm] $431 \mu \mathrm{m}$, [62R-5, $38-40 \mathrm{~cm}] 265 \mu \mathrm{m}$

5 Ammosphaeroidina sp. [69R-5, 38.5-40cm] 439 $\mu \mathrm{m}$

6 Budashevaella multicamerata [55R-1, 41.5-43cm] $650 \mu \mathrm{m}$
7 Recurvoides sp. [63R-1, 42-43.5cm] 604 $\mu \mathrm{m}$

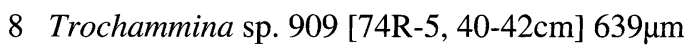

9 Trochammina lomonosovensis [60R-5, 39-40.5cm] $400 \mu \mathrm{m}$

10 Trochammina sp. [81R-4, 39-40.5cm] 546 $\mu$ m. (If not labeled scale bar $=400 \mu \mathrm{m}$ ). 


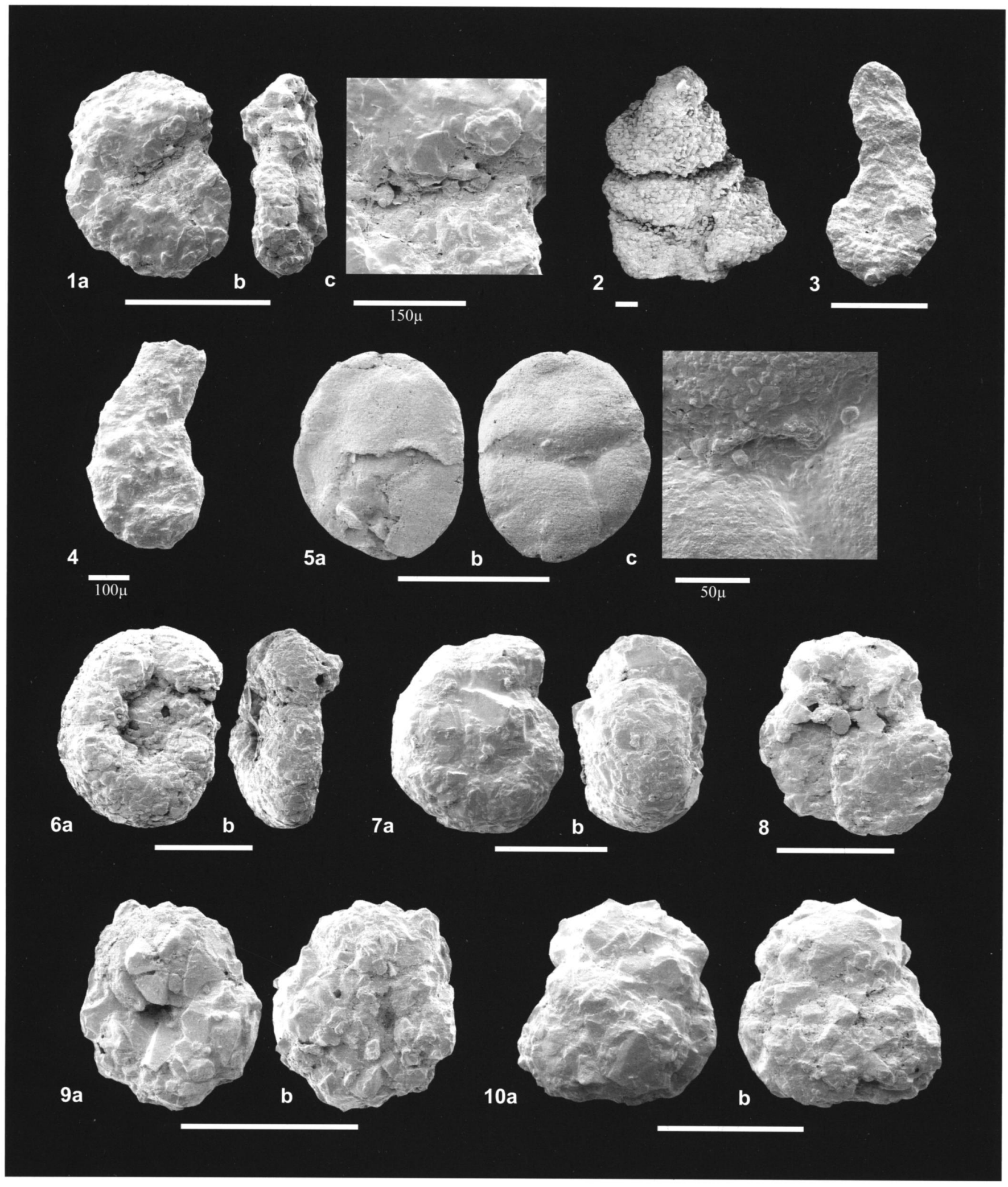




\section{Recurvoides sp.}

Plate 4, figure $7 \mathrm{a}, \mathrm{b}$.

Small streptospiral tests, relatively coarse grained, chambers increase rapidly in size.

Recurvoidella lamella (Grzybowski 1898)

Trochammina lamella GRZYBOWSKI 1898, p. 290, pl. 11, fig. 25.

Recurvoidella lamella (Grzybowski 1898).- KAMINSKI and GEROCH 1993, p. 263, pl. 10, figs. 8a-9c (figure 8 is the lectotype).

\section{Recurvoidella sp.}

Resembling broken or deformed specimens similar to Recurvoidella lamella (Grzybowski, 1898).

Reophanus berggreni Gradstein and Kaminski 1997

Plate 2, figures $16,17,18$

Hormosina carpenteri (Brady).- OSTERMAN and SPIEGLER 1996, pl. 1, fig. 11.

Reophanus berggreni GRADSTEIN and KAMINSKI 1997, p. 220, textfig. 4; fig. 5, 1-7b.

Reophax duplex Grzybowski 1896

Reophax duplex var. alpha GRZYBOWSKI 1896, p. 276, pl. 8, figs. 23-24.

Reophax excentricus Cushman 1910

Plate 3, figure 6

Reophax excentricus CUSHMAN 1910, p. 92, textfig. 134.

Reophax sp. - OSTERMAN and SPIEGLER 1996, pl. 1, fig. 7.

Reophax pilulifer Brady 1884

Plate 3, figure 5

Reophax pilulifer BRADY 1884, p. 292, pl. 30, figs 18-20.

Chambers increase in size more slowly compared with the modern species, as a result the specimens bear a resemblance to the Paleogene species Hormosina trinitatensis.

Reophax spp.

Plate 3, figures 7-9

Different broken and deformed parts of species belonging to the genus Reophax.

Repmanina charoides (Jones and Parker 1860)

Plate 2, figures 7-8

Trochammina squamata var. charoides JONES and PARKER 1860, p. 304

Glomospira charoides (Jones and Parker).- BERGGREN and KAMINSKI 1990, p. 60, pl. 1, fig. 2 (lectotype).
Reticulophragmium acutidorsatum (Hantken 1868)

Plate 6, figure 3

Haplophragmium acutidorsatum HANTKEN 1868, p. 82, pl. 1, fig. 1a,b.

Reticulophragmium acutidorsatum (Hantken).-GELLAI-NAGY 1988, pl. 1, figs. 1-3 (lectotype).

Reticulophragmium amplectens (Grzybowski 1898)

Plate 6, figure 4a,b

Cyclammina amplectens GRZYBOWSKI 1898, p. 292, pl. 12, figs. 1-3 Reticulophragmium amplectens (Grzybowski).- KAMINSKI and GEROCH 1993, p. 266, pl. 11, figs 5-7c

Reticulophragmium orbicularis (Brady 1881)

Plate 7, figure 2a,b,c

Cyclammina orbicularis BRADY 1881, p. 53.- BRADY 1884, pl. 37 , figs. 17-19.

The specimens have the tangential sutures that are typical of the modern form. This species probably forms a plexus with $R$. rotundidorsata.

Reticulophragmium pusillum (Brady 1881)

Plate 6, figures 1a,b, 2a,b

Cyclammina pusilla BRADY 1881, p. 53.

Reticulophragmium rotundidorsatum (Hantken 1875)

Plate 6, figure 7a,b, Plate 7, figure 1a,b

Haplophragmium rotindidorsatum HANTKEN 1875, p. 12, pl. 1, fig. 2. Reticulophragmium rotundidorsatum (Hantken).- HORVÁTH 2002, p. 31 , pl. 2 , fig. 2.

\section{Reticulophragmium sp. 1}

Plate 6, figure 6a,b

Deformed specimens closest to Reticulophragmium rotundidorsatum, but differing in possessing a narrower test and thin wall.

Reticulophragmium sp. 2

Deformed and broken specimens closest to Reticulophragmium amplectens.

\section{Reticulophragmium sp. 3}

Deformed and broken specimens closest to Reticulophragmium acutidorsatum.

\section{Reticulophragmium spp.}

Plate 6, figure 5a,b

Specimens belonging to this genus that remain unidentified.

\section{PLATE 5}

1-2 Trochammina spp. [85R-1, 40-41.5cm] $737 \mu \mathrm{m}$, [81R-4, 39-40.5cm] $459 \mu \mathrm{m}$

3 Conotrochammina sp. [91R-1, 38.5-40.5cm] 554 $\mu \mathrm{m}$

4 ?Karrerulina sp. fragment [73R-3,38.5-40cm] $324 \mu$
5 Veleroninoides sp. [73R-1, 38.5-40cm] $921 \mu \mathrm{m}$

6-7 Veleroninoides scitulus [60R-5, 39-40.5cm] 630 $\mu \mathrm{m}$, $[68 \mathrm{R}-1,40-41.5 \mathrm{~cm}] 956 \mu \mathrm{m}$. (If not labeled scale bar $=$ $400 \mu \mathrm{m})$. 


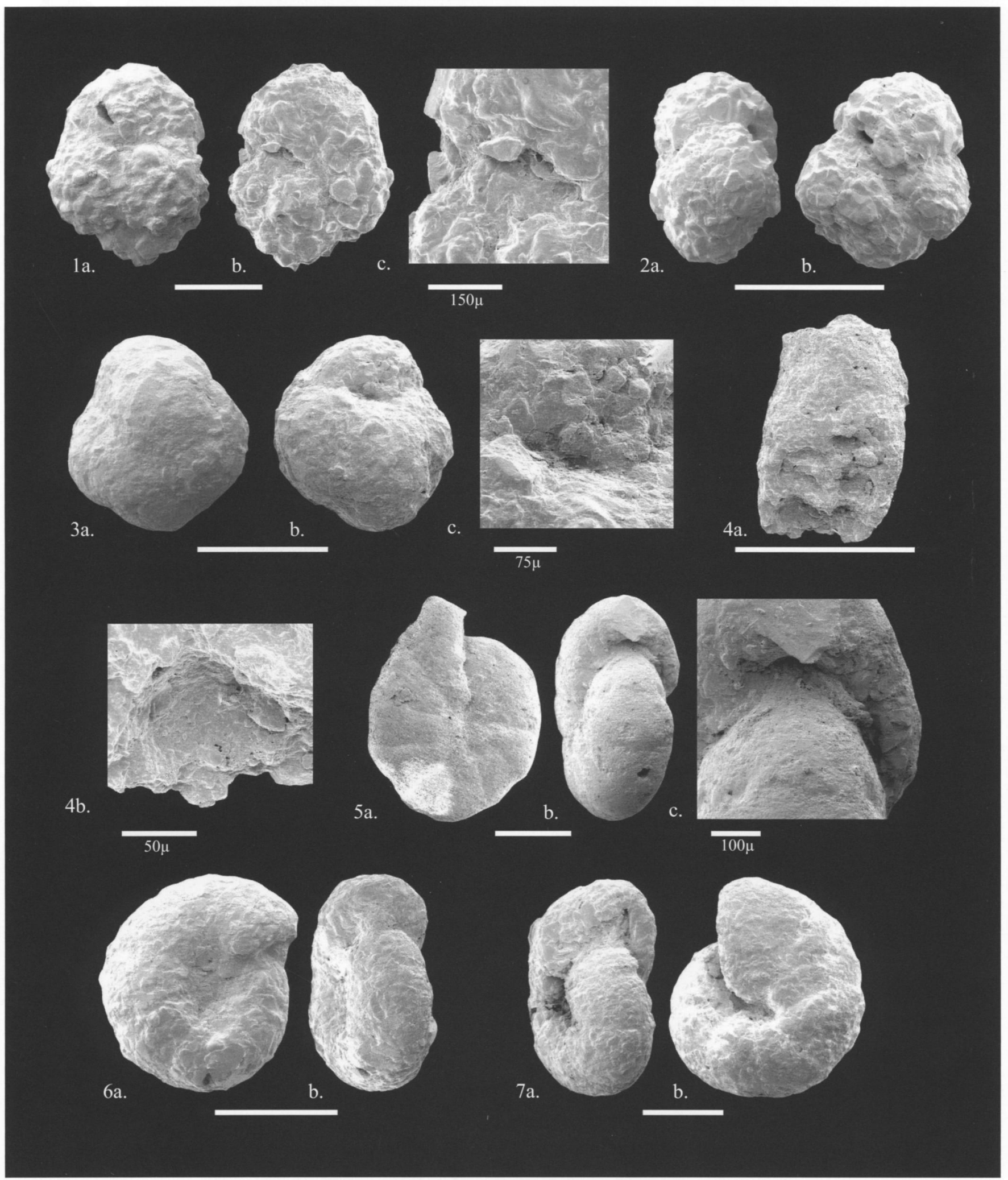


Rhabdammina abyssorum Carpenter 1869

Plate 1, figures 1,2

Rhabdammina abyssorum CARPENTER 1869, p. 288.-BRADY 1884, p. 266, pl. 21, figs 1-13.

Rhabdammina spp.

Plate 1, figure 3

Broken parts of different unidentifiable species of genus Rhabdammina.

Rhizammina spp.

Plate 1, figures 11, 12; Plate 2, figure 6

Different broken and deformed fragments of Rhizammina sp. 1 and Rhizammina sp. 2.

Saccammina cf. S. sphaerica Brady 1879

cf. Saccammina sphaerica SARS. - BRADY 1879, p. 183

Specimens differ from the modern form in their smaller dimensions and more finely agglutinated wall.

Spirosigmoilinella compressa Matsunaga 1955

Plate 2, figure 13

Spirosigmoilinella compressa MATSUNAGA 1955, p. 50, figs. 1-2. Spirosigmoilinella sp. - OSTERMAN and SPIEGLER 1996, pl. 1, fig.s 15-16.

Spirosigmoilinella sp.

Specimens resembling Spirosigmoilinella compressa Matsunaga 1955.

\section{Subreophax sp. 1}

Plate 3, figure 1

Specimens are most similar to the modern species Subreophax aduncus (Brady).

\section{Subreophax sp. 2}

Plate 3, figure 2

Specimens with more elongated, irregular chambers.

Trochammina lomonosovensis Evans and Kaminski 1998

Plate 4, figure 9a,b

Trochammina lomonosovensis EVANS and KAMINSKI 1998, p. 124, pl. 2, figs. 1-4.

\section{Trochammina spp.}

Plate 4, figures 8, 10a,b; Plate 5, figures 1a,b,c, 2a,b

Different undetermined types of genus Trochammina.
Veleroninoides scitulus (Brady 1881)

Plate 5, figures 6a,b, 7a,b

Lituola (Haplophragmium) scitulum BRADY 1881, p. 50. - BRADY 1884, pl. 34, figs. 11a-13.

Veleroninoides sp.

Plate 5, figures 5a,b,c

Specimens resembling Veleroninoides scitulus in chamber arrangement, but differing in possessing a shallower umbilicus and a more finely finished wall.

\section{REFERENCES}

ALTENBACH, A., PFLAUMANN, U., SCHIEBEL, R., THIES, A., TIMM, S., and TRAUTH, M., 1999. Scaling percentages and distributional patterns of benthic foraminifera with flux rates of organic carbon. Journal of Foraminiferal Research, 29 (3): 173-185.

BERGGREN, W.A. and KAMINSKI, M.A., 1990. Abyssal Agglutinates: Back to Basics. In: Hemleben, C., Kaminski, M.A., Kuhnt, W. and Scott, D.B., Eds., Paleoecology, Biostratigraphy, Paleoceanography and Taxonomy of Agglutinated Foraminifera, , 53-76. NATO ASI Series C327. Dordrecht: Kluwer Academic Publishers.

BERRY, E.W., 1928. The smaller foraminifera of the middle Lobitos shales of northwestern Peru. Eclogae Geologae Helvetiae, 21: $390-405$.

BOHRMANN, G., HENRICH, R., and THIEDE, J., 1990. Miocene to Quaternary paleoceanography in the northern North Atlantic: Variability in carbonate and biogenic opal accumulation. In: Bleil, U. and Thiede, J., Eds., Geological History of the Polar Oceans: Arctic vs. Antarctic, 647-675. NATO ASI Series C:308. Dordrecht: Kluwer Academic Publishers, Dordrecht.

BRADY, H.B., 1878. On the reticularian and radiolarian rhizopoda (Foraminifera and Polycystina) of the North-Polar Expedition of 1875, 1876. Annals and Magazine of Natural History, series 5, 1, 425 .

1879. Notes on some of the reticularian Rhizopoda of the "CHALLENGER" Expedition; Part I. On new or little known Arenaceous types. Quarterly Journal of Microscopical Sciences, 19: $20-67+3 \mathrm{pl}$.

1881. Notes on some of the reticularian Rhizopoda on the "Challenger" Expedition; Part III. Quarterly Journal of Microscopical Sciences, 21: 31-71.

1884. Report on the foraminifera dredged by H.M.S. CHALLENGER during the years 1873-1876. In: Murray, J., Ed., Reports of the scientific results of the voyage of the H.M.S. Challenger. Zoology, 9:1-814.

\section{PLATE 6}

1-2 Reticulophragmium pusillum [86R-1, 41-42.5cm] $1107 \mu \mathrm{m}$, [87R-1, 40-42cm] 391 $\mu \mathrm{m}$

3 Reticulophragmium acutidorsatum [65R-1, 38.5$40 \mathrm{~cm}] 594 \mu \mathrm{m}$

4 Reticulophragmium amplectens [65R-5, 38.5-40cm] $464 \mu \mathrm{m}$,
5 Reticulophragmium sp. [55R-1, 41.5-43cm] 640 $\mu \mathrm{m}$

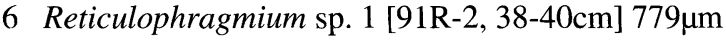

7 Reticulophragmium rotundidorsatum [93R-1, 40$41.5 \mathrm{~cm}] 529 \mu \mathrm{m}$. (If not labeled, scale bar $=400 \mu \mathrm{m}$ ). 


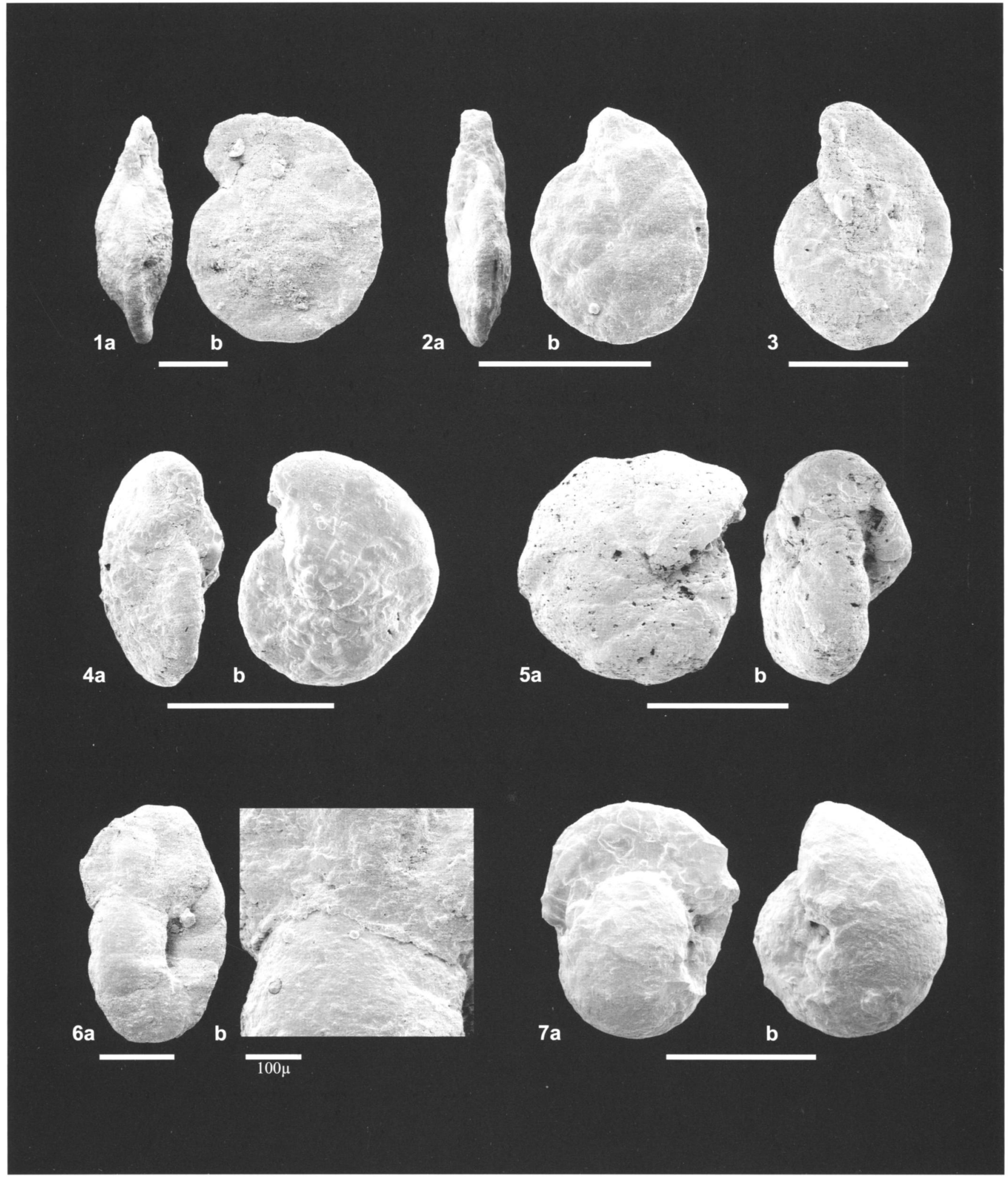


$\rightarrow$ CARPENTER, W.B., 1869. On the rhizopodal fauna of the deep sea. Proceedings of the Royal Society of London, 18: 59-62.

CHARNOCK, M.A. and JONES, R.W., 1990. Agglutinated foraminifera from the Paleogene of the North Sea. In: Hemleben, C., Kaminski, M.A., Kuhnt, W., and Scott, D.B., Eds., Paleoecology, Biostratigraphy, Paleoceanography and Taxonomy of Agglutinated Foraminifera 139-244. NATO ASI Series. Dordrecht: Kluwer Academic Publishers.

CUSHMAN, J.A., 1910. A monograph on the foraminifera of the North Pacific Ocean; Part I - Astrorhizidae and Lituolidae. United States National Museum Bulletin, 71, 134 pp.

1926. The foraminifera of the Velasco Shale of the Tampico Embayment. American Association of Petroleum Geologists Bulletin, 10:581- 612 .

CUSHMAN, J.A. and RENZ, H.H., 1941. New Oligocene-Miocene foraminifera from Venezuela. Contributions from the Cushman Laboratory for Foraminiferal Research, 17:1-27.

1946. The foraminiferal fauna of the Lizard Springs Formation of Trinidad, British West Indies. Cushman Laboratory for Foraminiferal Research Special Publication, 18: 1-48.

D'ORBIGNY, A., 1839. Foraminifères, in Ramon de la Sagra, Histoire physique, politique et naturelle de l'île de Cuba. Paris: Arthus Bertrand.

$\rightarrow$ EVANS, J.R. and KAMINSKI, M.A., 1998. Pliocene and Pleistocene chronostratigraphy and paleoenvironment of the central Arctic Ocean, using deep-water agglutinated foraminifera. Micropaleontology, 44:109-130.

GELLAI-NAGY, A., 1988. Delineation of Hantken's foraminiferal species from the original collection. A Magyar Állami Földtani Intézet Évi Jelentése 1988-évrõl, II Rész, 133-173.

GEROCH, S. and KAMINSKI, M.A., 1992. The morphology and systematics of Nothia excelsa (Grzybowski), a deep-water agglutinated foraminifer. Rocznik Polskiego Towarzystwa Geologicznego, 62: $255-265$.

GLAESSNER, M.F., 1937. Studien über Foraminiferen aus der Kreide und dem Tertiär des Kaukasus; 1. Die Foraminiferen der ältesten Tertiärschichten des Nordwest-Kaukas. Problems of Paleontology, 2-3: 349-408. Moscow.

GRADSTEIN, F.M. and BERGGREN, W.A., 1981. Flysch-type agglutinated foraminifera and the Maestrichtian to Paleogene history of the Labrador and North Seas. Marine Micropaleontology, 6: 211-268. $\rightarrow$ GRADSTEIN, F.M. and KAMINSKI, M.A., 1989. Taxonomy and biostratigraphy of new and emended species of Cenozoic deep-water agglutinated foraminifera from the Labrador and North Seas. Micropaleontology, 35: 72-92.

1997. New species of Paleogene deep-water agglutinated foraminifera from the North Sea and Norwegian Sea. Annales Societatis Geologorum Poloniae, 67: 217-229.

GRADSTEIN, F.M., KAMINSKI, M.A., BERGGREN, W.A. and D'IORIO, M.A., 1994. Cenozoic biostratigraphy of the Central North Sea and Labrador Shelf. Micropaleontology vol. 40 Supplement, $152 \mathrm{pp}$.

GREEN, R.C., KAMINSKI, M.A., and SIKORA, P.J., 2004. Miocene deep water agglutinated foraminifera from Viosca Knoll, offshore Louisiana (Gulf of Mexico). In: Bubík, M., and Kaminski, M.A., Eds). Proceedings of the Sixth International Workshop on Agglutinated Foraminifera, 119-144. Grzybowski Foundation Special Publication no. 8 .

GRZYBOWSKI, J., 1896. Otwornice czerwonych ilów z Wadowic. Rozprawy Wydzialu Matematyczno-Przyrodniczego, Akademia Umiejetnosci w Krakowie, serya 2, 30: 261-308.

1898. Otwornice pokladów naftonosnych okolicy Krosna. Rozprawy Wydzialu Matematyczno-Przyrodniczego, Akademia Umiejetnosci w Krakowie, serya 2, 33: 257-305.

1901. Otwornice warstw inoceramowych okolicy Gorlic. Rozprawy Wydzialu Matematyczno-Przyrodniczego, Akademia Umiejetnosci w Krakowie, serya 2, 41: 219-286.

HANTKEN, M., von, 1868. A kis-czelli tályag foraminiferái. Magyar Földtani Társulat Munkálatai, Pest, 4: 75-96.

1875. Die Fauna der Clavulina szaboi-Schichen; Theil I Foraminiferen. Mittheilungen aus dem Jahrbuche der Königlich-Ungarische Geologische Anstalt, Budapest, 4 (1):1-93 + 13 pls.

HORVÁTH, M. 2002. Data to revision and distribution of small Foraminifera species described by Hantken $(1868,1875)$. Part 1. Textulariidae and Miliolidae. Fragmenta Palaeontologica Hungarica, 20: 25-42.

HULL, D.M., OSTERMAN, L. and THIEDE, J., 1996. Biostratigraphic synthesis of Leg 151, North Atlantic-Arctic Gateways. Proceedings of the Ocean Drilling Program, Scientific Results, 151: 627-644.

JONES, R.W. and CHARNOCK, M.A., 1985. "Morphogroups" of agglutinating foraminifera. Their life positions and feeding habits and potential applicability in (paleo)ecological studies. Revue de Paleobiologie, 4: 311-320.

\section{PLATE 7}

1 Reticulophragmium rotundidorsatum [65R-1, 38.5$40 \mathrm{~cm}] 414 \mu \mathrm{m}$

2 Reticulophragmium orbicularis [66R-1, 38.5-40cm] $947 \mu \mathrm{m}$
3 Cyclammina sp. [82R-1, 40-41.5cm] 1668 $\mu \mathrm{m}$

4-6 Karreriella siegliei 4, [94R-3, 35-36.5cm] 484 $\mu \mathrm{m} ; 5$,

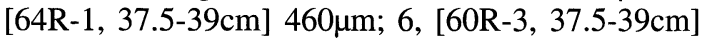
$928 \mu \mathrm{m}$. (If not labeled, scale bar $=400 \mu \mathrm{m}$ ). 


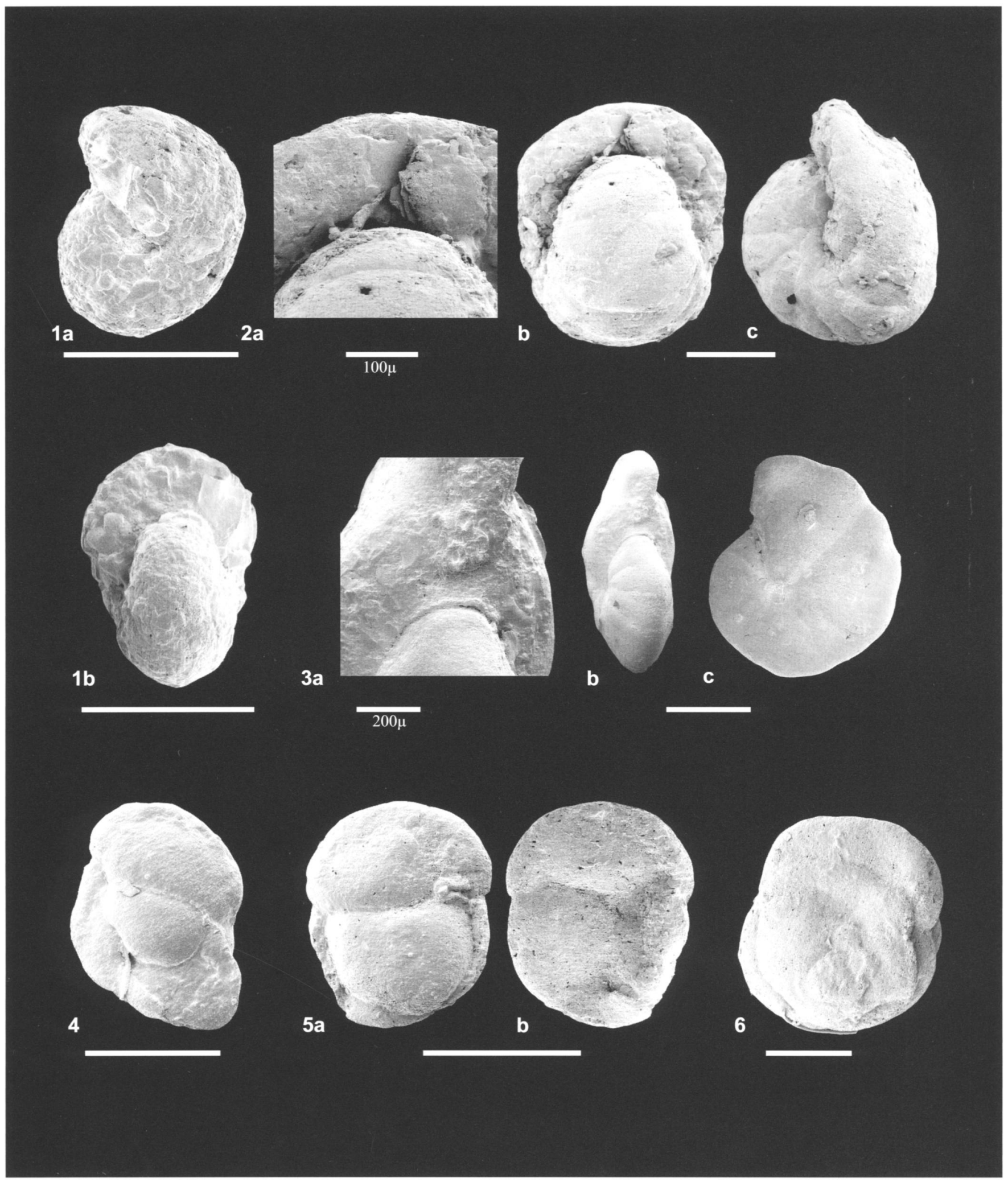


JONES, T.R. and PARKER, W.K. 1860. On the Rhizopodal fauna of the Mediterranean compared with that of the Italian and some other Tertiary deposits. Quarterly Journal of the Geological Society of London, 16: 292-307.

KAMINSKI, M.A. and AUSTIN, W.E.N., 1999. Oligocene deep-water agglutinated foraminifers at Site 985, Norwegian Basin, southern Norwegian Sea. Proceedings of the Ocean Drilling Program, Scientific Results, 162: 169-177.

KAMINSKI, M.A. and GEROCH, S., 1993. A revision of foraminiferal species in the Grzybowski Collection. In: Kaminski, M.A., Geroch, S. and Kaminski, D., Eds., The Origins of Applied Micropaleontology: The School of Jozef Grzybowski. Grzybowski Foundation Special Publication, 1: 239-323.

1997. Psamminopelta gradsteini n.sp., a new species of Paleogene deep-water agglutinated foraminifera from the northern North Atlantic and Polish Outer Carpathians. In: Hass, H.C. and Kaminski, M.A., Eds., Contributions to the Micropaleontology and Paleoceanography of the Northern North Atlantic, 249-252. Grzybowski Foundation Special Publication no. 5.

KAMINSKI, M.A. and GRADSTEIN, F.M., 2005. Atlas of Paleogene Cosmopolitan deep-water Agglutinated Foraminifera. Grzybowski Foundation Special Publication, 10: 547 pp.

KAMINSKI, M.A., GRADSTEIN, F.M., BERGGREN, W.A., GEROCH, S. and BECKMANN, J.P., 1988. Flysch-type agglutinated foraminiferal assemblages from Trinidad: Taxonomy, Stratigraphy and Paleobathymetry. Abhandlungen der Geologishen Bundesanstalt, 41: 155-228.

KAMINSKI, M.A., GRADSTEIN, F.M., SCOTT, D.B. and MacKINNON K.D., 1989. Neogene benthic foraminiferal stratigraphy and deep water history of Sites 645, 646 and 647, Baffin Bay and Labrador Sea. Proceedings of the Ocean Drilling Program, Scientific Results, 105: 705-730.

KAMINSKI, M.A., GRADSTEIN, F.M., GOLL, R.M. and GRIEG, D., 1990. Biostratigraphy and paleoecology of deep-water agglutinated foraminifera at ODP Ste 643, Norwegian-Greenland Sea. In: Hemleben, C., Kaminski, M.A., Kuhnt, W., and Scott, D.B., Eds., Paleoecology, Biostratigraphy, Paleoceanography and Taxonomy of Agglutinated Foraminifera, 345-386. NATO ASI Series. Dordrecht: Kluwer Academic Publishers.

KNIES, J. and MANN, U., 2002. Depositional environment and source rock potential of Miocene strata from the central Fram Strait: introduction of a new computing tool for simulating organic facies variations. Marine and Petroleum Geology, 19: 811-828.

KRISTOFFERSON, Y., 1990. On the tectonic evolution and paleoceanographic significance of the Fram Strait Gateway. In: Bleil, U. and Thiede, J., Eds., Geological History of the Polar Oceans: Arctic vs. Antarctic, NATO ASI Series C:308, pp. 63-76. Dordrecht: Kluwer Academic Publishers.

MANUM, S., BOUTER, M.C., GUNNARSDOTTIR, H., RANGNES, K., and SCHOLZE, A., 1989. Eocene to Miocene palynology of the Norwegian-Greenland Sea (ODP Leg 104). Proceedings of the Ocean Drilling Program, Scientific Results, 151:611-662.

MATSUNAGA, T., 1955. Spirosigmoilinella, a new foraminiferal genus from the Miocene of Japan. Transactions and Proceedings of the Palaeontological Society of Japan, n.s., Tokyo, 18: 49-50.

MCNEIL, D.H., 1990. Tertiary marine events of the Beaufort-MacKenzie Basin and correlation of Oligocene to Pliocene marine outcrops in Arctic North America. Arctic, 43 (4): 301-313.
MJATLIUK, E.V., 1966. K voprosu o foraminiferakh c kremnevnym skeletom [On the question of foraminifera with a siliceous skeleton]. Voprosy Mikropaleontologii, 10:255-269.

1970. Foraminifery flishevykh otlozhenii vostochnykh Karpat (Mel-Paleogen) [Foraminifera of the flysch deposits of the eastern Carpathians]. Trudy Vsesoyuznogo Nauchno-Issledovatel'skogo Geologorazvedochnogo Instituta VNIGRI, 282, 1-225. Leningrad.

$\rightarrow$ MILLER, K.G., GRADSTEIN, F.M. and BERGGREN, W.A., 1982. Late Cretaceous to early Tertiary agglutinated benthic foraminifera in the Labrador Sea. Micropaleontology, 28(1): 1-30.

MYHRE, A.M. and THIEDE, J., 1995. North Atlantic-Arctic Gateways. Proceedings of the Ocean Drilling Program, Initial Reports, 151: 5-26.

NAGY, J., 1992. Environmental significance of foraminiferal morphogroups in Jurassic North Sea deltas. Paleogeography, Paleoclimatology, Paleoecology, 95: 111-134.

NAGY, J. GRADSTEIN, F.M., KAMINSKI, M.A., HOLBOURN, A.E.L., 1995. Foraminiferal morphogroups, paleoenvironments and new taxa from Jurassic and Cretaceous strata of Thakkhola, Nepal. In: Kaminski, M.A., Geroch, S., and Gasinski, MA, Eds., Proceedings of the Fourth International Workshop on Agglutinated Foraminifera, 181-209. Grzybowski Foundation Special Publication no. 3 .

NAGY, J., KAMINSKI, M.A., JOHNSEN, K., and MITLEHNER, A.G., 1997. Foraminiferal, palynomorph, and diatom biostratigraphy and paleoenvironments of the Torsk Formation: A reference section for the Paleocene-Eocene transition in the western Barents Sea. In: Hass, H.C. and Kaminski, M.A. Contributions to the Micropaleontology and Paleoceanography of the Northern North Atlantic, 15-38. Grzybowski Foundation Special Publication no. 5.

NAGY, J., KAMINSKI, M.A., KUHNT, W., and BREMER, M.A., 2000. Agglutinated foraminifera from neritic to bathyal facies in the Palaeogene of Spitsbergen and the Barents Sea. In: Hart, M.B., Kaminski, M.A. and Smart, C.W., Eds., Proceedings of the Fifth International Workshop on Agglutinated Foraminifera, 333-361. Grzybowski Foundation Special Publication no. 7.

NAGY, J., KAMINSKI, M.A., GRADSTEIN, F.M., and JOHNSON, K., 2004. Quantitative foraminiferal and palynomorph biostratigraphy of the Paleogene in the southwestern Barents Sea. In: Bubik, M. and Kaminski, M.A., Eds., Proceedings of the Sixth International Workshop on Agglutinated Foraminifera, 359-379. Grzybowski Foundation Special Publication no. 8.

O'CONNELL, S., WOLF-WELLING, T.C.W., CREMER, M. and STEIN, R., 1996. Neogene paleoceanography and paleoclimatic history from Fram Strait: changes in accumulation rates. Proceedings of the Ocean Drilling Program, Scientific Results, 151:569-578.

OSTERMAN, L. and SPIEGLER, D., 1996. Agglutinated benthic foraminiferal biostratigraphy of Ocean Drilling Program (ODP) Sites 909 and 913, northern North Atlantic. Proceedings of the Ocean Drilling Program, Scientific Results, 151:169-185.

POULSEN, N.E., MANUM, S.B., WILLIAMS, G.L., and ELLEGAARD, M., 1996. Tertiary dinoflagellate biostratigraphy of Sites 907, 908, and 909 in the Norwegian-Greenland Sea. Proceedings of the Ocean Drilling Program, Scientific Results, 151: 255-287.

PREECE, R.C., KAMINSKI, M.A. and DIGNES, T.W., 1999. Miocene benthic foraminiferal morphogroups in an oxygen minimum zone, offshore Cabinda. In: Cameron, N.R., Bate, R.H., and Clure, V.S., Eds., The oil and gas habitats of the South Atlantic, 267-282. Geological Society Special Publication no. 153 
REUSS, A.E., 1851. Über die fossilen Foraminiferen und Entomostraceen der Septarianthone der Umgegend von Berlin. Deutche Geologische Gesellschaft, Zeitschrift, Berlin, 3: 49-91.

ROBERTSON, D., 1891. Trochammina bradyi n.n. Annals and Magazine of Natural History, ser. 6, 7: 388.

SCHRÖDER-ADAMS, C.J. and MCNEIL, D.H., 1994. Oligocene to Miocene agglutinated foraminifera in deltaic and deep-water facies of the Beaufort-MacKenzie Basin. Geological Survey of Canada Bulletin, 477, $67 \mathrm{pp}$.

SCHULTZE, F.E., 1875. Zoologische Ergebnisse der Nordseefart vom 21 Juli bis 9 September 1872; 1. Rhizopoden. Commission der Wissenschaftliche Untersuchung Deutschlands Meere von Kiel, Jahresberichte. Berlin, Deutschland, 1875, Jahrg. 2-3.

SHIPBOARD SCIENTIFIC PARTY, 1995. Site 909. Proceedings of the Ocean Drilling Program, Initial Reports, 151:159-220.

SMITH, W.O. and NELSON, D., 1986. Importance of ice edge phytoplankton production in the Southern Ocean. BioScience, 36: 251-257.

SPIEGLER, D., 1996. Planktonic foraminifer Cenozoic biostratigraphy of the Arctic Ocean, from Fram Strait (Sites 908-909), Yermak Plateau (Sites 910-912), and East Greenland Margin (Site 913). Proceedings of the Ocean Drilling Program, Scientific Results, 151: $153-167$

STEIN, R., and STAX, R., 1996. Organic carbon and n-alkane distribution in late Cenozoic sediments of Arctic Gateways Sites 909 and 911 and their paleoenvironmental implications: preliminary results. Proceedings of the Ocean Drilling Program, Scientific Results, 151: 391-405.

THIEDE, J. and MYHRE, A.M., 1996. Introduction to the North Atlantic - Arctic Gateways: plate tectonic-paleogeographic history and significance. Proceedings of the Ocean Drilling Program, Scientific Results, 151: 2-23

VAN DEN AKKER, T.J.H.A., KAMINSKI, M.A., GRADSTEIN, F.M. and WOOD, J., 2000. Campanian to Palaeocene biostratigraphy and palaeoenvironments in the Foula Sub-basin, west of the Shetland Islands, UK. Journal of Micropalaeontology, 19 (1): 23-43.

VERDENIUS, J.G. and VAN HINTE, J.E., 1983. Central Norwegian-Greenland Sea: Tertiary arenaceous foraminifera, biostratigraphy and environment. Proceedings of the First Workshop Arenenaceous Foraminifera, 7-9 Sept. 1981. Continental Shelf Institute Publication, 108, 173-224.

VOLOSHINOVA, N.A. and BUDASHEVA, A.I., 1961. Lituolids and trochamminids from the Tertiary deposits of Sakhalin Island and the Kamchatka Peninsula (in Russian). In: Microfauna of the USSR, Trudy Vsesoyuznogo Nauchno-Issledovatel'skogo Geologorazvedochnogo Instituta VNIGRI, 170, 170-272. Leningrad.

WINKLER, A. WOLF-WELLING, T.C.W., STATTEGGER, K. and THIEDE, J., 2002. Clay mineral sedimentation in high northern latitude deep-sea basins since the Middle Miocene (ODP Leg 151, NAAG). International Journal of Earth Sciences, 91:133-198.

WOLF-WELLING, T.C.W., CREMER, M., O'CONNELL, S., WINKLER, A., and THIEDE, J., 1996. Cenozoic Arctic gateway paleoclimate variability: Indications from changes in coarse-fraction composition. Proceedings of the Ocean Drilling Program, Scientific Results, 151: 515-567.

WOLLENBURG, J.E. and MACKENSEN, A., 1998. Living benthic foraminifers from the central Arctic Ocean: faunal composition, standing stock, and diversity. Marine Micropaleontology, 34: 153-185.

Manuscript received April 18, 2005

Manuscript accepted December 14, 2005 
APPENDIX 1

ODP Hole 909 data.

\begin{tabular}{|c|c|c|c|c|c|c|c|c|c|c|c|c|c|c|c|c|c|c|c|c|c|c|c|c|c|c|c|c|c|c|c|}
\hline $\begin{array}{l}\text { Core, section, } \\
\text { interval (cm) }\end{array}$ & $\begin{array}{c}\text { Depth } \\
\text { (mbsf) }\end{array}$ & 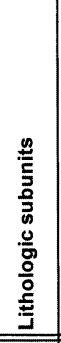 & 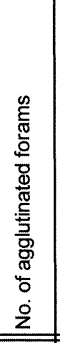 & 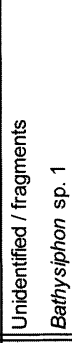 & 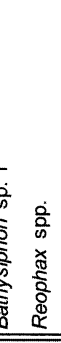 & 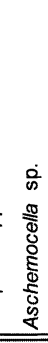 & 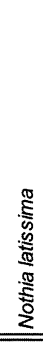 & & 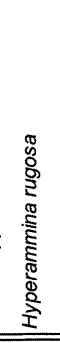 & 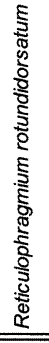 & 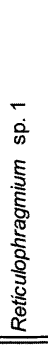 & 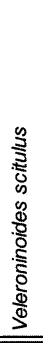 & 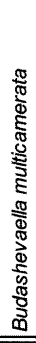 & 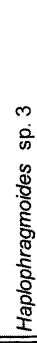 & 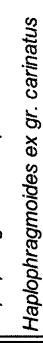 & 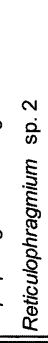 & 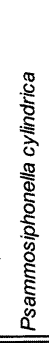 & 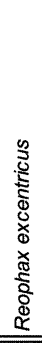 & 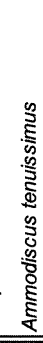 & 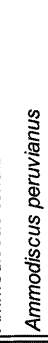 & 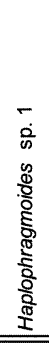 & 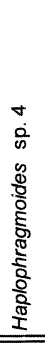 & 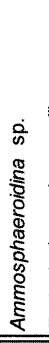 & 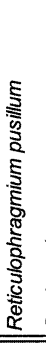 & 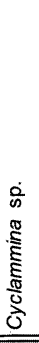 & 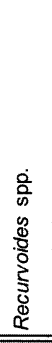 & 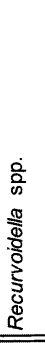 & 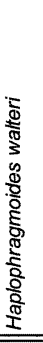 & & 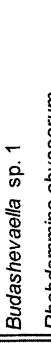 & 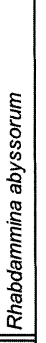 \\
\hline \multicolumn{32}{|l|}{ 151-909C- } \\
\hline $51 R-1,37-39$ & 6.97 & & 0 & . & . & & . & . & . & . & & & . & & & & & & & · & . & & & & & & & & & & \\
\hline $51 \mathrm{R}-3,38.5-40$ & 69.985 & & 0 & . & . & & . & . & . & . & . & . & 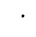 & . & . & . & 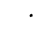 & & & . & . & & & & & & - & & & & \\
\hline 51R-5, 35-37 & 572.95 & & 0 & . & . & . & . & . & . & . & . & . & . & . & . & . & . & . & & . & . & & . & & & & . & & & & \\
\hline $52 \mathrm{R}-1,38.5-40$ & 576.585 & & 0 & . & . & . & . & . & . & . & . & . & . & . & . & . & . & . & & . & . & & . & & & . & . & & & & \\
\hline $52 R-3,38.5-40$ & 579.585 & & 0 & . & . & . & . & . & . & . & . & . & . & . & . & . & . & . & & . & . & & . & & & ${ }^{\circ}$ & . & & & & \\
\hline $52 R-4,35.5-37$ & 581.055 & & 0 & . & . & . & . & . & . & . & . & . & . & . & . & . & . & . & & . & . & & . & . & & . & . & & & & \\
\hline $53 R-1,37.5-39.5$ & 586.175 & & 0 & . & . & . & . & . & . & . & . & . & . & . & . & . & . & & & . & . & & . & . & & 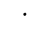 & . & & & & \\
\hline $53 R-3,38-40$ & 589.18 & & 1 & 1 & . & . & . & . & . & . & . & . & . & . & & . & . & . & . & . & . & & . & . & . & . & . & & & & \\
\hline $53 R-5,38.5-40$ & 592.185 & & 0 & . & . & . & . & . & . & . & . & . & . & . & & . & . & 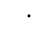 & & . & . & & . & . & & . & . & & & & \\
\hline $54 R-1,37-38.5$ & 595.87 & & 0 & . & . & . & . & . & . & . & . & & . & . & & . & . & . & . & . & . & & . & . & & . & . & & & & \\
\hline $54 R-2,38-40$ & 597.38 & & 0 & . & . & . & . & . & . & . & . & - & . & . & & . & . & . & & . & . & & . & . & & . & . & & & 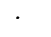 & \\
\hline $54 R-3,37-39$ & 598.87 & & 3 & & 2 & 1 & . & . & . & . & & . & . & . & & . & . & . & . & . & . & & . & . & . & . & . & . & & . & \\
\hline $55 \mathrm{R}-1,41.5-43$ & 605.515 & & 96 & . & . & . & 6 & 16 & 613 & 10 & 3 & 3 & 2 & 1 & 38 & 84 & . & . & & . & . & & . & . & & . & . & & & . & \\
\hline $55 R-3,40-41.5$ & 608.5 & & 87 & . & . & . & . & . & . & 25 & 1 & 1 & . & 5 & 18 & & 6 & 1 & 3 & 2 & 1 & 8 & 2 & 6 & 1 & 7 & & . & & . & \\
\hline $55 R-5,38-39.5$ & 611.48 & & 3 & . & . & . & . & 1 & . & · & . & . & . & . & . & . & . & . & . & . & . & & . & . & & 2 & & & & . & \\
\hline $56 \mathrm{R}-1,43-45$ & 615.23 & & 0 & & . & . & . & . & . & . & . & . & . & . & . & . & . & . & . & . & . & . & . & . & . & & & & & 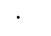 & \\
\hline $57 R-1,37.5-39$ & 624.775 & & 4 & . & . & . & . & . & . & . & 3 & . & . & . & . & . & . & - & . & & . & . & . & & & & 1 & & & . & \\
\hline $57 R-3,39-40.5$ & 627.79 & & 0 & . & . & . & . & . & . & . & . & . & . & . & . & . & . & . & . & . & . & . & . & . & & . & & & 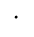 & . & \\
\hline $57 R-5,37-38.5$ & 630.77 & & 0 & . & . & . & . & . & . & . & . & . & . & . & . & . & . & . & . & . & . & . & . & . & & & . & & & . & \\
\hline $58 R-1,38.5-40$ & 634.485 & & 0 & & . & . & . & . & . & . & . & . & . & . & . & . & . & . & . & . & . & . & . & & & . & . & & & . & \\
\hline $58 R-2,38.5-40$ & 635.985 & IIIF & 0 & & . & . & . & . & . & . & . & . & . & . & . & . & . & . & . & . & . & . & . & . & & . & . & & & . & \\
\hline $59 \mathrm{R}-1,38.5-40$ & 644.085 & & 0 & & . & . & . & . & . & . & . & . & . & & . & . & . & & & . & . & & . & & & . & . & & & & \\
\hline $59 \mathrm{R}-3,38.5-40$ & 647.085 & & 54 & & . & . & 2 & 6 & 1 & 9 & 10 & . & . & & 2 & . & . & . & & . & 2 & & . & 5 & & & 8 & 4 & & 1 & \\
\hline 59R-5, 37.5-39 & 650.075 & & 107 & & . & . & . & 7 & 1 & 24 & 23 & 3 & & 4 & 10 & 0 . & . & . & 3 & . & . & & 1 & 5 & & 5 & & r & & 1 & \\
\hline $60 R-1,38.5-40$ & 653.785 & & 47 & & . & . & . & 1 & 1 & 2 & 2 & . & . & 15 & & .. & . & . & 1 & . & 1 & 2 & & & & 1 & . & & 16 & & \\
\hline 60R-3, 37.5-39 & 656.775 & & 127 & . & . & . & 3 & 14 & 48 & 20 & 10 & & . & . & 4 & . & & . & 4 & 3 & 2 & & 9 & 19 & & 5 & 1 & 7 & & & \\
\hline $60 R-5,39-40.5$ & 659.79 & & 109 & & 1 & . & & 6 & 1 & 16 & 24 & 5 & 1 & & 15 & $\begin{array}{ll}5 & 1\end{array}$ & . & . & 2 & . & . & & 4 & 12 & & 3 & . & & & 2 & \\
\hline $61 \mathrm{R}-1,38.5-40$ & 663.385 & & 1 & . & . & . & . & . & . & 1 & . & . & . & . & . & .. & . & . & . & . & . & . & . & & & . & . & . & . & & \\
\hline $61 R-3,36-38$ & 666.36 & & 169 & & 1 & & 1 & 13 & 32 & 4 & 52 & 9 & & 3 & 12 & 21 & . & & 3 & 1 & . & 2 & 1 & 2 & & 10 & 1 & 4 & 8 & & 4 \\
\hline $61 R-5,37.5-39$ & 669.375 & & 58 & . & . & . & . & 1 & . & 3 & 3 & 1 & 1 & 12 & & .. & . & . & . & . & 3 & & 5 & & & 2 & . & . & 10 & & \\
\hline $62 R-1,38-40$ & 673.08 & & 1 & & . & . & . & & . & . & 1 & . & . & & . & . & . & . & . & . & . & . & . & . & & & . & & & & \\
\hline $62 R-3,38.5-40$ & 676.085 & & 0 & & . & . & . & . & . & . & . & . & . & . & . & . & . & . & . & . & . & . & . & & & . & . & & & & \\
\hline$R-5,38-40$ & 679.08 & & 156 & & $4 \quad 14$ & . & . & 8 & . & 2 & 13 & & & 13 & $\begin{array}{ll}3 \quad 3 \\
\end{array}$ & 3. & . & & 4 & 1 & 15 & & 4 & 3 & & 9 & & 1 & 22 & 1 & \\
\hline $63 R-1,42-43.5$ & 682.72 & & 73 & & 3 & 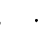 & 5 & 3 & & 2 & 5 & . & & 1 & 13 & 3 . & & . & . & 1 & . & & 9 & & & 12 & 1 & & 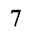 & & \\
\hline R-3, 43-44.5 & 68 & & 6 & & & . & . & 2 & & 8 & 16 & . & & 5 & 6 & 5. & . & . & 1 & . & . & & 3 & & & 3 & 2 & 3 & & 1 & \\
\hline $63 R-4,37.5-39$ & 687.175 & & 15 & & 1 & . & . & 3 & . & 3 & 18 & 1 & 2 & & 9 & . & & . & 2 & . & 1 & 1 & 12 & 12 & & 7 & 12 & 6 & 22 & & \\
\hline Q-1, 37.5-39 & 2.375 & & & & 1 & . & . & 6 & & 9 & 16 & & & & . & . & & . & 1 & . & 3 & 5 & 3 & & & 16 & 8 & 2 & & 2 & \\
\hline $64 R-3,40-41.5$ & 695.4 & & 12 & & 2 & . & . & 2 & 1 & 21 & 29 & 5 & & & 2 & . & & . & 4 & . & . & 4 & 3 & 6 & & 9 & & 6 & 1 & & \\
\hline $64 R-5,38.5-40$ & 8.385 & & 132 & & 6 & . & 2 & 24 & & 20 & 9 & . & 2 & 1 & 9 & . & & . & 1 & 1 & . & 3 & . & 20 & & 5 & 5 & 6 & 3 & 1 & \\
\hline $65 R-1,38.5-40$ & 701.985 & & 69 & & 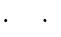 & . & 2 & 3 & & 8 & 15 & . & 4 & & & . & & . & . & . & . & 3 & . & & & 6 & 4 & $\mathrm{~s}$ & . & 4 & \\
\hline $65 R-3,38.5-4 C$ & 4.985 & & 114 & & . & & 1 & & & 6 & 42 & 4 & & 2 & 1 & . & & & . & . & 2 & & 7 & 14 & & 12 & 3 & 2 & 2 & 2 & \\
\hline $65 \mathrm{R}-5,38.5-40$ & 707.985 & & 7 & & . & . & 1 & 10 & & 6 & 8 & . & 1 & 0 & & . & & . & . & . & . & 1 & 4 & & & 4 & 7 & 4 & & & \\
\hline$R-1,38.5-40$ & 1.485 & & 146 & & . & . & & 15 & & 31 & 12 & & & 11 & 124 & & & & & . & 3 & 1 & · & 8 & & 14 & 2 & & 8 & 2 & \\
\hline $66 \mathrm{R}-2,36.5-38$ & 712.965 & & 8 & & 1 & & . & 6 & 2 & & 9 & . & . & & 2 & & & . & . & . & 5 & 5 & 11 & 5 & & 5 & 2 & & & & \\
\hline $67 \mathrm{R}-1,40-41.5$ & 721.2 & & 16 & & 1 & . & . & 2 & & 37 & 16 & & : & 4 & 13 & & & 1 & & . & . & . & 9 & 14 & & 9 & 6 & 4 & 6 & 1 & \\
\hline $\mathrm{R}-3,40-41.5$ & & & 12 & . & 2 & . & 1 & 5 & 3 & & & & . & 1 & 10 & & & & . & . & 3 & . & . & . & & . & & 5 & & 1 & \\
\hline $67 R-5,39-40.5$ & 727.19 & & 155 & & & & & 6 & & & 55 & & . & 4 & - 8 & & & & 2 & ? & & 3 & . & 18 & & 10 & & 5 & 16 & & \\
\hline $68 R-1,40-41.5$ & & & 137 & . & . & & & 1 & 1 & 15 & 30 & 1 & & 1 & 20 & & & & . & . & 7 & & . & 17 & 1 & 8 & 9 & & & 2 & \\
\hline $68 R-3,40-41.5$ & & & 8 & & . & & 1 & 3 & 1 & & 16 & 1 & & 4 & & & & & & & & 2 & 5 & 9 & & . & & 3 & & 1 & \\
\hline $2-4,38.5-4 c$ & & & 8 & & 1 & & 1 & 3 & & 4 & 4 & & . & 1 & 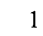 & & & 2 & . & . & & & 10 & 18 & & . & 5 & 5 & & & \\
\hline $69 \mathrm{R}-1,38.5-40$ & 740.385 & & 62 & & 1 & & & 6 & . & & 2 & 1 & & 3 & 1 & & & & & . & 8 & 10 & 4 & 6 & & 3 & 5 & 1 & 5 & . & \\
\hline $69 \mathrm{R}-3,40-41.5$ & & & 75 & 5 & . & & 3 & 5 & 1 & 2 & 9 & & 1 & & & & & . & . & . & 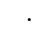 & & . & 2 & . & . & . & & 11 & 1 & \\
\hline $69 R-5,38.5-40$ & 746.385 & & 111 & 1 & 1 & & 1 & 9 & 4 & & 8 & & & & & & & . & . & & & 3 & 5 & 1 & & 2 & 1 & 2 & & . & \\
\hline $70 \mathrm{R}-1,38.5-40$ & 74 & & 110 & & 5 & & & 7 & 2 & 17 & 4 & 2 & & & & & & . & . & & & 5 & 7 & 14 & & 7 & & 2 & & . & \\
\hline 2-3, 37.5-39.5 & 752.97 & & 91 & . & · 11 & & t & 7 & 1 & & 3 & & & 14 & & & & & & & & & 2 & 7 & 2 & 4 & 3 & & & 3 & \\
\hline $70 R-5,42.5-44$ & 75 & & 88 & . & .. & & 1 & 10 & 1 & 5 & 4 & 1 & 1 & 3 & & 1 & & & 2 & & 2 & 8 & 2 & 1 & & 6 & 3 & 5 & & 1 & \\
\hline 7 & 759.685 & & 103 & & 3 & 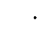 & & 7 & 2 & 3 & 2 & & & & & & & & & & & 8 & 6 & 3 & & 10 & 2 & & 12 & 3 & \\
\hline $71 R-3,38-39.5$ & & & 270 & 3 & 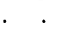 & 5 & 7 & 3 & 2 & 20 & 44 & 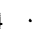 & & 3 & 4 & 4 & & . & & & 9 & & 5 & 9 & 1 & 46 & 11 & 4 & & 7 & \\
\hline & & & 11 & 3 & 2 & & 1 & 17 & 72 & & & 1 & & 4 & & & & 2 & & & & 9 & 3 & 1 & & 11 & 2 & & 4 & & \\
\hline $72 R-1,37.5-39$ & 769.375 & & 181 & 1 & 3 & 3 & 4 & 15 & 51 & & 8 & 3 & & & 3 & 6 & & . & & & & 2 & 17 & 1 & & 5 & & 12 & 6 & . & \\
\hline & 772.375 & & 179 & 2 & 5 & 1 & & 40 & 03 & & 3 & & & 4 & & & & & & & & 8 & 1 & & & 2 & 5 & 4 & 8 & & \\
\hline $72 \mathrm{R}-5,38-40$ & 775.38 & & 60 & & & & & 6 & & & & & & & & & & & & & & & 2 & & & 3 & & & & 3 & \\
\hline
\end{tabular}




\begin{tabular}{|c|c|c|c|c|c|c|c|c|c|c|c|c|c|c|c|c|c|c|c|c|c|c|c|c|c|c|c|c|c|c|c|c|}
\hline $\begin{array}{l}\text { Core, section, } \\
\text { interval }(\mathrm{cm})\end{array}$ & $\begin{array}{r}\text { Depth } \\
\text { (mbsf) } \\
\end{array}$ & 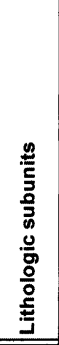 & 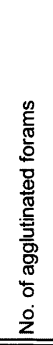 & 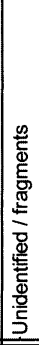 & 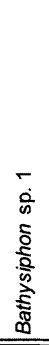 & 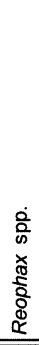 & 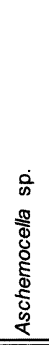 & 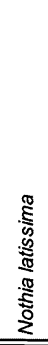 & 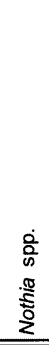 & 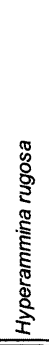 & 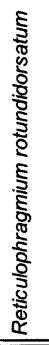 & 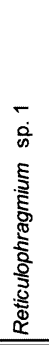 & 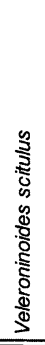 & 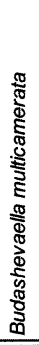 & 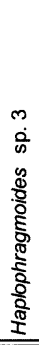 & 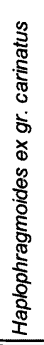 & 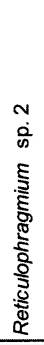 & 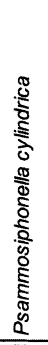 & 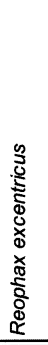 & 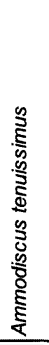 & 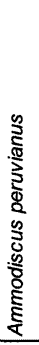 & 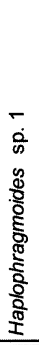 & 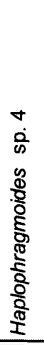 & 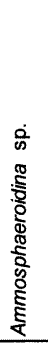 & 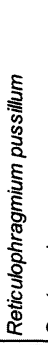 & 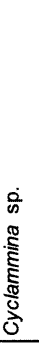 & 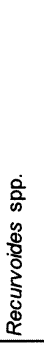 & 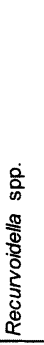 & 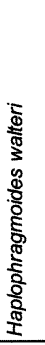 & 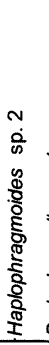 & 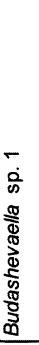 & 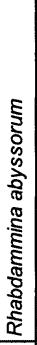 \\
\hline $73 R-1,38.5-40$ & 779.085 & \multirow{39}{*}{ IIIf } & 50 &. & . & . & $\cdot$ & 1 & 2 & 1 & . & 2 & 5 & $\overline{.}$ & . & . & 3 & $\overline{.}$ & . & $\bar{c}$ & . & . & 4 & $\overline{P \cdot}$ & 1 & . & 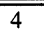 & . & 1 & 1 & . & $\overline{1 .}$ \\
\hline $73 R-3,38.5-40$ & 782.085 & & 137 & . & . & 2 & 4 & . & 4 & 3 & . & 2 & . & . & . & 2 & 5 & . & 4 & . & . & . & 14 & 6 & 3 & . & 4 & 4 & . & 12 & . & . \\
\hline 73R-5, 37.5-39 & 785.075 & & 214 & . & . & 1 & 6 & . & 12 & 10 & . & 6 & . & . & . & 4 & . & . & 2 & . & . & 6 & . & 4 & . & . & 4 & . & 6 & 24 & . & . \\
\hline $74 R-1,39.5-41$ & 788.695 & & 51 & . & . & 3 & $\cdot$ & . & $\cdot$ & 3 & . & $\cdot$ & . & . & 2 & 1 & 1 & . & . & . & . & . & 4 & $\cdot$ & . & . & 1 & 2 & . & $\cdot$ & . & . \\
\hline $74 R-3,40-41.5$ & 791.7 & & 183 & . & . & . & 10 & . & 4 & 2 & 1 & . & . & . & . & 2 & 2 & . & . & . & . & 3 & . & 16 & 10 & . & 3 & 4 & 10 & 12 & . & . \\
\hline $74 R-5,40-42$ & 794.7 & & 139 & . & 1 & 1 & $\cdot$ & . & . & 5 & . & 1 & . & . & . & 4 & . & . & . & . & . & 3 & . & 1 & 1 & . & . & . & 4 & 2 & . & 2 \\
\hline $75 R-1,40-41.5$ & 798.4 & & 157 & . & 6 & 2 & $\cdot$ & $\cdot$ & . & 3 & $\cdot$ & 5 & . & . & . & 10 & 9 & . & . & 2 & 1 & 3 & $\cdot$ & $\cdot$ & . & . & . & . & 12 & 5 & . & . \\
\hline $75 R-3,40-42$ & 801.4 & & 97 & $\cdot$ & . & 2 & 10 & · & 3 & 1 & $\cdot$ & 1 & . & . & . & 4 & $\cdot$ & . & . & . & . & . & . & 2 & . & . & . & 3 & 4 & 7 & . & . \\
\hline $75 R-5,40-41.5$ & 804.4 & & 99 & . & . & 1 & 2 & 1 & 5 & 1 & . & 2 & . & . & . & 6 & . & . & . & 1 & . & 6 & . & 2 & . & 1 & . & . & . & 4 & . & . \\
\hline $76 \mathrm{R}-1,40-41.5$ & 808 & & 52 & . & . & . & 4 & . & . & 5 & 1 & 3 & . & . & 1 & 1 & 2 & . & . & . & . & . & . & . & 5 & . & 1 & . & 6 & 2 & . & . \\
\hline $76 R-3,40-41.5$ & 811 & & 241 & . & . & 1 & 1 & 4 & 18 & 11 & . & . & . & . & . & 10 & . & . & 2 & 1 & . & 4 & 1 & 11 & 20 & . & . & . & 11 & . & . & . \\
\hline $76 R-5,40-41.5$ & 814 & & 143 & . & . & . & 2 & 1 & 12 & 24 & . & 5 & . & . & . & 30 & . & . & . & 2 & . & . & . & . & . & . & 1 & . & 15 & 14 & . & . \\
\hline $77 R-1,40-41.5$ & 817.7 & & 18 & . & . & . & . & . & 2 & 2 & . & . & 1 & . & . & 2 & 3 & . & . & . & . & . & . & . & . & . & 1 & . & . & 1 & . & . \\
\hline $77 R-3,40-41.5$ & 820.7 & & 146 & . & . & 3 & . & 2 & 15 & 16 & . & . & 1 & . & . & 6 & 3 & . & 6 & . & . & 2 & . & 5 & . & . & 5 & . & 8 & 9 & . & . \\
\hline $77 R-5,36-37.5$ & 823.66 & & 66 & . & . & . & . & 1 & 6 & 4 & . & 6 & . & . & . & 9 & 1 & . & 1 & 1 & . & . & . & 3 & . & . & . & . & 3 & . & . & . \\
\hline $78 R-1,40-42$ & 827.4 & & 74 & . & . & 1 & . & 3 & 2 & . & . & . & . & . & 1 & 6 & 5 & . & 1 & . & . & . & 4 & 2 & 1 & & 1 & . & 1 & 1 & . & . \\
\hline $78 R-2,40-42$ & 828.9 & & 143 & & 2 & 3 & 2 & 12 & 34 & 12 & . & $\cdot$ & 1 & . & . & 9 & 5 & . & 2 & 1 & . & . & 2 & 5 & 2 & . & . & . & 11 & 3 & . & . \\
\hline $78 R-3,37-39$ & 830.37 & & 156 & . & . & 2 & $\cdot$ & 4 & 10 & 10 & . & . & . & . & . & 13 & 5 & . & 2 & 4 & 1 & . & . & 5 & . & . & . & . & 7 & . & . & . \\
\hline $80 R-1,40-41.5$ & 846.6 & & 104 & . & . & . & . & 5 & 5 & 8 & . & . & . & . & . & 10 & . & . & 8 & . & . & . & . & 8 & . & & 20 & . & 2 & . & 2 & . \\
\hline $80 R-3,40-42$ & 849.6 & & 159 & . & 1 & 1 & . & 8 & 6 & 7 & . & . & . & 1 & . & 32 & 12 & . & 3 & 2 & . & . & . & . & 1 & . & 9 & . & 11 & 4 & . & . \\
\hline $80 R-5,38.5-40$ & 852.585 & & 110 & . & . & 2 & 2 & $\cdot$ & 11 & 6 & . & . & 2 & . & . & 10 & 1 & . & . & 1 & . & 2 & . & 2 & 1 & & 12 & 11 & . & 3 & . & . \\
\hline $81 R-1,40.5-42$ & 856.3 & & 116 & . & . & 7 & . & 1 & 7 & 2 & . & . & . & . & 2 & $\cdot$ & 7 & . & 1 & . & . & . & . & 3 & 3 & . & 9 & . & 7 & 4 & . & . \\
\hline $81 R-3,40-42$ & 859.3 & & 88 & . & . & . & . & . & 2 & . & . & . & 3 & . & . & 15 & 5 & 1 & $\cdot$ & 2 & . & . & . & 2 & 2 & 1 & 6 & . & 2 & 5 & 1 & . \\
\hline $81 R-4,39-40.5$ & 860.79 & & 215 & . & . & 2 & . & 3 & 1 & . & . & . & 9 & . & . & 34 & 4 & $\cdot$ & 16 & $\cdot$ & 3 & . & 1 & 14 & . & . & 15 & . & . & . & . & . \\
\hline $82 R-1,40-41.5$ & 865.9 & & 131 & . & 1 & 1 & . & . & 7 & 31 & . & 1 & 2 & . & . & 15 & . & . & 3 & 15 & 5 & . & . & 5 & . & 1 & 9 & . & . & 5 & . & . \\
\hline $82 R-2,40-42$ & 867.4 & & 183 & 9 & . & 1 & . & . & 7 & 1 & . & . & . & . & . & 10 & . & 1 & 9 & 2 & 1 & 8 & 5 & 10 & . & . & 14 & . & 3 & 8 & . & . \\
\hline $83 R-1,40.5-42$ & 875.5 & & 133 & 2 & . & 1 & . & 2 & 10 & . & . & 2 & 2 & . & . & 1 & . & . & 7 & . & . & . & . & 12 & . & . & 14 & . & 9 & . & 1 & . \\
\hline $83 R-3,41-42.5$ & 878.51 & & 81 & $\cdot$ & 2 & . & . & $\cdot$ & 7 & 3 & . & . & 14 & . & . & 22 & 2 & . & 1 & . & . & . & . & 2 & . & . & 6 & . & . & . & . & . \\
\hline $83 R-4,40-42$ & 880 & & 162 & 19 & . & . & . & 2 & 3 & 6 & . & 1 & $\cdot$ & . & . & 18 & . & 2 & 3 & . & . & . & $\cdot$ & 10 & 4 & . & 4 & . & . & 5 & . & . \\
\hline $84 R-1,40-42$ & 885.2 & & 161 & . & . & 2 & . & 1 & 3 & 3 & . & . & 13 & . & . & 10 & 2 & . & 6 & . & . & 1 & 3 & 2 & 2 & 2 & 13 & . & 3 & . & . & . \\
\hline $84 R-3,38-40$ & 888.18 & & 139 & . & 1 & 2 & . & $\cdot$ & 11 & 2 & . & $\cdot$ & . & . & . & 8 & . & 1 & 5 & . & . & . & . & 23 & 2 & . & 5 & . & 4 & 6 & . & . \\
\hline $84 R-5,30-32$ & 891.1 & & 136 & . & . & 1 & . & 3 & 9 & 5 & . & 4 & . & . & . & 9 & . & . & 6 & . & 2 & . & 3 & 11 & . & . & . & . & 1 & 3 & . & . \\
\hline $85 R-1,40-41.5$ & 894.8 & & 144 & . & . & 3 & . & . & 8 & 4 & . & . & . & . & . & 5 & . & $\cdot$ & 13 & 2 & 1 & . & . & 3 & . & & 7 & . & 2 & . & . & . \\
\hline $85 R-2,40-42$ & 896.3 & & 160 & . & . & 1 & . & $\cdot$ & 15 & 8 & . & . & . & . & . & 13 & . & . & 3 & . & . & 2 & . & . & . & & 4 & . & 5 & . & 9 & . \\
\hline $86 R-1,41-42.5$ & 904.51 & & 190 & . & 1 & . & 6 & 5 & 1 & 3 & . & . & . & 3 & 4 & 24 & . & . & 8 & 2 & 3 & . & . & 10 & 15 & & 12 & . & . & $\cdot$ & 13 & . \\
\hline $86 R-2,40-41.5$ & 906 & & 134 & 8 & . & . & . & . & . & 12 & . & . & . & 2 & . & 5 & 1 & . & 7 & . & 2 & . & . & 8 & 9 & & 8 & . & . & . & 8 & . \\
\hline $86 R-3,40-42$ & 907.5 & & 197 & 10 & . & 7 & 1 & 5 & 2 & 2 & . & . & 3 & . & . & 27 & . & $\cdot$ & 10 & 2 & 1 & . & . & 26 & 3 & . & 7 & . & . & . & 1 & . \\
\hline $87 R-1,40-42$ & 914.2 & & 98 & 10 & 1 & 2 & . & 4 & 2 & 1 & . & . & . & 2 & . & 4 & . & . & . & 6 & . & . & $\cdot$ & 3 & 7 & . & 16 & . & . & . & . & . \\
\hline $87 R-2,40-42$ & 915.7 & & 316 & 20 & 3 & . & . & 1 & 5 & 7 & . & . & . & 4 & . & 9 & . & . & 5 & 1 & 2 & . & . & 7 & 2 & & 7 & . & . & . & 3 & . \\
\hline $88 R-1,39.5-41$ & 923.795 & & 10 & 1 & . & . & . & . & . & . & . & . & . & . & . & . & . & . & 1 & . & . & 2 & . & 1 & . & . & 1 & . & . & . & . & . \\
\hline $88 R-2,40.5-42$ & 925.305 & & 34 & 2 & . & . & . & . & 1 & 2 & . & . & . & . & . & . & . & . & . & . & . & . & . & . & . & 1 & . & . & . & . & . & . \\
\hline $89 R-1,40-42$ & 933.5 & & 5 & . & . & . & . & . & . & . & . & . & . & . & . & . & 2 & . & . & . & . & 1 & . & . & . & . & 1 & . & . & . & . & . \\
\hline $89 R-2,40-41.5$ & 935 & & 8 & . & . & . & . & . & 2 & . & . & . & . & . & . & . & . & . & . & . & . & . & . & . & . & . & . & . & . & . & . & . \\
\hline $90 R-1,41-42.5$ & 943.21 & & 23 & 1 & . & . & . & . & 5 & 1 & . & . & . & . & . & . & . & . & . & . & . & 1 & . & . & . & . & . & . & . & . & . & . \\
\hline $90 \mathrm{R}-2,40-41.5$ & 944.7 & & 0 & . & . & . & . & . & . & . & . & . & . & . & . & . & . & . & . & . & . & . & . & . & . & . & . & . & . & . & . & . \\
\hline $91 \mathrm{R}-1,38.5-40.5$ & 952.785 & & 177 & 1 & 2 & 3 & . & . & 3 & 17 & . & . & . & . & . & 16 & . & . & 5 & 2 & . & 1 & . & 13 & . & & 19 & . & . & . & 1 & . \\
\hline $91 R-2,38-40$ & 954.28 & & 227 & 9 & 1 & 2 & 1 & . & 4 & 22 & . & . & . & . & . & 21 & . & . & 4 & 1 & . & . & . & 18 & . & . & 17 & . & . & . & 4 & 10 \\
\hline $92 R-1,38.5-40.5$ & 962.485 & & 176 & 5 & 6 & 1 & . & . & 5 & 9 & . & 4 & . & . & . & 12 & 1 & . & 8 & . & . & 4 & . & 14 & 3 & 1 & 8 & $\cdot$ & . & . & 2 & . \\
\hline $92 R-2,38-40$ & 963.98 & & 193 & . & 7 & 7 & 1 & . & 12 & . & . & . & . & . & . & 7 & . & . & . & . & 1 & 1 & . & $\cdot$ & 16 & . & . & 13 & . & . & . & . \\
\hline $93 R-1,40-41.5$ & 972.1 & & 223 & . & 6 & 1 & 4 & . & 24 & 18 & 3 & 1 & . & . & . & 14 & . & . & 3 & 1 & . & 2 & . & 9 & 1 & & 3 & . & . & . & . & . \\
\hline $93 R-2,36-37.5$ & 973.56 & IIIE & 276 & 6 & 6 & . & 2 & . & 6 & 15 & 3 & . & . & . & . & 23 & . & . & . & 1 & . & 4 & . & 8 & . & & 4 & . & . & . & . & 2 \\
\hline $94 R-1,40-41.5$ & 981.7 & & 86 & 5 & 2 & . & . & . & 1 & 4 & . & . & . & . & . & 8 & . & . & . & 1 & . & 2 & . & 5 & . & & 12 & . & . & . & . & . \\
\hline $94 R-2,40-41.5$ & 983.2 & & 162 & 6 & 1 & 7 & . & . & 7 & 10 & . & . & . & . & . & 9 & . & . & 5 & . & . & 1 & . & 9 & 1 & & 5 & . & . & . & 2 & . \\
\hline $94 R-3,35-36.5$ & 984.65 & & 220 & 10 & 2 & 6 & 4 & $\cdot$ & 12 & 9 & . & . & . & . & 9 & 14 & . & . & 9 & . & . & . & . & 4 & 2 & 1 & 8 & . & . & . & 9 & 2 \\
\hline $95 R-1,42-43.5$ & 991.12 & & 153 & 4 & . & 1 & 1 & 12 & 14 & 12 & 1 & . & . & . & 1 & 3 & . & . & 4 & 2 & . & 6 & . & 1 & . & . & 2 & . & . & . & 3 & . \\
\hline $95 R-2,22-23.5$ & 992.42 & & 112 & 13 & . & 1 & 1 & 4 & 6 & 3 & . & . & . & . & . & 6 & 5 & 8 & 1 & 1 & 2 & 6 & . & 3 & 1 & & 7 & . & . & . & . & . \\
\hline $96 R-1,40-41.5$ & 1000.8 & & 150 & 10 & . & . & . & 6 & 13 & 22 & . & . & 1 & . & 1 & 9 & . & . & 5 & 2 & 1 & 1 & . & 3 & 2 & & 23 & . & . & . & 1 & . \\
\hline $97 R-1,40-41.5$ & 1010.4 & & 97 & 6 & . & 2 & . & 22 & 22 & 5 & . & . & . & 1 & . & 6 & . & . & 3 & 1 & . & . & . & 2 & . & 1 & 7 & . & . & . & 3 & . \\
\hline $98 R-1,37-38.5$ & 1020.07 & & 184 & 7 & 5 & 2 & 10 & 8 & 17 & 15 & 1 & . & . & . & . & 11 & 3 & 15 & 11 & 2 & . & . & . & 3 & 5 & & 20 & . & . & . & 2 & . \\
\hline $98 \mathrm{R}-2,38.5-40$ & 1021.585 & & 61 & 4 & 1 & 7 & $\cdot$ & 1 & 4 & 3 & · & 2 & . & . & . & 6 & . & $\cdot$ & $\cdot$ & 1 & . & . & . & 1 & 2 & & 4 & . & . & 5 & 4 & . \\
\hline $99 R-1,37-38.5$ & 1029.57 & & 59 & 6 & 1 & 1 & . & 2 & 9 & 1 & . & . & . & . & . & 4 & . & . & . & . & 1 & . & . & . & . & & 11 & . & . & . & 3 & . \\
\hline $100 R-1,33-35$ & 1039.13 & & 78 & - & 1 & 8 & . & 5 & . & 12 & . & . & . & . & . & 1 & . & . & . & 1 & 1 & . & . & 1 & . & & 17 & . & . & . & 8 & . \\
\hline $100 R-2,41-42.5$ & 1040.71 & & 12 & . & . & . & . & . & . & . & . & . & . & . & . & & . & . & . & . & . & . & . & 1 & . & & & . & . & . & 4 & . \\
\hline
\end{tabular}


APPENDIX 1

ODP Hole 909 data.

\begin{tabular}{|c|c|c|c|c|c|c|c|c|c|c|c|c|c|c|c|c|c|c|c|c|c|c|c|c|c|c|c|c|c|}
\hline $\begin{array}{l}\text { Core, section, } \\
\text { interval }(\mathbf{c m})\end{array}$ & $\begin{array}{r}\text { Depth } \\
\text { (mbsf) }\end{array}$ & 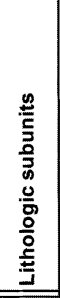 & 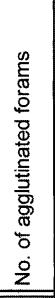 & 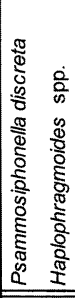 & 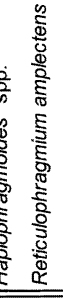 & 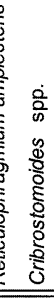 & 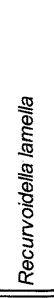 & 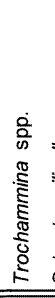 & 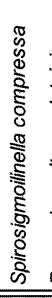 & 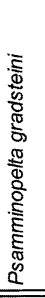 & 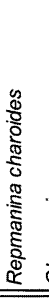 & 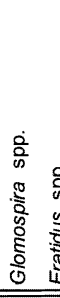 & 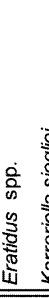 & 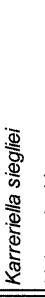 & 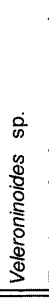 & 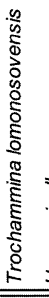 & 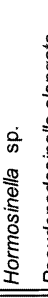 & 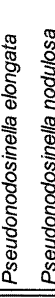 & 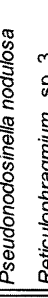 & 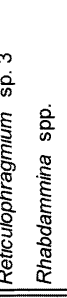 & 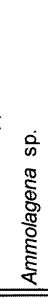 & 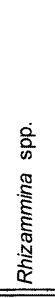 & 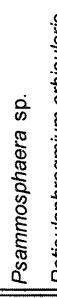 & 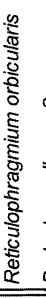 & 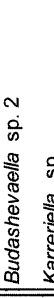 & is & 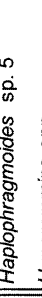 & & 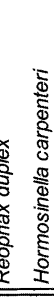 \\
\hline 151-909C- & & & & & & & & & & & & & & & & & & & & & & & & & & & & & \\
\hline $51 \mathrm{R}-1,37-39$ & 66.97 & & 0 & . & - & . & . & $\cdot$ & . & $\cdot$ & - & . & . & & - & . & $\cdot$ & & & & & & & & & & & & \\
\hline $51 R-3,38.5-40$ & 569.985 & & 0 & . & . & . & . & . & . & . & . & . & . & & & . & . & - & & & . & - & & . & - & & & & \\
\hline 51R-5, 35-37 & 572.95 & & 0 & . & . & . & & . & . & . & . & . & . & $\cdot$ & . & . & . & - & . & - & . & . & - & - & . & & & - & \\
\hline $52 R-1,38.5-40$ & 576.585 & & 0 & . & . & . & . & . & . & . & . & . & . & . & . & . & . & - & . & . & . & . & & . & . & & & & \\
\hline $52 R-3,38.5-40$ & 579.585 & & 0 & . & . & . & . & . & . & . & . & . & . & . & . & . & . & & . & . & . & $\cdot$ & . & $\cdot$ & - & & & - & \\
\hline $52 \mathrm{R}-4,35.5-37$ & 581.055 & & 0 & . & . & . & & . & . & . & & . & . & . & . & . & . & . & . & . & . & . & - & . & . & & . & - & \\
\hline $53 R-1,37.5-39.5$ & 586.175 & & 0 & . & . & . & . & . & . & . & . & . & . & . & - & . & . & & - & . & - & . & . & . & . & & & - & \\
\hline $53 R-3,38-40$ & 589.18 & & 1 & . & . & . & . & . & & . & . & . & . & . & . & . & . & - & - & . & . & . & . & . & . & & . & & \\
\hline $53 R-5,38.5-40$ & 592.185 & & 0 & . & . & . & . & . & . & . & . & . & . & . & . & . & . & & - & . & . & . & - & . & . & & & - & \\
\hline $54 R-1,37-38.5$ & 595.87 & & 0 & . & . & . & & . & . & . & . & . & . & . & . & . & - & . & . & . & . & . & . & . & . & & . & - & \\
\hline $54 R-2,38-40$ & 597.38 & & 0 & . & . & . & & . & . & . & . & . & . & . & . & . & . & . & . & . & . & . & - & . & . & & & . & \\
\hline $54 \mathrm{R}-3,37-39$ & 598.87 & & 3 & . & - & . & . & . & - & . & . & . & . & . & . & . & . & . & . & . & . & . & . & - & . & . & . & - & \\
\hline $55 R-1,41.5-43$ & 605.515 & & 96 & . & - & . & . & . & . & . & . & . & . & . & . & . & . & . & . & . & - & . & . & . & . & & & . & \\
\hline $55 \mathrm{R}-3,40-41.5$ & 608.5 & & 87 & . & . & . & . & . & . & . & . & . & . & . & . & . & . & . & . & . & . & . & . & - & - & . & . & - & \\
\hline $55 R-5,38-39.5$ & 611.48 & & 3 & . & . & . & . & . & . & . & . & . & . & . & . & . & . & . & . & . & . & . & . & . & . & & & . & \\
\hline $56 R-1,43-45$ & 615.23 & & 0 & & . & . & . & . & . & . & . & . & . & & . & . & . & . & . & . & . & . & . & . & . & . & . & · & \\
\hline 57R-1, 37.5-39 & 624.775 & & 4 & . & . & . & . & . & . & & . & . & . & . & . & . & . & . & . & . & . & . & . & - & - & . & & - & \\
\hline 57R-3, 39-40.5 & 627.79 & & 0 & & . & . & . & - & . & . & . & . & . & . & . & . & . & - & . & - & - & . & . & - & - & - & . & . & \\
\hline 57R-5, 37-38.5 & 630.77 & & 0 & . & . & . & . & . & . & . & . & . & . & . & & . & . & & . & . & . & . & . & . & . & . & & & \\
\hline $58 \mathrm{R}-1,38.5-40$ & 634.485 & & 0 & & . & . & & . & . & . & & . & . & . & & . & . & . & . & . & . & . & . & . & . & . & . & . & \\
\hline $58 \mathrm{R}-2,38.5-40$ & 635.985 & IIIf & 0 & & . & . & . & . & . & . & . & . & . & . & & . & . & . & . & . & . & . & . & . & - & . & . & & \\
\hline $59 R-1,38.5-40$ & 644.085 & & 0 & & . & . & & . & . & . & & . & . & . & & & . & . & . & . & . & . & . & . & . & . & & & \\
\hline $59 R-3,38.5-40$ & 647.085 & & 54 & & . & . & . & . & . & . & . & . & . & . & & . & . & . & . & . & . & . & . & - & . & & . & & \\
\hline $59 R-5,37.5-39$ & 650.075 & & 107 & 4 & 1 & 4 & 3 & 1 & . & & . & . & & . & & . & . & . & . & . & . & . & & . & . & . & . & . & \\
\hline $60 R-1,38.5-40$ & 653.785 & & 47 & & . & . & . & 1 & 1 & 1 & 1 & 1 & . & . & & . & . & . & . & . & . & . & . & . & . & - & . & & \\
\hline $60 R-3,37.5-39$ & 656.775 & & 127 & & . & . & & 8 & . & & & . & 9 & 1 & & & . & . & . & . & . & . & . & . & . & . & . & . & \\
\hline $60 R-5,39-40.5$ & 659.79 & & 109 & . & . & . & . & 1 & . & . & . & . & 2 & & 9 & 3 & 1 & . & . & . & . & . & . & . & . & & . & & \\
\hline $61 R-1,38.5-40$ & 663.385 & & 1 & & . & . & . & . & . & . & . & . & . & . & & . & . & . & . & . & . & . & . & . & . & . & . & & \\
\hline $61 R-3,36-38$ & 666.36 & & 169 & . & . & - 11 & . & 11 & . & . & . & . & . & 3 & 7 & & 1 & 1 & 1 & & . & . & . & . & . & & . & & \\
\hline $61 R-5,37.5-39$ & 669.375 & & 58 & 1 & . & . . & & 6 & 1 & 1 & . & . & . & . & 3 & . & . & . & 1 & 3 & . & . & . & - & . & & . & & \\
\hline $62 R-1,38-40$ & 673.08 & & 1 & & . & . & . & . & . & . & . & . & . & . & . & . & . & . & . & . & . & . & . & . & . & & . & & \\
\hline $62 R-3,38.5-40$ & 676.085 & & 0 & & . & . & . & . & . & . & . & . & . & . & . & . & . & . & . & & . & . & & . & . & & . & & \\
\hline $62 R-5,38-40$ & 679.08 & & 156 & & . & 1 & & 10 & 1 & . & 2 & 5 & 2 & 2 & 2 & & 4 & . & . & - 10 & 0 & . & . & - & . & & . & & \\
\hline $63 R-1,42-43.5$ & 682.72 & & 73 & & . & . & . & $\cdot$ & . & . & . & . & . & 1 & 4 & . & 3 & . & 1 & 2. & . & . & . & . & . & & . & & . \\
\hline $63 R-3,43-44.5$ & 685.73 & & 67 & & . & . & & 3 & . & & . & . & 1 & 3 & 1 & . & . & . & $\cdot$ & & . & . & . & . & . & & . & & \\
\hline $63 R-4,37.5-39$ & 687.175 & & 156 & & . & . & & 18 & 4 & . & . & . & . & . & . & . & . & . & - 1 & 129 & 1 & . & & r. & . & . & . & · & \\
\hline $64 R-1,37.5-39$ & 692.375 & & 127 & & . & . & & 32 & 2 & . & . & & 1 & 6 & & . & . & . & . & 1. & . & 6 & & - & . & . & . & & \\
\hline $64 R-3,40-41.5$ & 695.4 & & 125 & . & . & 1 & . & 7 & . & . & . & & . & . & 3 & . & . & . & . & - 18 & . & . & 1 & . & . & - & . & · & . \\
\hline $64 R-5,38.5-40$ & 698.385 & & 132 & & . & . & & 10 & . & . & . & . & . & . & . & . & 1 & 2 & . & . $\cdot$ & 1 & . & & . & - & & . & & \\
\hline $65 R-1,38.5-40$ & 701.985 & & 69 & . & . & . & & 4 & . & . & . & . & 2 & 1 & . & . & . & 1 & . & 1 & . & . & . & . & . & . & . & · & \\
\hline $65 R-3,38.5-40$ & 704.985 & & 114 & & . & . & & 11 & . & . & . & . & 2 & . & . & . & . & . & . & . & . & . & . & . & - & & . & & \\
\hline $65 R-5,38.5-40$ & 707.985 & & 77 & & . & . & & 11 & . & . & . & . & . & . & 1 & . & 1 & . & . & 2 & 1 & . & 1 & . & . & . & . & - & \\
\hline $66 \mathrm{R}-1,38.5-40$ & 711.485 & & 146 & & . & . & & 5 & . & . & . & . & 1 & . & 1 & . & . & . & . & 1 & 2 & . & & 3 & 1 & . & . & & \\
\hline $66 R-2,36.5-38$ & 712.965 & & 80 & 1 & . & . & . & 9 & 3 & . & . & . & . & . & . & . & 3 & . & . & 3 & 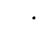 & . & & . & . & 1 & 5 & - & \\
\hline $67 R-1,40-41.5$ & 721.2 & & 166 & & . & . & & 19 & 5 & 1 & . & . & . & . & . & . & . & . & . & 3 & & . & . & . & 1 & & 8 & 4 & 2 \\
\hline $67 R-3,40-41.5$ & 724.2 & & 128 & . & 2 & . & . & 7 & & . & . & . & & 8 & . & . & . & 6 & . & · 43 & & 11 & . & . & 1 & & . & . & \\
\hline $67 R-5,39-40.5$ & 727.19 & & 155 & & & . & & 18 & & & . & & 1 & 4 & . & - & . & & . & . & & . & . & . & . & & 4 & 1 & \\
\hline $68 \mathrm{R}-1,40-41.5$ & 730.8 & & 137 & . & . & . & . & 3 & 1 & & . & - & & 1 & . & . & . & . & . & . & 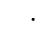 & 1 & . & . & . & & 3 & . & \\
\hline $68 R-3,40-41.5$ & 733.8 & & 82 & . & . & . & . & 6 & & . & . & . & & & . & . & . & & - & 21 & & & . & . & . & & . & 1 & \\
\hline $68 R-4,38.5-40$ & 735.285 & & 88 & & & 1 & & 12 & & 2 & 1 & & & & 1 & & 1 & & . & & & . & & . & & & & 1 & \\
\hline $69 R-1,38.5-40$ & 740.385 & & 62 & . & . & . & . & 2 & & & . & . & & . & . & . & . & . & . & 3 & & & 1 & . & - & & . & . & \\
\hline $69 R-3,40-41.5$ & 743.4 & & 75 & & . & . & . & 6 & . & . & . & . & & 5 & . & . & . & . & . & · 13 & & & 6 & . & . & & . & . & \\
\hline $69 R-5,38.5-40$ & 746.385 & & 111 & . & - & . & & 3 & & . & . & 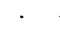 & & . & . & & . & 3 & & 44 & & 10 & & - & . & . & & 4 & \\
\hline $70 R-1,38.5-40$ & 749.985 & & 110 & . & . & . & . & 11 & & 5 & . & . & & . & . & & 4 & & . & 4 & 1 & 3 & & . & . & & & 2 & \\
\hline $70 R-3,37.5-39.5$ & 752.975 & & 91 & . & 1 & . & & 6 & 5 & & . & & 1 & 1 & . & . & . & & . & . & 1 & & 3 & . & . & & . & & \\
\hline $70 R-5,42.5-44$ & 756.025 & & 88 & . & . & . & . & . & 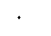 & . & . & . & & . & 5 & . & . & 2 & . & . 16 & & & 5 & & & & . & & \\
\hline $71 R-1,38.5-40$ & 759.685 & & 103 & . & 4 & . & 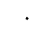 & 9 & & 1 & . & 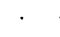 & & 4 & 2 & & 2 & 1 & . & - 12 & & & & & 3 & - & . & & \\
\hline $71 \mathrm{R}-3,38-39.5$ & 762.68 & & 270 & & - 12 & 21 & & 16 & & . & . & & 1 & . & 2 & - & . & & . & - 22 & 22 & - & 6 & & 2 & & & 3 & \\
\hline 71R-5, 38-40 & 765.68 & & 119 & . & 2 & & & 8 & 3 & 3 & . & 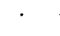 & & 1 & 2 & & 3 & & - & - 10 & 1 & 8 & 3 & - & & & & 2 & \\
\hline $72 \mathrm{R}-1,37.5-39$ & 769.375 & & 181 & . & 3 & 2 & & 10 & & 3 & . & & 1 & 6 & . & . & . & . & . & - 35 & 51 & 17 & . & . & . & & 1 & 1 & \\
\hline $72 \mathrm{R}-3,37.5-39$ & 772.375 & & 179 & 1 & 1 & . & & & & & & & & 3 & 3 & & 3 & 1 & & 44 & & 14 & 8 & & . & & & & \\
\hline
\end{tabular}


APPENDIX 1

ODP Hole 909 data.

\begin{tabular}{|c|c|c|c|c|c|c|c|c|c|c|c|c|c|c|c|c|c|c|c|c|c|c|c|c|c|c|c|c|}
\hline $\begin{array}{l}\text { Core, section, } \\
\text { interval (cm) }\end{array}$ & $\begin{array}{r}\text { Depth } \\
\text { (mbsf) }\end{array}$ & 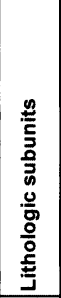 & 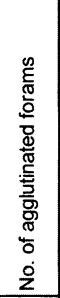 & 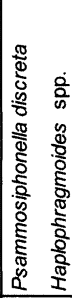 & 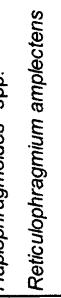 & 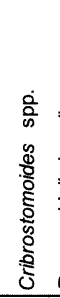 & 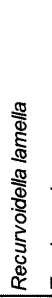 & 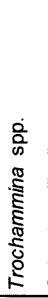 & 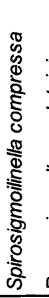 & 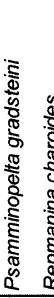 & 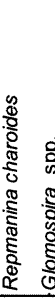 & 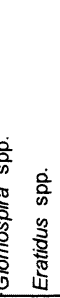 & 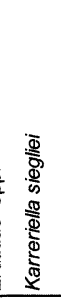 & 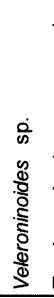 & 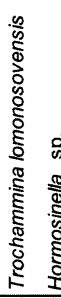 & 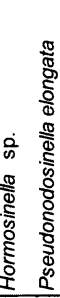 & 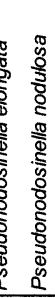 & 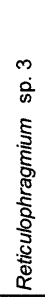 & 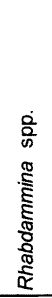 & 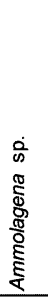 & & & 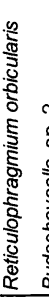 & 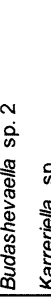 & 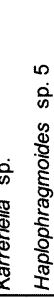 & 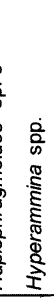 & 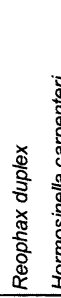 & 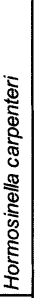 \\
\hline $73 R-1,38.5-40$ & 779.085 & & 50 & $\overline{c .}$ & . & & $\bar{c}$ & 8 & . & & . & 7. & 5 & 3 & . & 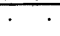 & & & 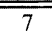 & & & & 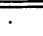 & ב. & ב. & 2 & 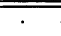 & \\
\hline $73 R-3,38.5-40$ & 782.085 & & 137 & . & . & . & & 16 & 2 & & . & . & . & 3 & . & . . & & & 18 & & 3 & 6 & & . & . & 11 & 1 & \\
\hline $73 R-5,37.5-39$ & 785.075 & IIIf & 214 & . & 1 & . & & 6 & & & & . & 4 & & . & . 10 & 0 & & 19 & . & & 10 & & . & . & 10 & . & \\
\hline $74 R-1,39.5-41$ & 788.695 & & 51 & . & 7 & . & & 6 & . & & & . & . & 6 & . & . . & & & 1 & . & & & . & . & . & 9 & . & \\
\hline $74 R-3,40-41.5$ & 791.7 & & 183 & . & 7 & . & & 12 & . & . & . & . & 2 & 9 & . & · 4 & 4 & & 33 & 2 & 19 & 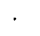 & & . & & 7 & . & \\
\hline $74 R-5,40-42$ & 794.7 & & 139 & 4 & +2 & . & & 24 & . & . & & . & . & & . & . & & & 63 & 1 & 12 & 6 & . & . & . & . & . & \\
\hline $75 R-1,40-41.5$ & 798.4 & & 157 & . & . & . & & 20 & . & . & . & . & 2 & . & . & 4 & 4 & & 30 & . & 24 & 2 & 1 & . & & 5 & . & \\
\hline $75 R-3,40-42$ & 801.4 & & 97 & . & . & . & & 17 & . & . & . & . & . & . & . & . & . & 4 & 17 & & 14 & & & . & 2 & 1 & . & \\
\hline $75 R-5,40-41.5$ & 804.4 & & 99 & & . & . & . & & . & . & . & & 8 & . & . & 3 & 3 & 2 & 39 & 1 & 7 & & & . & & 7 & . & \\
\hline $76 \mathrm{R}-1,40-41.5$ & 808 & & 52 & . & . & . & & 2 & . & . & 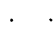 & $\cdot$ & 3 & . & . & 2 & 2 & . & 3 & & 2 & 2 & & . & . & 1 & . & \\
\hline $76 \mathrm{R}-3,40-41.5$ & 811 & & 241 & . & . & . & . & & 2 & 4 & . & 1 & 4 & 1 & . & . & & 1 & 1 & & \#\# & 4 & & . & & 17 & . & \\
\hline $76 R-5,40-41.5$ & 814 & & 143 & & . & . & . & & & . & . & & . & & . & 1 & 1 & 3 & 5 & & 5 & 7 & & . & . & 12 & . & \\
\hline $77 \mathrm{R}-1,40-41.5$ & 817.7 & & 18 & & 6 & . & . & & & . & . & & . & . & . & . & . & . & & . & . & 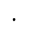 & & . & . & . & . & \\
\hline $77 \mathrm{R}-3,40-41.5$ & 820.7 & & 146 & & . & . & & . & 1 & . & s. & 1 & . & . & . & 1 & 1 & & 40 & . & 4 & 2 & & . & & . & . & \\
\hline $77 R-5,36-37.5$ & 823.66 & & 66 & & . & . & & 4 & . & . & . & & . & . & . & . & . & & 12 & . & 1 & 10 & & . & . & . & . & \\
\hline $78 R-1,40-42$ & 827.4 & & 74 & & 1 & . & & 4 & 2 & 5 & & & 4 & . & & 2 & 2 & 1 & 3 & . & 9 & 3 & & . & & 4 & . & \\
\hline $78 R-2,40-42$ & 828.9 & & 143 & & 1 & . & & 4 & & . & . & 2 & 2 & . & . & 3 & 3 & & 13 & & 5 & 2 & & . & & 2 & . & \\
\hline 78R-3, 37-39 & 830.37 & & 156 & & . & . & . & 7 & & . & . & & & . & . & 2 & 2 & 8 & & & 26 & 8 & & . & & 26 & 1 & \\
\hline $80 \mathrm{R}-1,40-41.5$ & 846.6 & & 104 & & . & . & . & & . & . & . & 1 & 1 & . & . & . & . & & 12 & . & 4 & 10 & & . & & 5 & . & \\
\hline $80 R-3,40-42$ & 849.6 & & 159 & & 12 & 1 & . & . & . & . & . & & 10 & 1 & . & . & . & . & & & 19 & 5 & & . & & 4 & . & \\
\hline $80 R-5,38.5-40$ & 852.585 & & 110 & . & . & . & . & . & . & . & . & 1 & . & . & . & 7 & 7 & 1 & 10 & . & 8 & 1 & & . & & 14 & . & \\
\hline $81 \mathrm{R}-1,40.5-42$ & 856.3 & & 116 & & . & . & & 2 & . & . & 2 & & 11 & & . & . & . & & 10 & 1 & 18 & 4 & & & & 6 & . & \\
\hline $81 R-3,40-42$ & 859.3 & & 88 & & . & . & 3 & 2 & . & . & . & & 3 & 14 & . & . & . & 1 & 4 & & 1 & 6 & & . & & 1 & . & \\
\hline $81 R-4,39-40.5$ & 860.79 & & 215 & & 1 & 11 & & 15 & . & . & . & 11 & 18 & 5 & . & . & . & 1 & 9 & & 10 & & & & & 11 & . & \\
\hline $82 R-1,40-41.5$ & 865.9 & & 131 & & . & . & & 5 & & . & . & & . & . & . & . & . & & 6 & . & 6 & 1 & & . & 1 & 3 & . & \\
\hline $82 R-2,40-42$ & 867.4 & & 183 & & 2 & . & & 21 & 1 & . & . & 3 & 10 & 4 & . & 3 & 3 & . & 14 & & 12 & 10 & & & & 3 & . & \\
\hline $83 R-1,40.5-42$ & 875.5 & & 133 & . & . & . & & 7 & . & . & . & 1 & . & . & . & . & . & . & 14 & . & 31 & 7 & & . & . & . & . & \\
\hline $83 R-3,41-42.5$ & 878.51 & & 81 & & . & . & & 5 & . & . & . & . & . & . & . & . & . & . & & & 8 & & & & & 2 & . & \\
\hline $83 R-4,40-42$ & 880 & & 162 & . & 4 & . & . & 11 & . & . & . & . & 6 & . & . & 2 & 2 & 1 & 21 & & & 14 & & . & & 6 & . & \\
\hline $84 \mathrm{R}-1,40-42$ & 885.2 & & 161 & & 1 & . & & 12 & . & 9 & . & 1 & 16 & . & . & 1 & 1 & & 26 & . & 5 & 8 & & . & & 5 & . & \\
\hline $84 \mathrm{R}-3,38-40$ & 888.18 & & 139 & . & 4 & 13 & . & 21 & . & . & . & . & . & . & . & 1 & 1 & . & 2 & . & & . & & . & . & . & . & \\
\hline $84 R-5,30-32$ & 891.1 & & 136 & . & . & 2 & 5 & 5 & . & . & . & . & . & & . & . & & & 32 & & 11 & 8 & & & & 2 & . & \\
\hline $85 R-1,40-41.5$ & 894.8 & & 144 & . & 4 & 8 & & 14 & . & . & . & . & 10 & & . & . & & . & 27 & & & 9 & & & & 6 & . & \\
\hline $85 R-2,40-42$ & 896.3 & & 160 & . & 1 & 5 & & 17 & . & . & . & . & 9 & & . & 3 & 3 & . & 13 & & 31 & & & & & 2 & . & \\
\hline $86 R-1,41-42.5$ & 904.51 & & 190 & . & 1 & 19 & & 9 & . & . & . & 2 & 3 & & . & 6 & 6 & . & 23 & & & 5 & & & & 2 & . & \\
\hline $86 \mathrm{R}-2,40-41.5$ & 906 & & 134 & . & 1 & 12 & & 8 & . & . & . & . & . & . & . & 5 & 5 & . & 22 & 2 & 2 & 7 & & & . & & . & \\
\hline $86 R-3,40-42$ & 907.5 & & 197 & . & : & 20 & & 14 & . & . & . & . & 8 & . & . & 1 & . & . & 32 & & & 5 & & . & & 2 & & \\
\hline $87 R-1,40-42$ & 914.2 & & 98 & . & 3 & 2 & & 10 & . & . & . & . & 2 & & . & 4 & 4 & . & 5 & & . & 6 & & & & 2 & & \\
\hline $87 R-2,40-42$ & 915.7 & & 316 & . & 20 & 3 & & 9 & . & . & . & 2 & 5 & 2 & . & 1 & $i$ & 1 & \# & & 33 & 2 & & & & 29 & & \\
\hline $88 R-1,39.5-41$ & 923.795 & & 10 & . & 1 & & . & . & . & . & . & . & . & . & . & & . & . & . & & . & 1 & & . & & 1 & . & \\
\hline $88 R-2,40.5-42$ & 925.305 & & 34 & . & . & . & . & 1 & . & . & . & . & . & . & . & 2 & . & . & 20 & & . & 1 & & & & 4 & . & \\
\hline $89 \mathrm{R}-1,40-42$ & 933.5 & & 5 & . & . & . & . & . & . & . & . & . & . & . & . & . & . & . & & . & . & & & & & & . & \\
\hline $89 R-2,40-41.5$ & 935 & & 8 & . & . & . & . & . & . & . & . & . & . & . & . & . & . & . & & . & . & . & & . & & . & . & \\
\hline $90 R-1,41-42.5$ & 943.21 & & 23 & . & . & . & . & . & . & . & . & . & . & . & . & . & . & 1 & 13 & . & . & 1 & & . & & & . & \\
\hline $90 \mathrm{R}-2,40-41.5$ & 944.7 & & 0 & . & . & . & . & . & . & . & . & . & . & . & . & . & . & . & & . & . & & & . & . & . & . & \\
\hline $91 \mathrm{R}-1,38.5-40.5$ & 952.785 & & 177 & . & 9 & 8 & . & 9 & & . & . & 6 & . & . & . & 3 & . & . & 28 & . & . & . & & . & & 14 & . & \\
\hline $91 \mathrm{R}-2,38-40$ & 954.28 & & 227 & . & 7 & 10 & . & 7 & & . & . & 2 & . & . & . & 3 & . & . & 20 & & 21 & 1 & & . & & 4 & 1 & \\
\hline $92 \mathrm{R}-1,38.5-40.5$ & 962.485 & & 176 & . & 11 & 3 & . & 2 & . & . & . & 3 & 4 & . & . & 2 & 2 & . & 6 & & 22 & & & 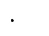 & & 15 & & \\
\hline $92 \mathrm{R}-2,38-40$ & 963.98 & & 193 & . & 5. & 5 & 7 & . & 9 & . & 2 & . & . & . & . & 3 & 5 & . & 27 & . & 5 & 32 & & . & & & 10 & \\
\hline $93 R-1,40-41.5$ & 972.1 & & 223 & . & 2 & 5 & . & . & & 1 & . & . & . & . & . & . & . & 1 & 12 & 1 & 81 & & & . & & 9 & & \\
\hline $93 R-2,36-37.5$ & 973.56 & IIIE & 276 & . & 4 & 1 & . & 2 & & 2 & . & . & 2 & . & . & 1 & 1 & & 4 & 2 & \#\# & & & & & 10 & . & \\
\hline $94 \mathrm{R}-1,40-41.5$ & 981.7 & & 86 & . & 4 & 2 & . & 3 & . & 5 & . & . & 1 & . & . & . & . & . & 6 & . & 5 & 6 & & . & & 7 & . & \\
\hline $94 \mathrm{R}-2,40-41.5$ & 983.2 & & 162 & . & 1 & 5 & . & 4 & . & 17 & . & 1 & 2 & . & . & 4 & 3 & & 14 & & 15 & 8 & & . & & 2 & . & \\
\hline $94 R-3,35-36.5$ & 984.65 & & 220 & & 3 & 3 & . & 17 & 3 & 2 & 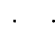 & & 13 & . & . & . & 4 & . & 34 & & 1 & 2 & & & 1 & 9 & 1 & \\
\hline $95 \mathrm{R}-1,42-43.5$ & 991.12 & & 153 & & 9 & 2 & . & . & . & 6 & . & 4 & 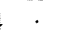 & . & & 1 & . & . & 16 & & 22 & & & & & 10 & & \\
\hline $95 \mathrm{R}-2,22-23.5$ & 992.42 & & 112 & . & 3 & 8 & & 7 & . & . & . & 1 & . & . & . & . & . & . & 1 & . & & 4 & & & & 13 & & \\
\hline $96 \mathrm{R}-1,40-41.5$ & 1000.8 & & 150 & . & 2 & 1 & . & 4 & . & . & . & 2 & . & . & . & . & 4 & . & 11 & . & . & 3 & & & & 10 & 1 & \\
\hline $97 \mathrm{R}-1,40-41.5$ & 1010.4 & & 97 & . & 1 & . & . & . & . & 2 & . & . & . & . & & . & . & . & . & . & 1 & . & . & . & & 9 & . & \\
\hline $98 R-1,37-38.5$ & 1020.07 & & 184 & . & 3 & 1 & & 11 & . & 1 & & 3 & 2 & & & 3 & . & . & 1 & . & 2 & . & & & & 3 & . & \\
\hline $98 \mathrm{R}-2,38.5-40$ & 1021.585 & & 61 & & 1 & 2 & & 2 & . & 5 & & 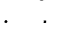 & . & & & . & 1 & . & . & . & & 2 & & & & 3 & . & \\
\hline $99 R-1,37-38.5$ & 1029.57 & & 59 & & 1 & . & & 3 & & . & . & . & 2 & & & . & . & . & 2 & . & . & 3 & . & & & 3 & . & \\
\hline $100 \mathrm{R}-1,33-35$ & 1039.13 & & 78 & . & . & . & . & & . & . & & 1 & . & . & & . & 1 & . & . & . & 5 & & & & & 8 & . & \\
\hline $100 \mathrm{R}-2,41-42.5$ & 1040.71 & & 12 & & . . & . & & & . & 3 & & & 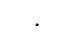 & . & . . &. . & . & & & . & & & & . & & & . & \\
\hline
\end{tabular}


APPENDIX 1

ODP Hole 909 data.

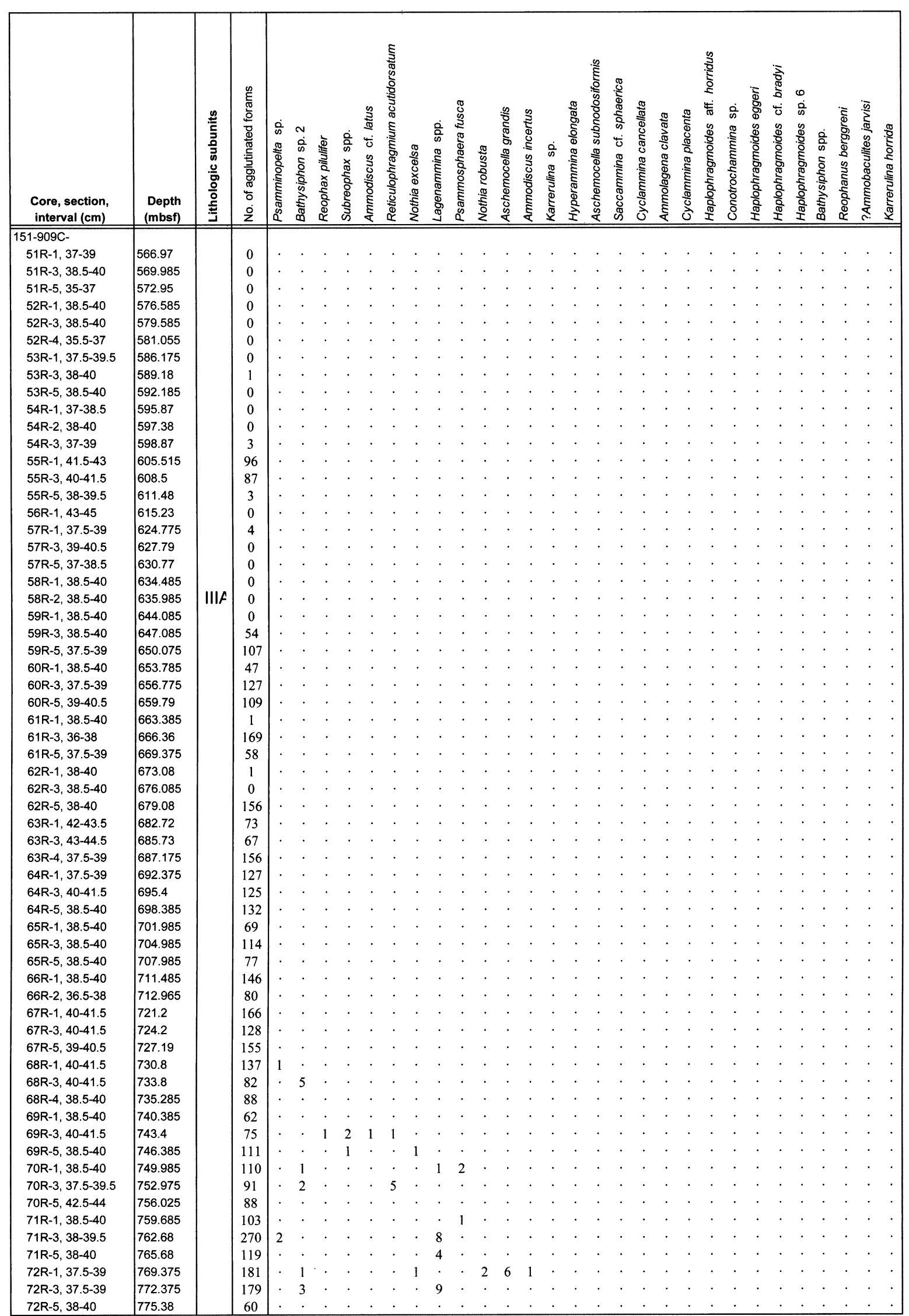




\begin{tabular}{|c|c|c|c|c|c|c|c|c|c|c|c|c|c|c|c|c|c|c|c|c|c|c|c|c|c|c|c|c|c|c|c|}
\hline $\begin{array}{c}\text { Core, section, } \\
\text { interval }(\mathbf{c m})\end{array}$ & $\begin{array}{r}\text { Depth } \\
\text { (mbsf) }\end{array}$ & 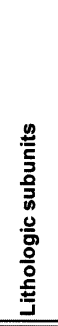 & 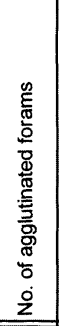 & 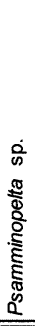 & 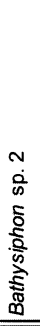 & 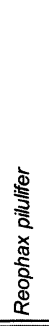 & 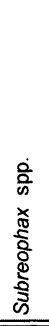 & 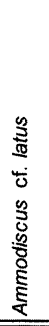 & 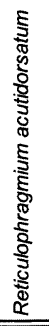 & 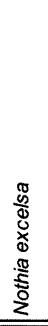 & 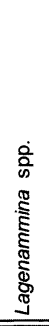 & 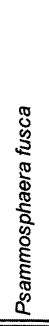 & 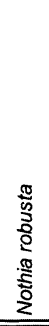 & 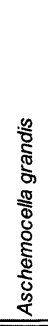 & 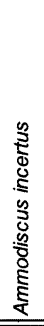 & 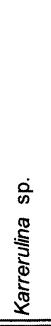 & 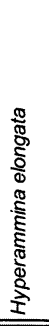 & 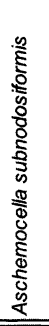 & 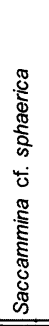 & 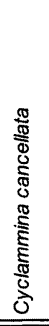 & 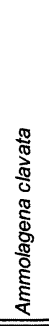 & 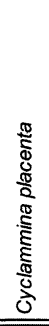 & 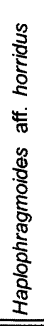 & 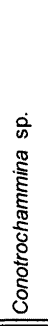 & 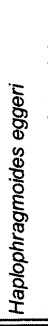 & 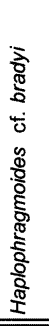 & 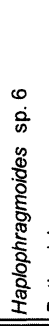 & 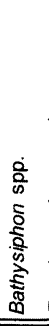 & 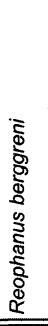 & 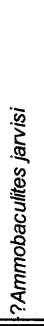 & 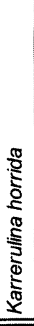 \\
\hline $73 R-1,38.5-40$ & 79.085 & & 50 & . & . & . & $\cdot$ & $\cdot$ & $\cdot$ & $\cdot$ & $\cdot$ & $\cdot$ & $\cdot$ & $\cdot$ & . & $\cdot$ & $\cdot$ & $\cdot$ &. & $\cdot$ &. &. & $\cdot$ & $\cdot$ & $\cdot$ & $\cdot$ & $\cdot$ & 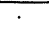 & $\cdot$ & . & \\
\hline $73 R-3,38.5-40$ & 782.085 & & 137 & . & . & . & . & . & . & . & 4 & 2 & . & . & . & 1 & 1 & $\cdot$ & . & . & . & . & . & . & . & . & . & . & . & . & \\
\hline $73 R-5,37.5-39$ & 785.075 & IIIf & 214 & . & . & 5 & . & . & . & . & . & . & . & . & . & $\cdot$ & 10 & 53 & . & . & . & . & . & . & . & . & . & . & . & . & . \\
\hline $74 \mathrm{R}-1,39.5-41$ & 788.695 & & 51 & $\cdot$ & . & 2 & . & . & 2 & . & 1 & . & . & . & . & $\cdot$ & $\cdot$ & $\cdot$ & . & . & . & . & . & . & . & . & · & . & . & . & . \\
\hline $74 R-3,40-41.5$ & 791.7 & & 183 & . & . & 1 & 6 & . & 1 & . & . & . & . & . & . & . & 1 & . & . & . & . & . & . & . & . & . & . & . & . & . & . \\
\hline $74 \mathrm{R}-5,40-42$ & 794.7 & & 139 & . & . & 1 & . & . & . & . & 1 & . & . & . & . & . & . & . & . & . & . & . & . & . & . & . & . & . & . & . & . \\
\hline $75 R-1,40-41.5$ & 798.4 & & 157 & . & . & 1 & . & $\cdot$ & 3 & . & 3 & . & . & . & . & . & 4 & . & . & . & . & . & . & . & . & . & $\cdot$ & . & . & $\cdot$ & \\
\hline $75 R-3,40-42$ & 801.4 & & 97 & . & 4 & . & $\cdot$ & $\cdot$ & 1 & . & . & . & . & . & . & $\cdot$ & . & . & . & . & . & . & . & . & . & . & · & . & . & . & \\
\hline $75 R-5,40-41.5$ & 804.4 & & 99 & . & . & . & . & . & . & . & . & . & . & . & . & . & . & . & . & . & . & . & . & . & . & . & . & . & . & . & \\
\hline $76 R-1,40-41.5$ & 808 & & 52 & . & $\cdot$ & 1 & . & . & . & . & 5 & . & . & . & . & . & . & . & . & . & . & . & . & . & . & . & . & . & . & . & \\
\hline $76 R-3,40-41.5$ & 811 & & 241 & . & . & 1 & . & . & 1 & . & 3 & . & . & . & . & . & 2 & 2 & . & . & . & . & . & . & . & . & . & . & . & . & \\
\hline $76 R-5,40-41.5$ & 814 & & 143 & . & . & . & . & . & . & . & 4 & . & . & . & . & . & . & . & . & . & . & . & . & . & . & · & $\cdot$ & . & . & · & \\
\hline $77 R-1,40-41.5$ & 817.7 & & 18 & . & . & $\cdot$ & . & . & . & . & . & $\cdot$ & . & . & . & . & $\cdot$ & $\cdot$ & $\cdot$ & . & $\cdot$ & . & . & . & . & . & $\cdot$ & . & . & . & \\
\hline 77R-3, 40-41.5 & 820.7 & & 146 & . & . & 3 & . & . & . & 1 & 2 & . & . & . & . & . & $\cdot$ & 10 & $\cdot$ & . & . & . & . & . & . & . & $\cdot$ & . & . & . & \\
\hline $77 R-5,36-37.5$ & 823.66 & & 66 & . & . & 1 & . & . & . & . & 3 & . & . & . & . & . & $\cdot$ & $\cdot$ & . & . & . & . & . & . & . & . & . & . & . & . & tra \\
\hline $78 R-1,40-42$ & 827.4 & & 74 & . & . & 2 & . & 1 & $\cdot$ & . & 4 & . & . & . & . & . & . & . & . & . & . & . & . & . & . & . & . & . & . & . & . \\
\hline $78 R-2,40-42$ & 828.9 & & 143 & . & . & . & . & . & $\cdot$ & 1 & 2 & . & . & . & . & . & . & . & . & . & . & . & . & . & . & . & $\cdot$ & . & . & . & . \\
\hline $78 R-3,37-39$ & 830.37 & & 156 & . & . & 4 & . & · & 3 & $\cdot$ & . & . & . & . & . & . & . & . & . & . & . & . & . & . & . & . & . & . & . & . & \\
\hline $80 R-1,40-41.5$ & 846.6 & & 104 & . & . & . & . & . & 1 & . & 1 & . & . & . & . & . & . & . & 1 & . & . & . & . & . & . & · & . & . & . & . & \\
\hline $80 R-3,40-42$ & 849.6 & & 159 & . & . & 8 & . & . & . & . & . & . & . & . & . & . & . & . & . & 1 & . & . & . & . & . & . & . & . & . & . & \\
\hline $80 R-5,38.5-40$ & 852.585 & & 110 & . & . & 1 & . & . & . & . & . & . & . & . & . & . & $\cdot$ & . & $\cdot$ & . & $\cdot$ & . & . & . & . & . & . & . & . & . & \\
\hline $81 R-1,40.5-42$ & 856.3 & & 116 & . & $\cdot$ & 5 & $\cdot$ & $\cdot$ & . & $\cdot$ & 3 & $\cdot$ & $\cdot$ & . & . & $\cdot$ & $\cdot$ & . & . & . & 1 & . & . & . & . & . & . & . & . & . & \\
\hline $81 R-3,40-42$ & 859.3 & & 88 & . & . & 2 & . & . & $\cdot$ & $\cdot$ & 3 & . & . & . & . & . & . & . & . & 1 & $\cdot$ & . & . & . & . & . & $\cdot$ & $\cdot$ & . & . & 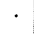 \\
\hline $81 R-4,39-40.5$ & 860.79 & & 215 & 7 & . & 9 & . & . & 1 & $\cdot$ & 10 & . & . & . & . & . & 4 & . & . & . & . & . & . & . & . & . & . & . & . & . & \\
\hline $82 R-1,40-41.5$ & 865.9 & & 131 & . & . & 2 & . & . & . & . & 4 & . & 2 & . & . & . & . & . & . & . & . & . & . & . & . & . & . & . & . & . & \\
\hline $82 R-2,40-42$ & 867.4 & & 183 & . & . & 2 & . & . & . & . & 9 & . & . & · & . & . & . & . & . & . & . & · & . & . & . & . & . & . & . & . & \\
\hline $83 R-1,40.5-42$ & 875.5 & & 133 & . & . & 3 & $\cdot$ & . & . & . & 5 & . & . & . & . & . & 1 & . & . & . & · & . & . & . & . & . & . & . & . & . & · \\
\hline $83 R-3,41-42.5$ & 878.51 & & 81 & . & . & $\cdot$ & . & . & . & . & 7 & . & . & . & . & . & . & . & . & . & . & . & . & . & . & . & . & . & . & . & . \\
\hline $83 R-4,40-42$ & 880 & & 162 & . & . & 10 & . & . & 1 & . & 3 & . & . & . & . & . & . & 4 & . & . & . & . & . & . & . & . & . & . & . & . & . \\
\hline $84 R-1,40-42$ & 885.2 & & 161 & . & . & 5 & . & $\cdot$ & . & $\cdot$ & 5 & . & . & . & . & . & . & . & . & . & . & 1 & . & . & . & . & . & . & . & . & . \\
\hline $84 R-3,38-40$ & 888.18 & & 139 & . & . & 10 & . & . & . & $\cdot$ & 10 & . & . & . & . & . & . & 6 & . & 2 & . & . & . & . & . & . & . & . & . & . & . \\
\hline $84 R-5,30-32$ & 891.1 & & 136 & . & $\cdot$ & 6 & $\cdot$ & . & $\cdot$ & $\cdot$ & 1 & . & . & . & . & . & 1 & 6 & . & · & $\cdot$ & . & $\cdot$ & . & $\cdot$ & . & . & . & . & . & . \\
\hline $85 R-1,40-41.5$ & 894.8 & & 144 & . & $\cdot$ & 10 & . & . & 5 & 1 & 1 & . & . & 1 & . & . & . & . & . & . & . & . & . & . & . & . & . & . & . & . & . \\
\hline $85 R-2,40-42$ & 896.3 & & 160 & . & $\cdot$ & 7 & 1 & . & . & . & 8 & . & 2 & . & . & . & . & . & . & . & . & . & 1 & . & . & . & . & . & . & . & \\
\hline $86 R-1,41-42.5$ & 904.51 & & 190 & . & . & 5 & . & . & . & . & 4 & . & 1 & . & . & . & . & . & . & . & . & . & $\cdot$ & . & . & . & . & . & . & . & . \\
\hline $86 R-2,40-41.5$ & 906 & & 134 & . & . & . & . & . & 3 & . & . & . & . & . & . & . & . & . & . & . & . & 2 & . & . & . & . & . & . & . & . & . \\
\hline $86 R-3,40-42$ & 907.5 & & 197 & . & . & 2 & . & . & . & . & 2 & . & . & . & . & . & . & 2 & . & . & . & 1 & 1 & . & . & . & . & . & . & . & \\
\hline $87 R-1,40-42$ & 914.2 & & 98 & . & 1 & . & . & . & 1 & . & . & . & . & . & . & . & . & . & . & . & . & 1 & 1 & 2 & . & . & . & . & . & . & \\
\hline $87 R-2,40-42$ & 915.7 & & 316 & . & 2 & 16 & . & 1 & . & 1 & 6 & . & . & . & . & . & . & 1 & . & . & . & . & 1 & . & . & . & . & . & . & . & . \\
\hline $88 R-1,39.5-41$ & 923.795 & & 10 & 1 & . & $\cdot$ & . & . & $\cdot$ & $\cdot$ & . & . & . & $\cdot$ & . & $\cdot$ & $\cdot$ & . & · & . & · & . & . & . & . & . & . & . & . & . & . \\
\hline $88 R-2,40.5-42$ & 925.305 & & 34 & $\cdot$ & $\cdot$ & . & . & . & $\cdot$ & . & . & . & . & . & . & . & . & . & . & . & . & . & $\cdot$ & . & . & . & . & . & · & · & . \\
\hline $89 R-1,40-42$ & 933.5 & & 5 & . & . & . & . & . & . & . & . & . & 1 & . & . & . & . & . & . & . & · & . & . & . & . & . & . & . & . & . & . \\
\hline $89 R-2,40-41.5$ & 935 & & 8 & . & . & . & . & . & . & . & . & . & . & . & . & . & . & 6 & . & . & . & . & . & . & . & . & . & . & . & . & . \\
\hline $90 R-1,41-42.5$ & 943.21 & & 23 & . & . & . & . & . & . & . & . & . & . & . & . & . & . & . & . & . & . & . & . & . & . & . & . & . & . & . & . \\
\hline $90 R-2,40-41.5$ & 944.7 & & 0 & . & . & . & . & . & . & . & . & . & . & . & . & . & . & . & . & . & . & . & . & . & . & . & . & . & . & . & . \\
\hline $91 R-1,38.5-40.5$ & 952.785 & & 177 & . & . & 1 & . & . & . & 4 & 8 & . & 1 & . & . & . & . & 1 & $\cdot$ & . & . & . & . & . & 2 & . & . & . & . & . & . \\
\hline $91 \mathrm{R}-2,38-40$ & 954.28 & & 227 & . & 2 & 8 & . & . & . & 2 & 4 & . & . & . & . & . & 1 & $\cdot$ & 15 & . & . & . & . & . & . & 3 & 2 & . & . & . & . \\
\hline $92 R-1,38.5-40.5$ & 962.485 & & 176 & . & . & . & . & . & . & $\cdot$ & 11 & · & . & . & . & . & . & $\cdot$ & 4 & . & . & 1 & . & . & . & 1 & . & 5 & . & . & . \\
\hline $92 R-2,38-40$ & 963.98 & & 193 & . & $\cdot$ & . & . & . & . & $\cdot$ & 6 & 1 & 1 & . & . & . & . & . & . & 2 & 1 & . & 3 & . & $\cdot$ & . & 2 & . & 1 & . & . \\
\hline $93 R-1,40-41.5$ & 972.1 & & 223 & . & . & 2 & . & . & 1 & 3 & 3 & . & 2 & . & . & . & . & . & . & . & . & . & . & . & . & 3 & 4 & 1 & 2 & . & . \\
\hline $93 R-2,36-37.5$ & 973.56 & IIIE & 276 & . & . & 1 & . & . & $\cdot$ & 10 & 5 & . & 6 & . & . & . & 1 & . & . & . & . & 2 & . & . & . & . & . & . & . & . & \\
\hline $94 R-1,40-41.5$ & 981.7 & & 86 & . & . & 1 & . & . & . & $\cdot$ & 2 & . & . & . & . & . & 1 & . & . & 1 & . & . & . & · & . & . & . & . & 1 & 1 & . \\
\hline $94 R-2,40-41.5$ & 983.2 & & 162 & . & . & 3 & . & . & . & 3 & . & . & 1 & . & . & . & 3 & . & . & . & . & 4 & . & . & . & 3 & . & . & 2 & . & . \\
\hline $94 R-3,35-36.5$ & 984.65 & & 220 & . & . & 3 & . & . & . & . & 7 & . & 10 & . & . & . & . & . & . & . & 3 & . & . & . & . & . & . & . & 2 & . & \\
\hline $95 R-1,42-43.5$ & 991.12 & & 153 & 2 & . & 6 & . & . & . & 2 & · & . & $\cdot$ & . & . & . & . & . & . & . & . & · & . & . & . & . & . & 6 & . & . & \\
\hline $95 R-2,22-23.5$ & 992.42 & & 112 & . & . & . & . & . & . & . & 1 & . & . & . & . & . & . & . & . & . & . & 3 & . & . & . & . & . & 3 & . & . & \\
\hline $96 R-1,40-41.5$ & 1000.8 & & 150 & . & . & . & . & . & 1 & 1 & 8 & . & . & . & . & . & . & . & . & . & . & 1 & . & . & . & . & . & . & . & . & . \\
\hline $97 R-1,40-41.5$ & 1010.4 & & 97 & 1 & . & . & · & . & . & . & . & . & . & . & . & . & . & 1 & . & . & . & . & . & $\cdot$ & . & 1 & $\cdot$ & . & $\cdot$ & $\cdot$ & . \\
\hline $98 R-1,37-38.5$ & 1020.07 & & 184 & . & . & 3 & . & . & 1 & 1 & 4 & . & 3 & 2 & . & . & . & . & . & . & · & $\cdot$ & . & $\cdot$ & · & $\cdot$ & · & $\cdot$ & · & $\cdot$ & . \\
\hline $98 R-2,38.5-40$ & 1021.585 & & 61 & . & . & . & . & . & . & . & $\cdot$ & . & . & . & . & . & . & . & . & . & $\cdot$ & $\cdot$ & . & $\cdot$ & . & $\cdot$ & $\cdot$ & . & . & . & \\
\hline $99 R-1,37-38.5$ & 1029.57 & & 59 & . & . & . & . & . & . & . & 1 & . & . & . & . & . & . & . & . & . & . & 1 & . & . & . & 1 & . & 1 & 1 & . & \\
\hline 100R-1, 33-35 & 1039.13 & & 78 & . & . & . & . & . & . & . & . & . & . & . & . & . & . & . & 1 & . & . & $\cdot$ & . & . & . & $\cdot$ & . & 2 & . & . & 1 \\
\hline $100 R-2,41-42.5$ & 1040.71 & & 12 & & . & . & . & . & . & . & 1 & & . & . & . & . & . & . & . & . & . & . & & . & . & . & . & . & . & . & \\
\hline
\end{tabular}

